\title{
Multiple Equilibria in Noisy Rational Expectations Economies*
}

\author{
Dömötör Pálvölgyi \\ University of Cambridge \\ dp530@dpmms . cam.ac . uk
}

\author{
Gyuri Venter \\ Copenhagen Business School \\ gv.fi@cbs.dk
}

November 7, 2015

\begin{abstract}
This paper studies equilibrium uniqueness in standard noisy rational expectations economies with asymmetric or differential information à la Grossman and Stiglitz (1980) and Hellwig (1980). We show that the standard linear equilibrium of Grossman and Stiglitz (1980) is the unique equilibrium with a continuous price function. However, we also construct a tractable class of equilibria with discontinuous prices that have very different economic implications, including (i) jumps and crashes, (ii) significant revisions in uninformed belief due to small changes in the market price, (iii) "upward-sloping" demand curves, (iv) higher prices leading to future returns that are higher in expectation (price drift) and (v) more positively skewed. Discontinuous equilibria can be arbitrarily close to being fully-revealing. Finally, discontinuous equilibria with the same construction also exist in Hellwig (1980).
\end{abstract}

Keywords: asymmetric information, noisy rational expectations, GrossmanStiglitz, equilibrium multiplicity

*A previous version of this paper was circulated under the title "On the uniqueness of equilibrium in the Grossman-Stiglitz noisy REE model". We thank Dimitri Vayanos, Bradyn Breon-Drish, Christian Hellwig, John Kuong, Aytek Malkhozov, Christine Parlour, Lasse Pedersen, Rémy Praz, Rohit Rahi, Peter Norman Sørensen, Liyan Yang, Haoxiang Zhu, and seminar and conference participants at the Copenhagen Business School, the London School of Economics, the ESSM Minneapolis, the ESSFM Gerzensee, the EWFC St Anton, and the Central Bank Microstructure Conference for helpful comments. The second author gratefully acknowledges financial support from the FRIC Center for Financial Frictions (grant no. DNRF102). 
In their seminal papers, Grossman and Stiglitz (1980) (GS, henceforth) and Hellwig (1980) (H, henceforth) present frameworks for noisy rational expectations economies (REE) which have since become workhorse models studying asymmetric information in competitive financial markets. In such environments, prices have a dual role: to clear the market and to collect and transmit the private information of investors to each other. By introducing noise in the process, the models resolve the paradox of fully-revealing equilibria: Those who expend resources to obtain information achieve better allocation.

Since the information transmission process is noisy, a central question of financial economics is to what extent prices reflect fundamentals versus noise in equilibrium. To answer this question, GS and $\mathrm{H}$ conjecture equilibrium price functions that are linear in the state variables, and show that when random variables are jointly normally distributed and investors have exponential (i.e., CARA) utilities, such equilibria exist and their endogenous parameters are uniquely pinned down. However, the question whether there exist other equilibria of the two models, which are potentially less tractable but offer more realistic predictions, has not been answered.

The first contribution of the paper is to show that the well-known linear equilibrium of the GS model is the unique equilibrium when allowing for any continuous equilibrium price function, linear or not. Our solution method is different from the usual "conjecture and verify" approach, in which conjecturing a specific functional form limits the study of existence and uniqueness to the class of linear functions. As a by-product, we also obtain a more general uniqueness result: Regardless of other distributional assumptions, as long as the demand function of informed traders is additively separable in their signal and the price, which only depends on the distribution of the asset payoff conditional on the signal, there exists at most one equilibrium with a continuous price function.

Our main contribution is to show that once we relax the assumption of a continuous price function, there exist other equilibria. The general idea behind our construction is to partition the state space of the relevant random variables of the model, the signal of informed traders and the noisy supply, into a (potentially infinite) union of disjoint sets, and to create "local noisy REEs" on each of these smaller sets. As long as the images of the local equilibrium price functions are disjoint, a combination of these functions 
becomes a valid equilibrium price function for the overall economy. In our discontinuous equilibria, unlike in the standard equilibrium, uninformed agents not only learn the linear combination of the signal and noisy supply, but also learn from the price level which local set the shocks belong to. In a special case of having two local REEs this means uninformed agents can tell apart if the residual demand they face, i.e., informed demand minus supply, is the result of a good signal coupled with a high supply shock ("liquidity crisis"), or if it is the result of a bad signal and low supply shock ("fundamental crisis"). Hence, uninformed agents know more than what is usually assumed in linear equilibria.

Our leading example of a class of discontinuous but still tractable equilibria, given in closed form, gives rise to a number of phenomena that are absent from the standard linear equilibrium. First, small changes in the asset payoff can lead to large price changes, i.e., jumps and crashes. Second, a small change in the market price can lead to a significant revision in the uninformed belief about expected payoff. Third, the demand curve of uninformed agents is locally downward-sloping but not globally; that is, uninformed agents can demand more at a higher price (in GS it is always downward-sloping). Fourth, uninformed agents' expected return can be higher when the price is higher, leading to price drift (in GS the expected return always decreases in the price, and there is reversal). Fifth, future returns are positively (negatively) skewed after high (low) prices.

Our construction method also opens up a large set of additional equilibria. We further show that discontinuous equilibria with a similar construction and still in closed form can be arbitrarily close to fully revealing if the state space is partitioned sufficiently finely. That is, uninformed agents can learn the signal of informed agents almost perfectly despite the supply shock that was introduced into the GS model to prevent prices to be fully revealing. The Grossman-Stiglitz paradox thus reemerges: If information acquisition is costly, agents do not want to spend resources to become informed, because uninformed traders can almost perfectly extract their signal from market prices.

Finally, we show that our construction method also works in the $\mathrm{H}$ model in which multiple agents with differential information about the payoff trade. This implies that the existence of discontinuous equilibria is not a symptom specific to the two-type setting of GS, but is relevant for a much larger class of informational models. 
The closest papers to ours are Breon-Drish (2010, 2014). Breon-Drish (2010) studies a GS model with normal mixture distributions, finds a continuous equilibrium numerically, and demonstrates that it has very distinct features compared to the usual GS model. Breon-Drish (2014) studies existence and uniqueness among continuous equilibria in GSand H-type models with distributions from the exponential class. Besides establishing uniqueness of the standard linear GS equilibrium among all continuous price functions, our paper complements these studies by constructing discontinuous price functions and showing that they have very different economic implications from the standard GS and $\mathrm{H}$ predictions. Our contribution also lies in the fact that, comparing to these papers, our uniqueness proof is significantly simpler.

Although the theory of fully-revealing REEs is largely complete with many studies on generic existence and uniqueness, and some non-generic examples of non-existence (see, e.g., Radner (1979), Bray (1981), Jordan (1982, 1983)), much less is known about partially-revealing REEs. Previous studies were mainly concerned with the existence of equilibrium; see, for example, Grossman (1976), Grossman and Stiglitz (1980), Hellwig (1980), Diamond and Verrecchia (1981). Ausubel (1990a, 1990b) study existence and uniqueness of a partially-revealing REE under certain conditions; we study uniqueness of equilibrium in the classic models of GS and H. ${ }^{1}$ The idea behind our construction of discontinuous equilibria is closely related to but nevertheless distinct from Jordan (1982). First, Jordan (1982) studies non-noisy environments where the dimension of prices is lower than the dimension of private signals, whereas we study REEs with supply noise. Second, Jordan (1982) shows the existence of equilibria that are discontinuous everywhere (and hence do not describe realistic market behaviour); in contrast, our equilibria are given in closed form, are discontinuous only on zero-measure sets with a countable number of jumps in the price function, and we can study meaningful properties of these equilibria. Finally, unlike Jordan (1982), our construction does not build on deep mathematical results.

There is a related literature that builds on GS and $\mathrm{H}$ to display real-world economic phenomena that the basic models cannot bring forth, by either departing from the

\footnotetext{
${ }^{1}$ See also DeMarzo and Skiadas (1998), who show uniqueness of the fully revealing REE in Grossman (1976) and give examples of partially-revealing equilibria when payoffs are non-normal.
} 
CARA-normal framework or introducing additional frictions. These papers assume, e.g., traders with hedging or portfolio rebalancing motives (see, e.g., Gennotte and Leland (1990)), feedback from prices to fundamentals or production decisions (e.g., Subrahmanyam and Titman (2001), Ozdenoren and Yuan (2008), Sockin and Xiong (2014)), different utility functions and/or distributions of random variables (e.g., Barlevy and Veronesi (2003), Albagli, Hellwig, and Tsyvinski (2013)), trading constraints such as short-sale or borrowing constraints (e.g., Yuan (2005), Bai, Chang, and Wang (2006), Venter (2011)), or higher-order expectations and coordination motives (e.g., Angeletos and Werning (2006)). ${ }^{2}$ In contrast, our focus is on discontinuous equilibria of the basic models, without additional ingredients or different assumptions, and we show that these equilibria already have realistic market properties.

The remainder of the paper is organized as follows. Section 1 presents the textbook GS model. Section 2 shows that the well-known linear equilibrium is the unique continuous equilibrium of the economy. Section 3 provides a class of discontinuous price functions, studies their properties, and provides almost-fully-revealing price functions. Section 4 constructs discontinuous equilibria in the $\mathrm{H}$ model. Finally, Section 5 concludes. Proofs are collected in the Appendix and the Online Appendix.

\section{Model}

This section introduces the baseline asymmetric information model, as in Grossman and Stiglitz (1980). There are two periods, $t=0$ and 1 . Two securities, a riskless and a risky asset, are traded in a competitive market in Period 0, and pay off in Period 1. The riskless asset is in infinite supply, and pays off one unit with certainty. The risky asset is assumed to be in an aggregate supply of $u$ shares, and pays off $d$ units. We assume that $d$ and $u$ are independent, normally distributed random variables, with means normalized to zero and variances $\sigma_{d}^{2}$ and $\sigma_{u}^{2}$, respectively. These distributions constitute a common prior for all agents. We use the riskless asset as numeraire, and denote the price of the risky asset in Period 0 by $p$.

\footnotetext{
${ }^{2}$ See also settings with multiple or derivative assets and cross-market learning, e.g., Admati (1985), Goldstein, Li, and Yang (2014), Chabakauri, Yuan, and Zachariadis (2014) and Malamud (2014).
} 
The asset market is populated by a continuum of agents in measure one. Agents have exponential utility over wealth $W$ in Period $1, U(W)=-\exp (-\alpha W)$, where $\alpha>0$ is the coefficient of absolute risk aversion; to simplify the discussion, we assume that all agents have the same risk-aversion parameter. We normalize agents' initial endowments in the riskless and risky assets to zero, as the assumption of CARA-utility implies that optimal asset holdings are independent of the starting wealth. Thus, if in Period 0 an agent buys $x$ units of the risky asset, her terminal wealth in Period 1 equals capital gains from trading the risky asset: $W=(d-p) x$. None of the agents face any trading (e.g. short-sale or leverage) constraints.

Agents are heterogeneous with respect to their information; they can be either informed or uninformed. Informed traders, in measure $0<\omega<1$, observe a signal $s$ about the risky asset payoff $d$. The signal is given by $s=d+\varepsilon$, where $\varepsilon$ is normal with mean zero and variance $\sigma_{\varepsilon}^{2}$, and is independent of $d$ and $u$. The rest of the agents, in measure $1-\omega$, are uninformed, and do not receive private information about $d$. Besides potentially receiving the signal $s$, agents' information sets are identical; they contain knowledge about the setup of the economy (e.g., the common prior and agents' preferences) and everything agents can infer from the market price $p{ }^{3}$ We denote the expectation and variance conditional on information set $\mathcal{I}$ by $E[. \mid \mathcal{I}]$ and $\operatorname{Var}[. \mid \mathcal{I}]$.

We define an equilibrium of the above economy the standard way:

Definition 1. A rational expectations equilibrium (REE) consists of a measurable price function $P(s, u), P: \mathbb{R}^{2} \rightarrow \mathbb{R}$, and measurable demand functions of informed and uninformed traders, $x_{I}(s, p)$ and $x_{U}(p), x_{I}: \mathbb{R}^{2} \rightarrow \mathbb{R}$ and $x_{U}: \mathbb{R} \rightarrow \mathbb{R}$, such that ${ }^{4}$

1. demand is optimal for informed traders:

$$
x_{I}(s, p) \in \arg \max _{x} E[-\exp \{-\alpha(d-p) x\} \mid s, P(s, u)=p]
$$

\footnotetext{
${ }^{3}$ As it is standard in models with informational asymmetry, the presence of random supply $u$ ensures that the price does not reveal informed traders' signal perfectly, and hence the Grossman-Stiglitz paradox does not apply. Mathematically equivalent alternative ways to introduce noise in the information transmission process would be to assume the presence of noise traders who submit a random priceinelastic demand for reasons exogenous to the model, or, following Wang (1994), to endow informed traders with an investor-specific technology or liquidity shock that correlates with the asset payoff.

${ }^{4}$ Requiring that $P$ is $(s, u)$-measurable implies that the price contains no more information than is possessed by all the investors taken together, and hence satisfies the Kreps (1977) criterion.
} 
2. demand is optimal for uninformed traders:

$$
x_{U}(p) \in \arg \max _{x} E[-\exp \{-\alpha(d-p) x\} \mid P(s, u)=p]
$$

3. the asset market clears at the equilibrium price in all $(s, u)$ states:

$$
\omega x_{I}(s, P(s, u))+(1-\omega) x_{U}(P(s, u))=u .
$$

\section{The unique continuous equilibrium}

The standard solution applied by the literature is the so-called "conjecture and verify" method; see, e.g., Brunnermeier (2001), Vives (2008), or Veldkamp (2011). According to this, solving for an equilibrium of the financial market requires three fairly standard steps: First, we postulate an REE price function $P$. Second, given the price, we derive the belief and optimal demand of uninformed traders. Finally, we check under what conditions the market clears at the conjectured price. The problem with this method is that guessing a particular form for the equilibrium price function naturally limits the set of equilibria available for consideration. Instead, we start by looking at optimal informed demand and the market-clearing condition first, determine what uninformed agents learn through this channel, and show that they cannot learn more if the price function is to be continuous.

Suppose an equilibrium exists, and fix the function $P$. First, we make the observation that informed demand is independent of the equilibrium price function. For an informed trader, who trades with other informed traders endowed with the same information and uninformed traders, the price conveys no additional information relative to observing the signal $s$. Hence, the information set $\mathcal{I}_{I}=\{s, P(s, u)=p\}$ in (1) is equivalent to $\mathcal{I}_{I}=\{s\}$. Given the joint normality of $d$ and $\varepsilon, d$ is normal conditional on $s=d+\varepsilon$ with mean and variance

$$
E[d \mid s]=\beta_{s} s \text { and } \operatorname{Var}[d \mid s]=\beta_{s} \sigma_{\varepsilon}^{2}
$$


where

$$
\beta_{s}=\frac{\operatorname{Cov}[d, s]}{\operatorname{Var}[s]}=\frac{\sigma_{d}^{2}}{\sigma_{d}^{2}+\sigma_{\varepsilon}^{2}}
$$

Conditional normality of $d$ and exponential utility together further imply that (1) is equivalent to a mean-variance problem, and informed traders' optimal demand function is

$$
x_{I}(s, p)=\frac{E[d \mid s]-p}{\alpha \operatorname{Var}[d \mid s]}=\frac{\beta_{s} s-p}{\alpha \beta_{s} \sigma_{\varepsilon}^{2}} .
$$

Next, we substitute this informed demand into the market-clearing condition (3):

$$
\omega \frac{\beta_{s} s-p}{\alpha \beta_{s} \sigma_{\varepsilon}^{2}}+(1-\omega) x_{U}(P(s, u)=p)=u
$$

After rearranging, we obtain

$$
s-C u=g(p)
$$

where $C=\frac{\alpha \sigma_{\varepsilon}^{2}}{\omega}$ and

$$
g(p)=\frac{1}{\beta_{s}} p-(1-\omega) C x_{U}(P(s, u)=p)
$$

Notice that since uninformed agents know the equilibrium form of $P$ and observe $p$, from (8) they know $g(p) .{ }^{5}$ Therefore, from (7), a price realization $p$ always reveals the linear combination $s-C u$, irrespective of what the exact function $P$ is. We summarize and reinterpret this result graphically:

Lemma 1. Suppose an equilibrium exists. Fix a price function $P$ and any realization $p$. Then the set of all possible $(s, u)$ pairs for which $P(s, u)=p$ is a subset of a single straight line on the $(s, u)$ plane with slope $1 / C$.

As such a line can be defined by its intercept with the horizontal axis, we can refer to it either as the line of points that satisfy $s-C u=l$ for a constant $l$, or, with a slight abuse of notation, simply call it line $l$.

The main question is whether a realization $p$ can tell more about $s$ than just revealing $s-C u$. In what follows, we make some simple observations based on (7) to argue that

\footnotetext{
${ }^{5}$ This observation is the noisy equivalent of obtaining information from the traded quantity, as in Kreps (1977).
} 


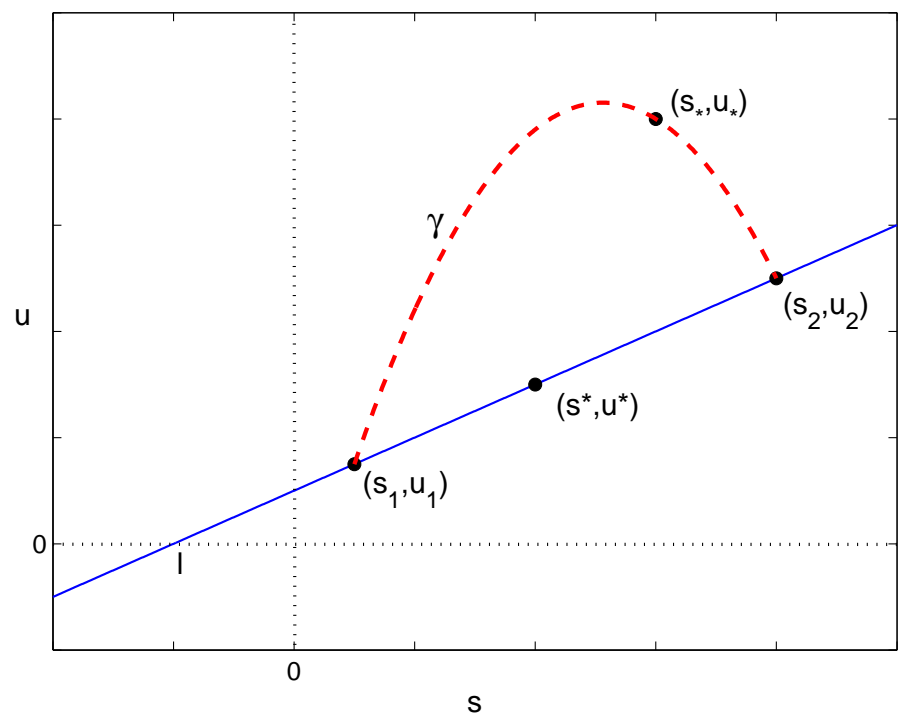

Figure 1. Proof of Lemma 2

If there exist $\left(s_{1}, u_{1}\right)$ and $\left(s_{2}, u_{2}\right)$ such that they satisfy $s-C u=l$ for a fixed constant $l$ but $P\left(s_{1}, u_{1}\right) \neq P\left(s_{2}, u_{2}\right)$, there also exist two points, one on the $l$ line and one outside, on an arbitrary $\gamma$ curve, such that $P\left(s^{*}, u^{*}\right)=P\left(s_{*}, u_{*}\right)$, which contradicts that they should be on a single line with slope $1 / C$.

if $P(s, u)$ is continuous in both arguments, it cannot. Hence, in any equilibrium $p$ and $s-C u$ are observationally equivalent.

Suppose that the converse is true, and $P(s, u)$ is a continuous function of $s$ and $u$ not only through $s-C u$, i.e., it is not $(s-C u)$-measurable. Put graphically, this is equivalent to having two price realizations $p_{1} \neq p_{2}$ so that the information they reveal are disjoint subsets of the same straight line: $g\left(p_{1}\right)=g\left(p_{2}\right)=l$ for some $l$. That is, there are two pairs $\left(s_{1}, u_{1}\right) \neq\left(s_{2}, u_{2}\right)$ that correspond to the two different prices, $P\left(s_{1}, u_{1}\right)=p_{1}$ and $P\left(s_{2}, u_{2}\right)=p_{2}$, while $s_{1}-C u_{1}=l=s_{2}-C u_{2} ;$ see Figure 1 .

As $P(s, u)$ is a continuous function of the random variables $s$ and $u$, the Intermediate Value Theorem implies that if we connect $\left(s_{1}, u_{1}\right)$ and $\left(s_{2}, u_{2}\right)$ with any simple curve of the plane, there must be at least one $(s, u)$ point on this curve where $P(s, u)=\frac{p_{1}+p_{2}}{2}$.

We apply this theorem to two curves. The first is simply the segment connecting $\left(s_{1}, u_{1}\right)$ and $\left(s_{2}, u_{2}\right)$, part of line $l$; there exists at least one point, denoted by $\left(s^{*}, u^{*}\right)$, such that $s^{*}-C u^{*}=l$ and $P\left(s^{*}, u^{*}\right)=\frac{p_{1}+p_{2}}{2}$. The second can be any $\gamma$ curve whose 
intersection with the line is only $\left(s_{1}, u_{1}\right)$ and $\left(s_{2}, u_{2}\right)$. This gives at least one point outside $l$, denoted by $\left(s_{*}, u_{*}\right)$, such that $P\left(s_{*}, u_{*}\right)=\frac{p_{1}+p_{2}}{2}$. Given that $\left(s_{*}, u_{*}\right) \notin l$, it must be that $s_{*}-C u_{*} \neq l$. Hence we found two points of the $(s, u)$ plane that admit the same price, $P\left(s^{*}, u^{*}\right)=P\left(s_{*}, u_{*}\right)=\frac{p_{1}+p_{2}}{2}$, but $g\left(\frac{p_{1}+p_{2}}{2}\right)=s_{*}-C u_{*} \neq s^{*}-C u^{*}=g\left(\frac{p_{1}+p_{2}}{2}\right)$. Graphically (see Figure 1$),\left(s^{*}, u^{*}\right)$ and $\left(s_{*}, u_{*}\right)$ should be on a straight line with slope $1 / C$, but they are clearly not, and it contradicts Lemma 1 . To summarize:

Lemma 2. Suppose an equilibrium exists. Fix a continuous equilibrium price function $P$ and any realization $p$. Then the set of all $(s, u)$ pairs for which $P(s, u)=p$ is a whole straight line on the $(s, u)$ plane with slope $1 / C$.

Therefore, it must be that $g: p \mapsto l$ is a one-to-one partial mapping, i.e., a price realization $p$ is equivalent to the realization of $l=s-C u$. Formally, Lemma 2 implies that $P$ is $(s-C u)$-measurable, i.e., $P(s, u)$ is a function of $s$ and $u$ only through $s-C u$.

To determine the equilibrium function $P$, the final step is to use the prior belief of uninformed traders to derive their optimal demand. As their prior about $(d, s, u)$ is jointly normal, Bayesian updating implies $d$ is also normally distributed conditional on $s-C u$. Combining it with the exponential utility, uninformed agents face a CARAnormal optimization problem, and optimal uninformed demand simply becomes

$$
x_{U}(l, p)=\frac{E[d \mid s-C u=l]-p}{\alpha \operatorname{Var}[d \mid s-C u=l]}
$$

where the expectation is linear in $l$ and the variance is constant:

$$
E[d \mid s-C u=l]=\beta_{l} l \text { and } \operatorname{Var}[d \mid s-C u=l]=\beta_{l}\left(\sigma_{\varepsilon}^{2}+C^{2} \sigma_{u}^{2}\right)
$$

with

$$
\beta_{l}=\frac{\operatorname{Cov}[d, s-C u]}{\operatorname{Var}[s-C u]}=\frac{\sigma_{d}^{2}}{\sigma_{d}^{2}+\sigma_{\varepsilon}^{2}+C^{2} \sigma_{u}^{2}} \cdot{ }^{6}
$$

Combining (3), (9), and (10), and using $l=s-C u, p$ is linear in $l$. After some algebra, we obtain the following result:

\footnotetext{
${ }^{6}$ We note that while we define the optimal uninformed demand as $x_{U}(p)$ in (2), in (9) we slightly abuse our notation with the dependence on $l$. The only reason for this is to emphasize the information and substitution effects, as it is commonly done in the literature.
} 
Theorem 1. If we restrict the equilibrium price function to be continuous, there exists a unique equilibrium of the economy. It is linear in the state variables, $P_{G S}(s, u)=$ $B(s-C u)$, with constants $B=\frac{\sigma_{d}^{2}\left(\sigma_{\varepsilon}^{2}+\omega C^{2} \sigma_{u}^{2}\right)}{\sigma_{\varepsilon}^{2}\left(\sigma_{\varepsilon}^{2}+C^{2} \sigma_{u}^{2}\right)+\sigma_{d}^{2}\left(\sigma_{\varepsilon}^{2}+\omega C^{2} \sigma_{u}^{2}\right)}>0$ and $C=\frac{\alpha \sigma_{\varepsilon}^{2}}{\omega}>0$.

While our main focus is on the standard GS setting, we note that Lemmas 1 and 2 imply a more general uniqueness result. In particular, as long as the optimal informed demand is additively separable in signal $s$ and price $p$, which depends only on the distribution of the asset payoff $d$ conditional on the signal $s$, we obtain the form $(7)$. After Lemma 2, this leads to the price function being $(s-C u)$-measurable, pins down the exact functional form of uninformed demand $x_{U}(l, p)$, and implies there is at most one market-clearing price for each realization of the state variables, irrespective of the unconditional distributions of $d$ and $u .^{7}$

\section{Discontinuous price functions}

In this section we show that if the price function $P$ is $(s, u)$-measurable but not continuous, there are more equilibria. We first argue that if an equilibrium exists, it is perfectly pinned down by the information set of uninformed traders. Afterwards, by choosing an appropriate information set, we provide a tractable class of discontinuous equilibria that differ from the GS price everywhere and are discontinuous only on zero-measure sets.

We start by introducing the following definition:

Definition 2. Let $P$ be an equilibrium price function and $p \in \mathbb{R}$ arbitrary. We call a subset $R$ of the $(s, u)$ plane the $p$-level (information) set, if $P(s, u)=p$ for all $(s, u) \in R$ and $P(s, u) \neq p$ for all $(s, u) \notin R$. If a subset $R$ is the $p$-level set for some $p$, we call it $a$ level set under $P$.

Put differently, a level set is the set of $(s, u)$ points that uninformed investors cannot distinguish from each other in equilibrium because $P$ takes the same value on all of

\footnotetext{
${ }^{7}$ Whether an equilibrium exists, depends on the distribution of the asset payoff conditional on the information content of the price, $d \mid s-C u$. Breon-Drish (2014) provides sufficient conditions both for informed demand being additively separable, and for existence of the continuous equilibrium.
} 
them. Hence, level sets, disjoint by definition, are the atoms of a partition of the $(s, u)$ space that describes what uninformed investors learn in equilibrium.

From Definitions 1 and 2 it is clear that every subset $R$ can be the $p$-level set for at most one $p$, regardless of how the price function $P$ behaves on the rest of the plane. ${ }^{8}$ This is because with the priors on $s$ and $u$ and the information $(s, u) \in R$ the optimal uninformed demand function can be calculated and thus is well-defined. In turn, in each state $(s, u) \in R$ we can obtain the only $p$ that satisfies the market-clearing condition for this uninformed and informed demands. In equilibrium it must be that all $(s, u) \in R$ lead to the same $p$, otherwise uninformed agents would be able to distinguish between these states, and $R$ would not be an equilibrium $p$-level set. Thus, if there exists a $p$ for which $R$ is a $p$-level set, it is uniquely determined. ${ }^{9}$

Suppose now that we have a partition of the plane; based on the above, each subset $R$ has a uniquely pinned down $p$ that can be the equilibrium price realization, if any. Thus, the partition and the corresponding set of $p$ values together determine what the function $P$ can be. However, $P$ must also satisfy a consistency requirement: if we obtain the same price realization $p$ for two regions $R_{1}$ and $R_{2}, P$ is not a valid equilibrium price function, because the $p$-level set is $R_{1} \cup R_{2}$ rather than any of them alone. The following lemma summarizes the relationship between $P$ and its level sets:

Lemma 3. For any subset $R$ there is at most one $p$ such that $R$ is the p-level set under some P. Also, for any partition of the $(s, u)$ plane there exists at most one $P$ such that the parts of the partition are the level sets under $P$.

With the help of Definition 2, we can also rephrase the results of Section 2. Lemma 1 states that for any equilibrium price function $P$, continuous or not, each $p$-level set is a subset of a line with slope $1 / C$, and Lemma 2 claims that if the price function is continuous, then the level sets are the whole lines with slope $1 / C$.

To obtain a discontinuous equilibrium price function, we need to provide a partition of the state space whose atoms are strict subsets of lines with slope $1 / C$. One way to do

\footnotetext{
${ }^{8}$ In fact, $R$ stays a $p$-level set even if we change the values of $P$ outside $R$ to anything different from $p$.

${ }^{9}$ These steps are illustrated in Appendix B for a special case, where we provide the constructive proof for Theorem 2.
} 
this is to "cut" each level set of $P_{G S}$ into two half-lines that become the level sets under the new price function by applying an infinite curve that intersects all lines with slope $1 / C$ exactly once. Hence, this curve, which is sufficient to characterize the new partition, can be given as a function of $l$. Formally, we look for a function $\bar{s}: \mathbb{R} \rightarrow \mathbb{R}$ that cuts each line $l$ into a left half-line, given by $l_{-}=\{(s, u): s-C u=l$ and $s<\bar{s}(l)\}$, and a right half-line $l_{+}=\{(s, u): s-C u=l$ and $s \geq \bar{s}(l)\}$, and the level sets of the new price function $P$ become these left and right half-lines. Our main result is that it is possible to construct a class of equilibria by a "linear cut", i.e., when $\bar{s}$ is linear in $l:{ }^{10}$

Theorem 2. There exist a continuum of discontinuous equilibria of the economy created with a linear cut (LC, henceforth) and given in closed form:

$$
P_{L C}(s, u)= \begin{cases}P_{-}(s-C u) & \text { if } s-\omega C u<\frac{D}{\beta_{s}} \\ P_{+}(s-C u) & \text { if } s-\omega C u \geq \frac{D}{\beta_{s}}\end{cases}
$$

with

$$
P_{-}(l)=(1+\rho) B l-\rho D+\zeta \Psi_{\rho}^{-1}(-\lambda(B l-D))
$$

and

$$
P_{+}(l)=(1+\rho) B l-\rho D-\zeta \Psi_{\rho}^{-1}(\lambda(B l-D))
$$

where $D \in \mathbb{R}$ arbitrary; $B, C$, and $\beta_{s}$ are those in Theorem 1 and (4); $\rho, \zeta$, and $\lambda$ are positive constants given in (A-21); and if $\phi($.$) denotes the pdf of the standard normal$ distribution and $\Phi($.$) is the corresponding cdf, then$

$$
\Psi_{\rho}(x)=(1+\rho) x+\frac{\phi(x)}{\Phi(x)}
$$

is an invertible function whose properties we collect in Appendix A. The functions $P_{-}$ and $P_{+}$are both increasing, infinitely differentiable, and their images are $(-\infty, D)$ and $(D, \infty)$, respectively. $P_{L C}$ takes all real values except for $D$.

\footnotetext{
${ }^{10}$ Graphically, this cut can be thought of as the $(\bar{s}(l), \bar{u}(l))$ points of the $(s, u)$ plane, where $\bar{u}(l)=$ $\frac{\bar{s}(l)-l}{C}$. If $\bar{s}$ is linear in $l, \bar{u}$ is linear too, and the set $\{(\bar{s}(l), \bar{u}(l)): l \in \mathbb{R}\}$ is a straight line on the plane; see the bottom right panel of Figure 2 for illustration. From here $s<\bar{s}(l)$ or $s \geq \bar{s}(l)$ are equivalent to a linear combination of $s$ and $u$ being above or below a threshold, leading to (11). E.g., $s-\omega C u<\frac{D}{\beta_{s}}$ can also be written as $s<\bar{s}(l)$ with $\bar{s}(l)=\frac{D-\omega \beta_{s} l}{(1-\omega) \beta_{s}}$, and we use the two notations interchangeably.
} 
Appendix B contains the details of the constructive proof. It includes four steps. First, we conjecture that the level sets are half-lines. This means a price reveals both the line $l$, i.e., the linear combination of signal $s$ and noisy supply $u$, and that $s$ is above or below a threshold $\bar{s}(l)$, and we formally express the belief of uninformed agents. Second, given the belief, we solve uninformed agents' optimization problem and derive the demand function. Third, we apply market clearing to obtain an implicit equation that the equilibrium price must satisfy. Finally, we show that under the linear cut given in (11) the beliefs are rational, i.e., the level sets are the conjectured half-lines.

Figure 2 illustrates the price function proposed in Theorem 2 and compares it to the GS price of Theorem 1. The upper left panel shows $P_{G S}$ as a function of $s$ and $u$, and the middle left panel shows $P_{G S}$ as a function of $l=s-C u$. The price function is represented by a plane and a line, respectively, because $P_{G S}$ is linear in $l$ and hence in $s$ and $u$. The bottom left panel illustrates the level sets of $P_{G S}$ for different price realizations: e.g., for any $p_{*} \in \mathbb{R}$, the set of points that solve $P_{G S}(s, u)=p_{*}$ is a whole line with slope $1 / C$, represented by asterisks in the bottom left panel.

The upper right panel shows the price function $P_{L C}$ obtained by a linear cut as a function of $s$ and $u$, and the middle right panel plots $P_{L C}$ for values of $l=s-C u$. While $P_{L C}$ is a monotonic function of $s$ and $u$, it is not $l$-measurable any more, as it also depends on whether $s$ is on the right or left half-line of $l$. The dashed curve on the middle right panel corresponds to $P_{-}$, the restriction of $P_{L C}$ to left half-lines; i.e., $P_{-}(l)=P_{L C}(s, u)$ for all $(s, u) \in l_{-}$. Similarly $P_{+}(l)$ tells us what the equilibrium price is on the right half of line $l$, and is represented by the solid curve. For comparison, the dotted line shows $P_{G S}(l)$.

The bottom right panel illustrates the level sets of $P_{L C}$. For any $p \neq D$, the $p$-level set is a half-line with slope $1 / C$; in particular, prices below $D$ indicate a left half-line, and prices above $D$ indicate a right half-line. For instance, as the middle panel shows, prices $p_{\circ}<D<p_{\triangle}$ reveal the same $l$, but they also indicate whether the signal is low or high. Thus, the two level sets, illustrated by circles and by triangles on the bottom right panel, respectively, are the two halves of the same line. The dotted-dashed line indicates the cut $\bar{s}(l)$. Figure 2 shows that it is possible to choose the cut such that 

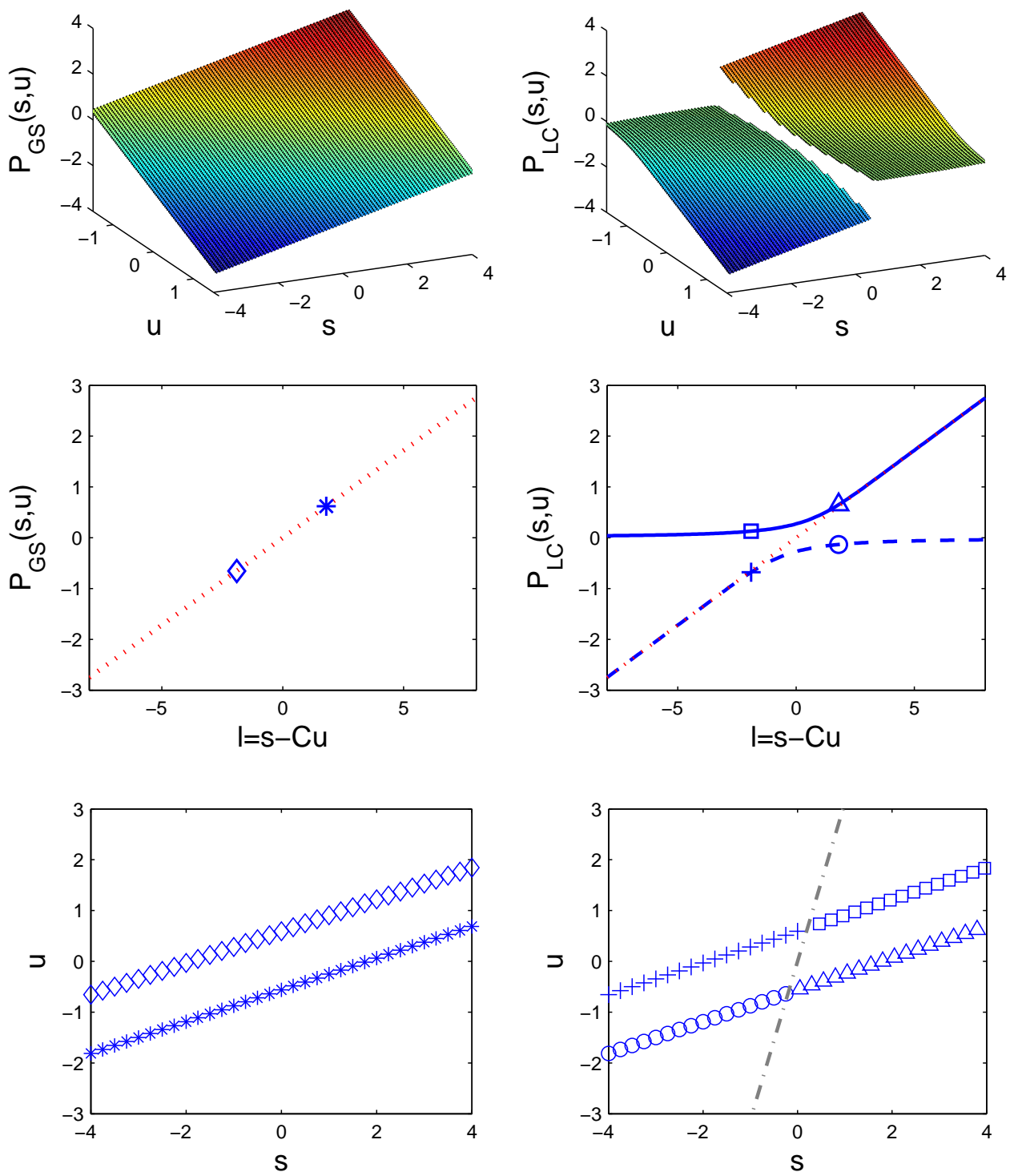

Figure 2. The price function with linear cut, $P_{L C}$

This figure illustrates the price function proposed in Theorem 2 and compares it to the GS price of Theorem 1. The upper left panel shows $P_{G S}$ as a function of $s$ and $u$. The middle left panel shows $P_{G S}$ as a function of $l$, and for two particular $p$ (and $l$ ) realizations the bottom left panel illustrates the level sets of $P_{G S}$ with the same markers. The upper right panel shows the price function $P_{L C}$ obtained by a linear cut as a function of $s$ and $u$. The middle right panel shows $P_{L C}$ for each $l$ : The solid (dashed) curve is $P_{+}$ $\left(P_{-}\right)$as a function of $l$, but $P_{L C}$ is not $l$-measurable; moreover, $D$ is not attained as a price realization. The bottom right panel illustrates the level sets for four different price realizations, with the actual prices plotted on the middle panel with the same markers. The dotted-dashed line indicates the cut $\bar{s}(l)$. The parameters are set to $\sigma_{d}=0.6$, $\sigma_{u}=0.3, \sigma_{\varepsilon}=0.4, \alpha=2, \omega=0.1, D=0$. 
$P_{-}(l)$ and $P_{+}(l)$ are both invertible functions and their images are disjoint, therefore every $p$-level set is exactly one half-line, and $P_{L C}$ is a valid equilibrium price function.

One economic interpretation of the difference between the GS and LC equilibria can be illustrated by revisiting the market-clearing condition (6). When uninformed traders learn the linear combination $l=s-C u$, they obtain information about the residual demand they face, i.e., informed demand minus supply. In the GS equilibrium uninformed agents only know $l$, and they cannot distinguish whether the residual demand is the result of a good signal (informed traders buying more) coupled with a high supply shock, which we think of as a state when asset fundamentals are good but there is a "liquidity crisis", or if it is the outcome of a bad signal and low supply shock, i.e., a "fundamental crisis". In contrast, in the LC equilibrium uninformed traders know the residual demand and also learn about its composition: they are able to tell apart bad liquidity from bad fundamental states. Therefore, in the former they buy more and drive the equilibrium price above the GS price of the same state, and in the latter they buy less and the equilibrium price is below the GS price.

The cut $\bar{s}(l)$ has an important role in this equilibrium. Suppose that a $D$-level set, $D \in \mathbb{R}$, consists of only one point, i.e., is a singleton $(\bar{s}, \bar{u})$. Then $D$ perfectly reveals the informed signal $\bar{s}$, and uninformed traders become informed, too; from (5), the demand of each rational agent is hence $\frac{\beta_{s} \bar{s}-D}{\alpha \beta_{s} \sigma_{\varepsilon}^{2}}$. By market clearing this demand must equal supply $\bar{u}$, and rearranging, we obtain $\bar{s}-\omega C \bar{u}=D / \beta_{s}$. Thus, $\left\{(\bar{s}, \bar{u}) \in \mathbb{R}^{2}\right.$ : $\left.\bar{s}-\omega C \bar{u}=D / \beta_{s}\right\}$ are the points that would individually be $D$-level singletons. ${ }^{11}$ From here the equilibrium price on any left half-line must be below $D$ : the random variable $\left.d\right|_{s=\bar{s}}$ first-order stochastically dominates $\left.d\right|_{s<\bar{s}}$, hence investors demand more and the equilibrium price is higher in the former case. That is, $P_{L C}(s, u)=P_{-}(s-C u)<D$ for all $(s, u)$ satisfying $s-\omega C u<D / \beta_{s}$. Similarly, $P_{L C}(s, u)=P_{+}(s-C u)>D$ for all $(s, u)$ satisfying $s-\omega C u>D / \beta_{s}$.

To illustrate that LC equilibria are not only well-crafted pathological examples of discontinuous equilibria, we next show that the proposed price function has many realistic properties that the standard linear equilibrium does not have.

\footnotetext{
${ }^{11}$ Note that while each of these points is a $D$-level set, their union is not: it would violate Lemma 1.
} 
Proposition 1. Small changes in $s$ and u can lead to large discontinuous changes in the price, i.e., jumps and crashes, given by $\Delta P(l)=P_{+}(l)-P_{-}(l) . \Delta P(l)$ is positive for all $l$, reaches its minimum at $l=D / B$, and has limits $\lim _{l \rightarrow \pm \infty} \Delta P(l)=\infty$.

Proposition 1 describes price sensitivity to signal and supply shocks. We note that large price movements can happen independently of the asset value: since $s-\omega C u=$ $D / \beta_{s}$ is an infinite line on the plane, for all $s \in \mathbb{R}$ there exists a unique $u \in \mathbb{R}$ such that a discontinuous shift occurs at $(s, u)$. These price movements can be arbitrarily large, and larger movements happen at more extreme values of $s$ and $u$, i.e., when $l$ is further away from $D / B$. Interestingly, the discontinuous price movements occur to/from moderate prices close to $D$; see Figure 2.

Proposition 2. Uninformed agents' expectation of the asset payoff is non-monotonic in the price, conditional variance is non-constant, and skewness is non-zero. Moreover, a small change in the market price can lead to a large revision in the expectated payoff.

Figure 3 illustrates the properties of the price function with linear cut compared to the GS price. The upper left panel shows the expected payoff conditional on the price realization, $E[d \mid P=p]$, in the two equilibria. Notably, the conditional expectation is non-monotonic in the LC equilibrium: a higher price realization does not necessarily indicate a higher expected payoff. To understand the particular shape, consider, e.g., the case when the price decreases from $\infty$ to $D$. In this case every price level reveals both the residual demand, $l$, and that both the signal and the supply shock are high $(s \geq \bar{s})$. For very high prices this additional information is not very helpful, because $\bar{s}$ is low. When $p$ decreases, the residual demand $l$ decreases. On the other hand, $\bar{s}(l)$ is a decreasing function of $l$, so $\bar{s}$ increases, and uninformed agents become more certain that informed traders have a good signal. That is, even though residual demand goes down, it decreases because the supply shock increases faster than how informed traders are buying. Nevertheless, signal $s$ increases on average, and $E[d \mid P=p]$ goes to infinity.

The upper right panel shows the conditional variance, $\operatorname{Var}[d \mid P=p]$, in the two equilibria. As the joint distribution of the price and payoff is non-normal, conditional variance is non-constant in the new equilibrium: It is always lower than in the GS 

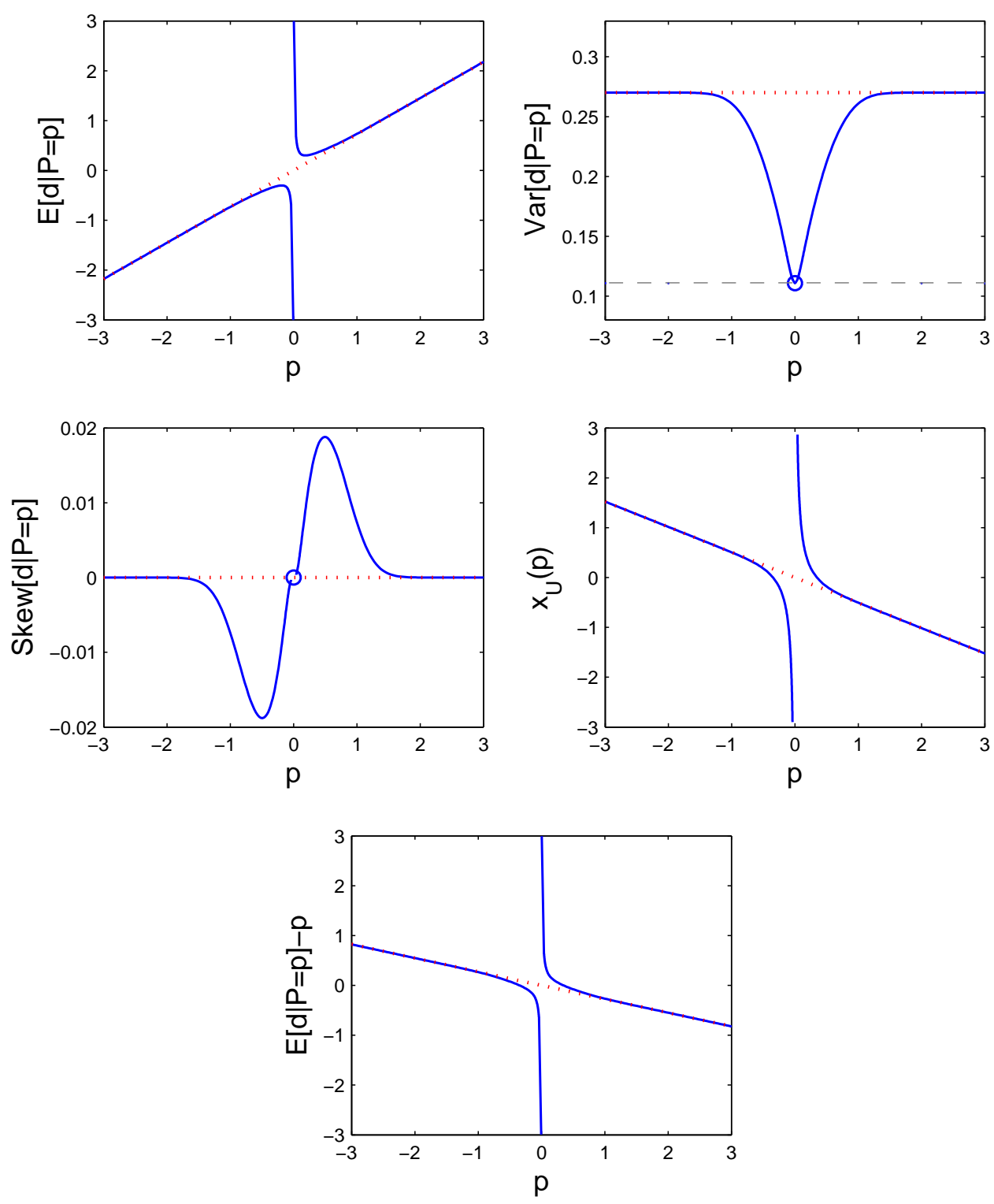

Figure 3. Properties of $P_{L C}$

This figure illustrates the equilibrium properties of the LC price function (solid line on all panels) compared to the GS equilibrium (dotted line). The upper left panel shows that uninformed agents' conditional expectation of the payoff, $E[d \mid P=p]$, is non-monotonic under $P_{L C}$. The upper right panel shows that the conditional variance, $\operatorname{Var}[d \mid P=p]$, is non-constant. The middle left panel shows that the conditional skewness, Skew $[d \mid P=p]$, is positive for high $(p>D)$ prices and negative for low $(p<D)$ prices. The middle right panel shows that the optimal uninformed demand curve, $x_{U}(p)$, is globally not downward-sloping in the LC equilibrium, and a higher price can induce uninformed traders to buy more of the asset. The bottom panel shows the expected return of uninformed traders, $E[d-p \mid P=p]$; a higher price can imply a higher expected return. The parameters are set to $\sigma_{d}=0.6, \sigma_{u}=0.3, \sigma_{\varepsilon}=0.4, \alpha=2, \omega=0.1, D=0$. 
equilibrium, because uninformed investors do not only learn the residual demand, as in GS, but also how informed agents are trading. When the price and thus $l$ are very high, $\bar{s}$ is low, uninformed agents' belief about $s$ is similar to that in the GS equilibrium, and the conditional variance is close to $\operatorname{Var}[d \mid l]$. When $p$ and $l$ decrease, $\bar{s}$ increases, and uninformed agents become more certain that informed traders have a good signal. Payoff volatility is especially small when the price is close to $D$ : uninformed agents' conditional distribution is concentrated on the point $\bar{s}$, and the conditional variance converges to the uncertainty of informed traders, $\operatorname{Var}[d \mid s]$.

The middle left panel shows the conditional skewness of the payoff, Skew $[d \mid P=p]$, in the two equilibria. Unlike in GS, in which joint normality implies zero skewness, it is generally non-zero in the LC equilibrium due to non-normality. In particular, skewness is positive for high prices and negative for low prices. The reason for this is that high prices imply an uninformed belief that is truncated normal with a truncation from below $(s \geq \bar{s})$ and low prices imply beliefs truncated from above $(s<\bar{s})$.

Proposition 3. Non-monotonic belief moments about the payoff lead to an uninformed demand curve $x_{U}(p)$ that is globally not downward-sloping.

The middle right panel of Figure 3 shows the equilibrium demand function of uninformed traders in the two equilibria. In the GS equilibrium, demand is always downward sloping: a higher price induces uninformed agents to buy more because it indicates a higher payoff (information effect), but agents want to buy less due to the substitution effect. As it is well-known, in the GS equilibrium the latter effect dominates the former, and uninformed demand curves slope down. On the other hand, the demand curve in the LC equilibrium is globally non-monotonic even if it is locally downward-sloping everywhere: Uninformed traders are willing to buy more when the price increases from below $D$ to above $D$, because prices above $D$ reveal a high signal with little uncertainty, whereas prices below $D$ reveal a low signal (see the two upper panels of Figure 3 and the corresponding discussion after Proposition 2). That is, the information effect is (locally) so strong in this case that it dominates the substitution effect. 
There is an extensive literature recognizing that if trading in financial markets aggregates information and the resulting prices can feed back to real activity, it introduces complementarities among traders and can lead to upward-sloping demand curves. ${ }^{12}$ In contrast, our LC equilibrium emerges in the standard GS model, and is purely the result of complementarity in trading among uninformed traders who do not possess any private signals, but through their trading affect to what extent the signal of informed traders gets incorporated into the price. That is, if an uninformed trader believes that some prices contain more positive information about the payoff than others, she is willing to buy more of the asset. As all uninformed traders increase their demands, the equilibrium price is pushed above $D$. Thus, high prices indeed reveal better signals than low prices, and expectations are rational.

To further illustrate the differences of the two equilibria, we present two results on return predictability in the LC price function that cannot happen in the standard GS equilibrium. For this end, we study the properties of $d-p$, the future return that investors earn between Periods 0 and 1, conditional on the Period- 0 price $p$, and interpret a higher $p$ as a higher past return. ${ }^{13}$

Our first result is related to the literature on price momentum, documented both in the cross section (recent winners outperforming recent losers; see, e.g., Jegadeesh and Titman (1993)) and in the time series (positive predictability from a security's own past returns; Moskowitz, Ooi, and Pedersen (2012)).

Proposition 4. A higher past return can lead to a higher expected future return, that is, $E[d-p \mid p]$ can be increasing in $p$.

The bottom panel of Figure 3 plots the expected future return conditional on the past return realization in the GS and the LC equilibria. As it is also shown by Banerjee,

\footnotetext{
${ }^{12}$ See, e.g., Subrahmanyam and Titman (2001), Ozdenoren and Yuan (2008), and Sockin and Xiong (2014).

${ }^{13}$ Formally, we could assume that there is a Period -1 before informed agents receive their signal. Since all agents are ex ante identical and have prior $E[d]=0$, following Banerjee, Kaniel, and Kremer (2009) we could assume that if agents traded in this Period $-1, p_{-1}=0$ is the price that would prevail. Thus, $p-p_{-1}=p$ also gives the (past) return between Periods -1 and 0 . A proper treatment of a Period -1 would be to assume rational agents trading and optimizing in a 3-period (dynamic) setting, as in, e.g., Vayanos and Wang (2012), and to obtain an equilibrium $p_{-1}$. Due to invariance to additive constants, however, the results of Propositions 4 and 5 would not change.
} 
Kaniel, and Kremer (2009), in the GS equilibrium expected return is always downwardsloping, higher prices imply lower subsequent expected returns, and the equilibrium displays price reversal. Notably, however, the expected return is non-monotonic in the new equilibrium: a higher price realization can indicate a higher expected return when the price is close to $D$, because a price slightly above $D$ reveals a high signal-high supply shock combination, leading to a jump in the payoff expectation of uninformed traders. Thus, in the LC equilibrium higher prices (higher past returns) can imply higher subsequent returns, and the equilibrium can display price drift. ${ }^{14}$

Several recent empirical papers study the asset pricing implications of skewness, and find that securities with positively skewed returns tend to be overpriced; see, e.g., Boyer, Mitton, and Vorkink (2010) and Conrad, Dittmar, and Ghysels (2013). Previous theories in line with these predictions generally obtain non-trivial return skewness by exogenously assuming non-normal asset payoff distributions. ${ }^{15}$ In contrast, in our model non-zero skewness is due to a non-linear equilibrium price function of normally distributed state variables in the standard GS framework:

Proposition 5. Conditional skewness of future returns, Skew $[d-p \mid p]$, is negative for low prices and positive for high prices.

Because skewness is invariant to additive constants, Skew $[d-p \mid p]=$ Skew $[d \mid p]$, this proposition formalizes the middle left panel of Figure 3. As discussed in Proposition 2, beyond the residual demand, high prices also reveal that the signal is above a threshold $(s \geq \bar{s})$, which means that future returns are bounded from below but can be arbitrarily

\footnotetext{
${ }^{14}$ With the help of the expected return, we can revisit the uninformed demand curve and show that while its overall shape is different from that in the GS equilibrium, it can be motivated in a similar way. In the linear equilibrium uninformed demand is given by $\frac{E\left[d \mid P_{G S}=p\right]-p}{\alpha \operatorname{Var}\left[d \mid P_{G S}=p\right]}$, and since the variance is constant and the expected return, defined as $E\left[d \mid P_{G S}=p\right]-p$, decreases linearly in the price, uninformed demand also decreases linearly. The bottom panel of Figure 3 illustrates the expected return in the LC equilibrium. Combining it with the shape of the conditional variance, $x_{U}(p)$ is very similar to what $\frac{E\left[d \mid P_{L C}=p\right]-p}{\alpha \operatorname{Var}\left[d \mid P_{L C}=p\right]}$ would be. That is, uninformed demand in the discontinuous equilibrium behaves approximately the same way as the optimal demand in a mean-variance world, even though it is not a CARA-normal setting.

${ }^{15}$ See, e.g., Brunnermeier and Parker (2005) and Brunnermeier, Gollier, and Parker (2007), who develop models of optimal (non-rational) expectations; Barberis and Huang (2008), who use cumulative prospect theory; Mitton and Vorkink (2007), who set up a model where investors have heterogeneous preference for skewness; and Albagli, Hellwig, and Tsyvinski (2013), who provide a general theory of information aggregation that can be applied outside the standard CARA-normal framework.
} 
high, creating positive skewness. Conversely, low prices reveal that the signal is from a lower half-line, i.e., future returns are capped from above but can be arbitrarily negative. In contrast, in the GS equilibrium the price is a linear function of the state variables, and due to joint normality, return skewness is always zero.

\subsection{On the possibility of informationally efficient markets}

So far we have only considered equilibria where we partition the $(s, u)$ plane into two halfplanes by one linear cut $\bar{s}$. The equilibrium presented in Theorem 2 , however, has two important features that allow us to generalize our results: First, the cut $s-\omega C u=D / \beta_{s}$ (or, equivalently, $\bar{s}(l)=\frac{D-\omega \beta_{s} l}{(1-\omega) \beta_{s}}$ ) leads to a partition where prices on left half-lines, $P_{-}$, are all below $D$, whereas prices on right half-lines, $P_{+}$, are all above $D$. Second, the functions $P_{-}$and $P_{+}$obtained with this linear cut are monotonically increasing.

Based on these observations, we next consider a countable number of subsets that we partition the $(s, u)$ plane into. Let us denote $\bar{s}_{n}(l)=\frac{D_{n}-\omega \beta_{s} l}{(1-\omega) \beta_{s}}$ for some indices $n \in \mathcal{Z} \subseteq \mathbb{Z}$, where $D_{n} \in \mathbb{R}$ arbitrary that satisfy $D_{n}<D_{n+1}$ for all $n \in \mathcal{Z}$. We show that these cuts together also generate a valid REE price function:

Theorem 3. There exists an equilibrium of the asset market that is created by a countable number of parallel linear cuts and given by

$$
P_{L C m}(s, u)=\sum_{n \in \mathcal{Z}} 1_{\left\{\frac{D_{n}}{\beta_{s}} \leq s-\omega C u<\frac{D_{n+1}}{\beta_{s}}\right\}} P_{n}(s-C u),
$$

where $1_{\{.\}}$is the indicator function; $P_{n}, n \in \mathcal{Z}$, are monotonically increasing $\mathbb{R} \rightarrow \mathbb{R}$ functions given in closed form in (A-31), and $D_{n}<P_{n}(l)<D_{n+1}$ for all $l \in \mathbb{R}$.

Our construction method opens up a large set of additional equilibria besides the LC equilibrium. $P_{L C m}$ is a simple generalization of $P_{L C}$ by partitioning the state space to a countable number of local REEs instead of just two. $P_{L C m}$ is a union of (potentially) infinitely many local price functions $P_{n}$ that have disjoint domains and disjoint images. Therefore, from a given price realization uninformed traders first learn which subset of the state space $s$ is located in, in the form of a strip $D_{n} / \beta_{s} \leq s-\omega C u<D_{n+1} / \beta_{s}$. 

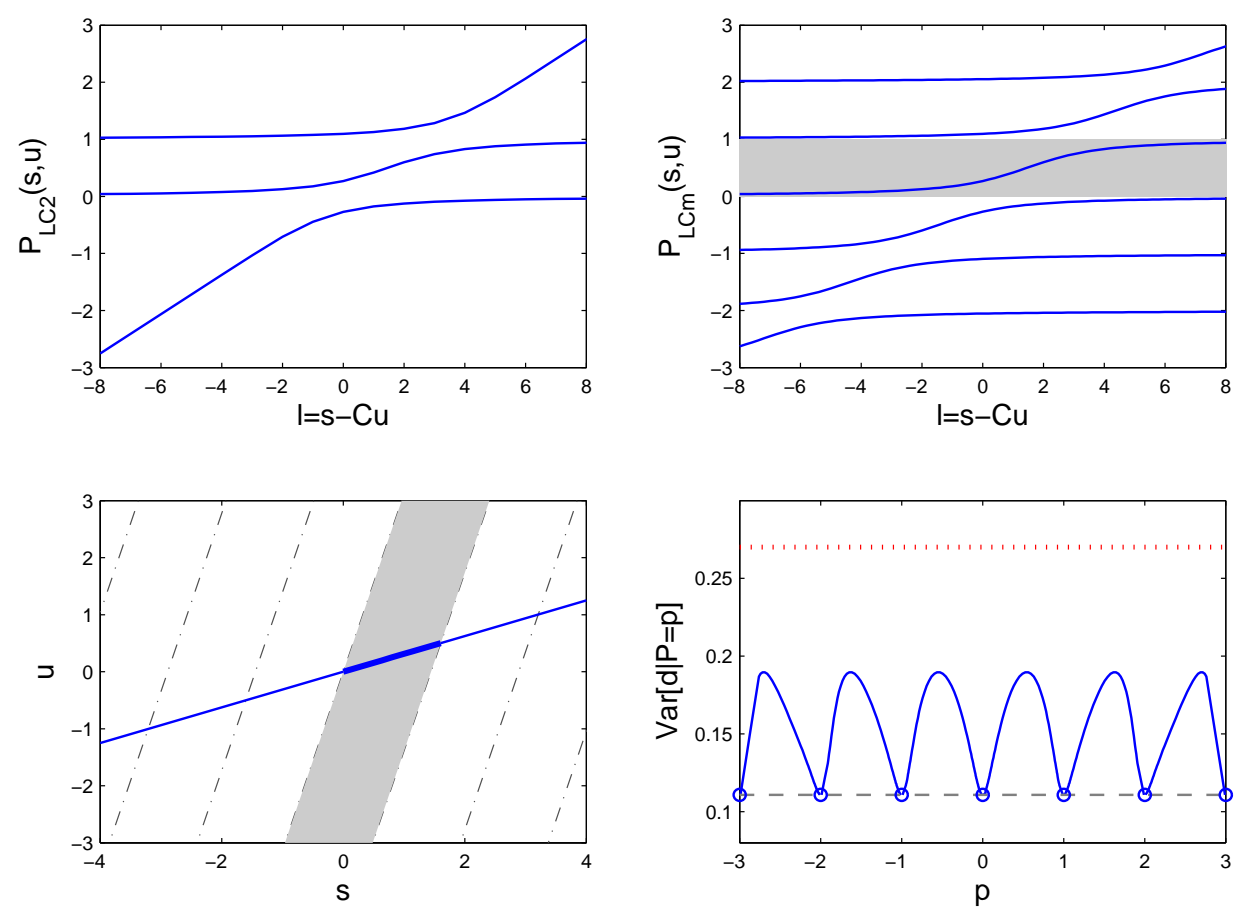

Figure 4. Properties of $P_{L C m}$

The top left panel illustrates the price function $P_{L C 2}$ with only two parallel linear cuts at $D_{0}=0$ and $D_{1}=1$. The top right panel illustrates the price function $P_{L C m}$ created by linear cuts for all $D_{n}=n, n \in \mathbb{Z}$. The bottom left panel shows what uninformed traders learn about $s$ from a price realization $p$ of $P_{L C m}$. If, e.g., $D_{0}=0<p<1=D_{1}$, illustrated by the shaded area of the top right panel figure, uninformed traders learn that $\bar{s}_{0}(l) \leq s<\bar{s}_{1}(l)$, i.e., the signal is from the shaded area of the bottom left panel figure. The price function, through its local component (in this case $P_{0}$ ), also reveals $s-C u$, i.e., $s$ is on the solid line of the bottom left panel. The two observations together reveal that $s$ is from the intersection of the shaded area and the solid line, i.e., the bold solid line. The bottom right panel shows the uncertainty faced by uninformed agents as a function of the price, $\operatorname{Var}\left[d \mid P_{L C m}=p\right]$ (solid line), together with the conditional variance of uninformed agents in the GS equilibrium, $\operatorname{Var}[d \mid l]$ (dotted line), and the conditional variance of informed agents, $\operatorname{Var}[d \mid s]$ (dashed line). The other parameters are set to $\sigma_{d}=0.6, \sigma_{u}=0.3, \sigma_{\varepsilon}=0.4, \alpha=2, \omega=0.1$.

Theorem 3 also states that all $P_{n}$ functions are increasing, thus invertible, and uninformed agents learn the linear combination $l=s-C u$. Hence, overall they learn that the signal is from the segment $\left[\bar{s}_{n}(l), \bar{s}_{n+1}(l)\right)$. The top left panel of Figure 4 shows an equilibrium price function (denoted by $P_{L C 2}$ ) with only two cuts at $D_{0}$ and $D_{1}>D_{0}$. The top right panel of Figure 4 illustrates $P_{L C m}$ for infinitely many cuts for different 
values of $l$, and the bottom left panel shows what uninformed traders learn about $s$ from a price realization $p$.

Next we define $\Delta s=\sup _{n, l}\left(\bar{s}_{n+1}(l)-\bar{s}_{n}(l)\right)=\sup _{n} \frac{D_{n+1}-D_{n}}{(1-\omega) \beta_{s}} . \Delta s$ measures how fine the partition is, i.e., how close cuts are to each other. Since uninformed agents learn from a price realization the interval in which $s$ is, the smaller $\bar{s}_{n+1}-\bar{s}_{n}$ becomes (while being non-negative by definition), the more precisely uninformed agents can infer $s$ from the market price. Another way to illustrate the precision of uninformed agents' information is to consider the conditional variance $\operatorname{Var}[d \mid P=p]$, which is simply $\operatorname{Var}\left[d \mid \bar{s}_{n}(l) \leq s<\bar{s}_{n+1}(l)\right]$ when $D_{n}<p=p(l)<D_{n+1}$. We define the degree of uncertainty uninformed agents face in equilibrium, in addition to that faced by informed traders, by $\Delta \operatorname{Var}=\sup _{p} \operatorname{Var}[d \mid P=p]-\operatorname{Var}[d \mid s]$; see the bottom right panel of Figure 4. When $D_{n+1}-D_{n} \rightarrow 0$, uninformed traders learn the signal $s$ almost perfectly, and the difference in uncertainty disappears. ${ }^{16,17}$ Formally, we have the following result:

Theorem 4. For every $\epsilon>0$ there exist equilibria of the asset market such that the partition of the state space is finer than $\epsilon$, i.e., $\Delta s<\epsilon$. Moreover, for every $\delta>0$ there exist equilibria such that uninformed traders' conditional variance about the asset payoff is closer to the conditional variance of informed traders than $\delta$, i.e., $\Delta V a r<\delta$.

Theorem 4 states that discontinuous equilibria, created by infinitely many parallel linear cuts, can be arbitrarily close to fully-revealing by partitioning the state space sufficiently finely. In a series of equilibria that satisfy $\Delta s \rightarrow 0$ or $\Delta V$ ar $\rightarrow 0$ uninformed agents can learn the signal of informed agents almost perfectly. This leads to almostfull revelation despite the supply shock $u$ that is introduced to the GS model to prevent prices from being fully-revealing, and in spite of the fact that a fully-revealing REE of the economy does not exist. The Grossman-Stiglitz paradox thus reemerges: If information acquisition is costly, agents do not want to become informed, because uninformed traders can extract their information almost perfectly from market prices for free.

\footnotetext{
${ }^{16}$ Notice that, unlike in the majority of the literature, in all equilibria discussed in Section 3 , the posterior belief of uninformed traders is non-normal, and information precision is not fully captured by variance. Nevertheless, $\operatorname{Var}[d \mid P=p]$ converging to $\operatorname{Var}[d \mid s]$ uniformly is sufficient for uninformed traders to learn the signal almost perfectly.

${ }^{17}$ Alternatively, we could measure informational (in)efficiency by the ratio $\operatorname{Var}[d \mid s] / \operatorname{Var}[d \mid p]$, as in GS. Theorem 4 would then state that there exist equilibria with this ratio being arbitrarily close to 1.
} 


\section{Equilibrium multiplicity with differential information}

To show that the existence of discontinuous equilibria is not specific to the GS model, but is relevant for a much larger class of informational models, we next study an economy that generalizes GS to differential information, following Hellwig (1980) and Diamond and Verrecchia (1981), and consider its continuum-agent version in the spirit of Admati (1985). Similarly to the GS model, there are two periods, $t=0$ and 1 . Two securities, a riskless and a risky asset, are traded in a competitive market in Period 0, and pay off in Period 1. The riskless asset is in infinite supply, and pays off one unit with certainty. The risky asset is assumed to be in an aggregate supply of $u$ shares, and pays off $d$ units. The random variables $d$ and $u$ are independent and have jointly normal distribution with zero means and variances $\sigma_{d}^{2}$ and $\sigma_{u}^{2}$, respectively. We use the riskless asset as numeraire, and denote the price of the risky asset in Period 0 by $p$.

The asset market is populated by a continuum of agents in measure one. Agents have exponential utility over wealth $W$ in Period $1, U(W)=-\exp (-\alpha W)$, where $\alpha>0$ is the coefficient of absolute risk aversion. Normalizing agents' initial endowments in the riskless and risky assets to zero, if an agent buys $x$ units of the risky asset, her terminal wealth in Period 1 equals capital gains from trading: $W=(d-p) x$. None of the agents face any trading (e.g., short-sale or leverage) constraints.

Unlike in GS, we assume that all traders receive some additional information about the payoff of the risky asset. In particular, agent $i$ receives private signal $s_{i}=d+\varepsilon_{i}$ where $\varepsilon_{i} \sim N\left(0, \sigma_{\varepsilon}^{2}\right)$ i.i.d across agents and independent of both $d$ and $u$. Throughout our analysis we assume that the law of large numbers holds, namely the "average" of signals $s_{i}$ is $d: \int_{i} s_{i} \mathrm{~d} i=d$. Besides the private signals, agents also make use of the information content of the market price $p .^{18}$

We define an equilibrium of the above economy the standard way:

\footnotetext{
${ }^{18}$ Our result on multiple equilibria in the differential information model does not hinge on the assumption of infinitely many agents. A finite number of traders, however, would further complicate our calculations, because in that version the market price would be a function of the supply noise and the average of private signals, instead of the true payoff, and hence would include two noise components beyond the payoff $d$.
} 
Definition 3. A rational expectations equilibrium (REE) consists of a measurable price function $P(d, u), P: \mathbb{R}^{2} \rightarrow \mathbb{R}$, and measurable individual strategies $x_{i}\left(s_{i}, p\right), x_{i}: \mathbb{R}^{2} \rightarrow$ $\mathbb{R}$, such that

1. demand is optimal for trader $i$ :

$$
x_{i}\left(s_{i}, p\right) \in \arg \max _{x} E\left[-\exp \{-\alpha(d-p) x\} \mid s_{i}, P=p\right]
$$

2. the asset market clears at the equilibrium price in all states:

$$
\int_{i} x_{i}\left(s_{i}, P(d, u)\right) \mathrm{d} i=u
$$

The standard way of solving the model is to conjecture an equilibrium price function (or its information content) linear in $d$ and $u$, deriving optimal demands, plugging them into the market-clearing condition, and finally matching the coefficients with the conjecture. These steps yield the following result:

Theorem 5 (Hellwig (1980), Admati (1985)). There exists a unique linear equilibrium of the economy with the price function given by $P_{H}(d, u)=F(d-G u)$, with constants $F=\frac{\sigma_{d}^{2} \sigma_{\varepsilon}^{2}+\sigma_{d}^{2} G^{2} \sigma_{u}^{2}}{\sigma_{d}^{2} \sigma_{\varepsilon}^{2}+\sigma_{d}^{2} G^{2} \sigma_{u}^{2}+\sigma_{\varepsilon}^{2} G^{2} \sigma_{u}^{2}}>0$ and $G=\alpha \sigma_{\varepsilon}^{2}>0$.

Unfortunately, we have not been able to obtain a uniqueness result on continuous equilibrium functions, as in Section 2. ${ }^{19}$ We nevertheless provide a class of discontinuous (and hence non-linear) equilibria of the model with dispersed information:

Theorem 6. There exist a continuum of discontinuous equilibria given by

$$
P_{L C, H}(d, u)= \begin{cases}P_{-}(l)=J-\chi \Psi_{0}\left(\frac{J-F l}{\chi}\right)=F l-\chi \frac{\phi\left(\frac{J-F l}{\chi}\right)}{\Phi\left(\frac{J-F l}{\chi}\right)} & \text { if } d<J \\ P_{+}(l)=J+\chi \Psi_{0}\left(-\frac{J-F l}{\chi}\right)=F l+\chi \frac{\phi\left(-\frac{J-F l}{\chi}\right)}{\Phi\left(-\frac{J-F l}{\chi}\right)} & \text { if } d \geq J\end{cases}
$$

where $J \in \mathbb{R}$ arbitrary, $F$ and $G$ are those in Theorem $5, l=d-G u, \chi=\sigma_{d} \sqrt{1-F}$, and $\Psi_{0}(x)=x+\frac{\phi(x)}{\Phi(x)}$ is a positive and increasing (hence invertible) function.

\footnotetext{
${ }^{19}$ The reason for this is that informed traders in the GS model do not learn from the price, thus their demand is independent from the price function. In the present setting changing the price function affects optimal demands in non-trivial ways, because all agents can learn new information from prices.
} 
The construction of this equilibrium is the same as in the GS setup; moreover, the properties of the equilibrium are qualitatively identical to those described in Section $3 .^{20}$ Discontinuous equilibria are thus not restricted to the GS model, and can be relevant in a larger class of informational models.

\section{Conclusion}

The standard method of conjecturing and then verifying a linear equilibrium price function has become widely used in models of asymmetric or differential information. While papers following this technique usually show that the price function is unique in the linear class, they do not know anything outside the linear class (see, e.g., Brunnermeier (2001), Vives (2008), and Veldkamp (2011), and the references therein). In this paper we study whether there exist other equilibria, to understand whether predictions based on the linear equilibrium are robust predictions about all the equilibria of such models.

First, using a solution method different from the usual "conjecture and verify" approach, we show that the linear equilibrium of the GS model is unique when allowing for any continuous equilibrium price function. We also construct discontinuous equilibria in the GS and H models and show that they lead to very different (and realistic) economic phenomena compared to the linear one, and that there exist discontinuous equilibria arbitrarily close to being fully-revealing.

Throughout our analysis we restrict our attention to the settings of GS and H. There are many directions we could look to extend our results. First, it would be interesting to see whether we could provide similar statements about equilibrium uniqueness when allowing for a larger class of distributions and utility functions. Second, an important question is whether our (non-)uniqueness results would hold in a modification of the model that incorporates imperfect competition, as in Kyle (1989). These problems are left for future research.

\footnotetext{
${ }^{20}$ For example, quite surprisingly but similar the GS model, the cut is again along the points that would correspond to singleton level sets; see the related discussion on page 15.
} 


\section{References}

Admati, A. R. (1985): "A noisy rational expectations equilibrium for multi-asset securities markets," Econometrica, 53, 629-658.

Albagli, E., C. Hellwig, And A. Tsyvinski (2013): "A theory of asset prices based on heterogeneous information," Working paper, Toulouse School of Economics.

Angeletos, G.-M., And I. Werning (2006): "Crises and prices: information aggregation, multiplicity, and volatility," American Economic Review, 96, 1720-1736.

Ausubel, L. M. (1990a): "Insider trading in a rational expectations economy," American Economic Review, 80, 1022-1041.

(1990b): "Partially-revealing rational expectations equilibrium in a competitive economy," Journal of Economic Theory, 50, 93-126.

Bai, Y., E. C. Chang, And J. WAng (2006): "Asset prices under short-sale constraints," Working paper, MIT.

Banerjee, S., R. Kaniel, and I. Kremer (2009): "Price drift as an outcome of differences in higher order beliefs," Review of Financial Studies, 22, 3797-3734.

Barberis, N., And M. Huang (2008): "Stocks as lotteries: The implications of probability weighting for security prices," American Economic Review, 98, 2066-2100.

BArlevy, G., And P. Veronesi (2003): "Rational panics and stock market crashes," Journal of Economic Theory, 110, 234-263.

Boyer, B., T. Mitton, And K. Vorkink (2010): "Expected idiosyncratic skewness," Review of Financial Studies, 23, 169-202.

BraY, M. (1981): "Futures trading, rational expectations, and the efficient markets hypothesis," Econometrica, 49, 575-596.

Breon-Drish, B. (2010): "Asymmetric information in financial markets: anything goes," Working paper, Stanford University.

(2014): "On existence and uniqueness of equilibrium in a class of noisy rational expectations models," Working paper, Stanford University.

Brunnermeier, M. K. (2001): Asset pricing under asymmetric information. Oxford Univeristy Press, New York, NY.

Brunnermeier, M. K., C. Gollier, and J. Parker (2007): "Optimal beliefs, asset prices and the preference for skewed returns," American Economic Review Papers and Proceedings, 97, 159-165.

Brunnermeier, M. K., And J. Parker (2005): "Optimal expectations," American Economic Review, 95, 1092-1118.

Chabakauri, G., K. Yuan, And K. E. Zachariadis (2014): "Multi-asset noisy rational expectations equilibrium with contingent claims," Working paper, LSE. 
Conrad, J., R. F. Dittmar, and E. Ghysels (2013): "Ex ante skewness and expected stock returns," Journal of Finance, 68, 85-124.

DeMarzo, P., and C. Skiadas (1998): "Aggregation, determinacy, and informational efficiency for a class of economies with asymmetric information," Journal of Economic Theory, $80,123-152$.

Diamond, D. W., AND R. E. VerRecchia (1981): "Information aggregation in a noisy rational expectations economy," Journal of Financial Economics, 9, 221-235.

Gennotte, G., And H. Leland (1990): "Market liquidity, hedging, and crashes," American Economic Review, 80, 999-1021.

Goldstein, I., Y. LI, AND L. YAng (2014): "Speculation and hedging in segmented markets," Review of Financial Studies, 27, 881-922.

Greene, W. H. (2003): Econometric analysis. Prentice Hall, Upper Saddle River, NJ.

Grossman, S. J. (1976): "On the efficiency of competitive stock markets where traders have diverse information," Journal of Finance, 31, 573-585.

Grossman, S. J., AND J. E. Stiglitz (1980): "On the impossibility of informationally efficient markets," American Economic Review, 70, 393-408.

HeLLWIG, M. F. (1980): "On the aggregation of information in competitive markets," Journal of Economic Theory, 22, 477-498.

Jegadeesh, N., and S. Titman (1993): "Returns to buying winners and selling losers: Implications for stock market efficiency," Journal of Finance, 48, 65-91.

JorDAN, J. (1982): "The generic existence of rational expectations equilibrium in the higher dimensional case," Journal of Economic Theory, 26, 224-243.

(1983): "On the efficient market hypothesis," Econometrica, 51, 1325-1344.

Kreps, D. M. (1977): "A note on "fulfilled expectations" equilibria," Journal of Economic Theory, 14, 32-43.

Kyle, A. (1989): "Informed speculation with imperfect competition," Review of Economic Studies, 56, 317-355.

Malamud, S. (2014): "Price discovery through options," Working paper, EPFL.

Mitton, T., And K. Vorkink (2007): "Equilibrium underdiversification and the preference for skewness," Review of Financial Studies, 20, 1255-1288.

Moskowitz, T., Y. H. Ooi, And L. H. Pedersen (2012): "Time series momentum," Journal of Financial Economics, 104, 228-250.

Ozdenoren, E., And K. YuAn (2008): "Feedback effects and asset prices," Journal of Finance, 63, 1939-1975.

RADNER, R. (1979): "Rational expectations equilibrium: generic existence and the information revealed by prices," Econometrica, 47, 655-678. 
SAmpford, M. R. (1953): "Some inequalities on Mills' ratio and related functions," Annals of Mathematical Statistics, 24, 130-132.

Sockin, M., AND W. XIOng (2014): "Informational frictions and commodity markets," Working paper, Princeton University.

Subrahmanyam, A., And S. Titman (2001): "Feedback from stock prices to cash flows," Journal of Finance, 56, 2389-2413.

VAYANOS, D., AND J. WANG (2012): "Liquidity and asset returns under asymmetric information and imperfect competition," Review of Financial Studies, 25, 1339-1365.

VeldKamp, L. (2011): Information choice in macroeconomics and finance. Princeton, Princeton, NJ.

Venter, G. (2011): "Short-sale constraints and credit runs," Working paper, Copenhagen Business School.

VIVES, X. (2008): Information and learning in markets: The impact of market microstructure. Princeton, Princeton, NJ.

WANG, J. (1994): "A model of competitive stock trading volume," Journal of Political Economy, 102, 127-167.

YUAN, K. (2005): "Asymmetric price movements and borrowing constraints: A rational expectations equilibrium model of crises, contagion, and confusion," Journal of Finance, 60, $247-292$.

\section{Appendix A Preliminary results}

In this appendix we collect properties of some functions that are used in Appendix B. The functions $\phi($.$) and \Phi($.$) denote the pdf and cdf of the standard normal distribution, respectvely.$ The proofs of these lemmas are provided in the Online Appendix.

Lemma 4. The function

$$
\Psi_{-1}(x)=\frac{\phi(x)}{\Phi(x)}
$$

has the following three sets of properties:

(i) $\Psi_{-1}(x)>0 \forall x \in \mathbb{R}$, and its limits are $\lim _{x \rightarrow-\infty} \Psi_{-1}(x)=\infty$ and $\lim _{x \rightarrow \infty} \Psi_{-1}(x)=0$.

(ii) $\Psi_{-1}(x)$ is decreasing; its slope satisfies $-1<\Psi_{-1}^{\prime}(x)<0$ for all $x \in \mathbb{R}$ with limits $\lim _{x \rightarrow-\infty} \Psi_{-1}^{\prime}(x)=-1$ and $\lim _{x \rightarrow \infty} \Psi_{-1}^{\prime}(x)=0$, and $\Psi_{-1}^{\prime}$ increases monotonically in between.

(iii) $\Psi_{-1}(x)$ is convex: $\Psi_{-1}^{\prime \prime}(x)>0$ for all $x \in \mathbb{R}$.

Lemma 5. Suppose $\rho \geq 0$ constant. The function

$$
\Psi_{\rho}(x)=(1+\rho) x+\frac{\phi(x)}{\Phi(x)}
$$

has the following properties: 
(i) If $\rho=0, \Psi_{0}(x)>0$ for all $x \in \mathbb{R}$, and its limits are $\lim _{x \rightarrow-\infty} \Psi_{0}(x)=0$ and $\lim _{x \rightarrow \infty} \Psi_{0}(x)=\infty$. If $\rho>0, \Psi_{\rho}(x)$ takes every real value and its limits are $\lim _{x \rightarrow-\infty} \Psi_{\rho}(x)=$ $-\infty$ and $\lim _{x \rightarrow \infty} \Psi_{\rho}(x)=\infty$.

(ii) $\Psi_{\rho}(x)$ is increasing; its slope satisfies $\rho<\Psi_{\rho}^{\prime}(x)<1+\rho$ for all $x \in \mathbb{R}$ with limits $\lim _{x \rightarrow-\infty} \Psi_{\rho}^{\prime}(x)=\rho$ and $\lim _{x \rightarrow \infty} \Psi_{\rho}^{\prime}(x)=1+\rho$, and $\Psi_{\rho}^{\prime}$ increases monotonically in between.

(iii) $\Psi_{\rho}(x)$ is convex: $\Psi_{\rho}^{\prime \prime}(x)>0$ for all $x \in \mathbb{R}$.

Lemma 6. Suppose $\rho \geq 0$ and $M>0$ constants. The function

$$
\Omega_{\rho}(x)=(1+\rho) x+\frac{\phi(x+M)-\phi(x-M)}{\Phi(x+M)-\Phi(x-M)}
$$

has the following properties:

(i) If $\rho=0, \Omega_{0}$ has limits $\lim _{x \rightarrow-\infty} \Omega_{0}(x)=-M$ and $\lim _{x \rightarrow \infty} \Omega_{0}(x)=-M$. If $\rho>0$, $\Omega_{\rho}(x)$ takes every real value and its limits are $\lim _{x \rightarrow-\infty} \Omega_{\rho}(x)=-\infty$ and $\lim _{x \rightarrow \infty} \Omega_{\rho}(x)=\infty$.

(ii) $\Omega_{\rho}(x)$ is always increasing, and its slope satisfies $\rho<\Omega_{\rho}^{\prime}(x)<\rho+M^{2}$ for all $x \in \mathbb{R}$.

\section{Appendix B Proofs of Theorems 2-6}

Proof of Theorem 2. We provide a constructive proof that there exist discontinuous price functions described in Section 3. We derive the relationship between level sets and the price realizations for a large class of price functions in four steps: First, we formally derive the belief of uninformed agents if they learn something in addition to the usual GS information, i.e., if the price reveals both the linear combination of signal $s$ and the noisy supply $u$, and that $s$ is in a certain segment of the real line. Second, given the belief, we derive the demand function of uninformed traders. Third, we apply market clearing to obtain an implicit equation that any equilibrium price function must satisfy. Fourth, we show existence of an equilibrium by choosing level sets to be half-lines in a particular form so the attained prices produce a valid price function, and provide equilibrium prices in closed form in this equilibrium.

Posterior distribution. In the standard GS model (see also Section 2), uninformed agents have prior $d \sim N\left(0, \sigma_{d}^{2}\right)$, and infer another piece of information from the price in the form of the linear combination $l=s-C u$, where $s=d+\varepsilon, \varepsilon \sim N\left(0, \sigma_{\varepsilon}^{2}\right), u \sim N\left(0, \sigma_{u}^{2}\right)$, and $C \in \mathbb{R}^{+}$is given in Theorem 1. Standard Bayesian updating then implies that the posterior distribution is $\left.d\right|_{s-C u=l} \sim N\left(\beta_{l} l, \sigma_{d \mid l}^{2}\right)$ with

$$
\beta_{l}=\frac{\sigma_{d}^{2}}{\sigma_{d}^{2}+\sigma_{\varepsilon}^{2}+C^{2} \sigma_{u}^{2}} \text { and } \sigma_{d \mid l}^{2}=\beta_{l}\left(\sigma_{\varepsilon}^{2}+C^{2} \sigma_{u}^{2}\right)
$$

as in (10). ${ }^{21}$ Put differently, the posterior pdf is

$$
f_{d \mid l}(d \mid s-C u=l)=\frac{1}{\sigma_{d \mid l}} \phi\left(\frac{d-\beta_{l} l}{\sigma_{d \mid l}}\right) .
$$

Suppose now that besides the linear combination $l$, uninformed agents also learn that $s \in \mathcal{S} \equiv\left(\bar{s}_{1}, \bar{s}_{2}\right)$ for arbitrary $\bar{s}_{1}, \bar{s}_{2} \in \mathbb{R} \cup\{-\infty, \infty\}$ and $\bar{s}_{1}<\bar{s}_{2}$. Since the priors are jointly

\footnotetext{
${ }^{21}$ To simplify our formulas, in the appendix we denote the conditional variance $\operatorname{Var}[. \mid \mathcal{I}]$ by $\sigma_{. \mid \mathcal{I}}^{2}$.
} 
normal, the posterior distribution conditional on these two additional pieces of information becomes truncated normal. After some algebra, we obtain that the posterior pdf is given by

$$
f_{d \mid \mathcal{L}}(d \mid \mathcal{L})=\frac{1}{\sigma_{d \mid l}} \phi\left(\frac{d-\beta_{l} l}{\sigma_{d \mid l}}\right) \frac{\Phi\left(\frac{\sigma_{l \mid d}}{\sigma_{l \mid s} \sigma_{\varepsilon}}\left(\bar{s}_{2}-\frac{\sigma_{l \mid s}^{2} d+\sigma_{\varepsilon}^{2} l}{\sigma_{l \mid d}^{2}}\right)\right)-\Phi\left(\frac{\sigma_{l \mid d}}{\sigma_{l \mid s} \sigma_{\varepsilon}}\left(\bar{s}_{1}-\frac{\sigma_{l \mid s}^{2} d+\sigma_{\varepsilon}^{2} l}{\sigma_{l \mid d}^{2}}\right)\right)}{\Phi\left(\frac{1}{\theta}\left(\bar{s}_{2}-\frac{\sigma_{s}^{2}}{\sigma_{l}^{2} l} l\right)\right)-\Phi\left(\frac{1}{\theta}\left(\bar{s}_{1}-\frac{\sigma_{s}^{2}}{\sigma_{l}^{2}} l\right)\right)},
$$

where we introduce the notation $\mathcal{L}$ for the information set $\{s-C u=l, s \in \mathcal{S}\}$, and define

$$
\sigma_{l \mid s}^{2}=C^{2} \sigma_{u}^{2}, \sigma_{l \mid d}^{2}=\sigma_{\varepsilon}^{2}+C^{2} \sigma_{u}^{2}, \sigma_{s}^{2}=\sigma_{d}^{2}+\sigma_{\varepsilon}^{2}, \sigma_{l}^{2}=\sigma_{d}^{2}+\sigma_{\varepsilon}^{2}+C^{2} \sigma_{u}^{2}, \text { and } \theta=\frac{\sigma_{l \mid s} \sigma_{s}}{\sigma_{l}}=\sigma_{s \mid l} .
$$

Notice that if we fix $l$ and pick $\bar{s}_{1}=-\infty$ and $\bar{s}_{2}=\infty$, uninformed agents' belief becomes $\left.d\right|_{s-C u=l} \sim N\left(\beta_{l} l, \sigma_{d \mid l}^{2}\right)$, i.e., the posterior belief in the standard linear equilibrium.

Naturally, the above formula (and thus the results below) would still hold if we had a closed or half-closed-half-open interval instead of an open one. Moreover, (A-3) can easily be extended to the case when $\mathcal{S}$ is a disjoint union of a countable number of intervals instead of only one.

Uninformed optimization problem. Next, we solve the optimization problem

$$
\max _{x} E[-\exp \{-\alpha(d-p) x\} \mid \mathcal{L}]
$$

From (A-3) and using Lemma 8, we can show that

$$
\begin{aligned}
E[\exp (-\alpha d x) \mid \mathcal{L}] & =\exp \left\{-\alpha\left(\beta_{l} l x-\frac{1}{2} \alpha \sigma_{d \mid l}^{2} x^{2}\right)\right\} \times \\
& \times \frac{\Phi\left(\frac{1}{\theta}\left[\bar{s}_{2}-\left(\frac{\sigma_{s}^{2}}{\sigma_{l}^{2}} l-\alpha \frac{\sigma_{l \mid s}^{2} \sigma_{d}^{2}}{\sigma_{l}^{2}} x\right)\right]\right)-\Phi\left(\frac{1}{\theta}\left[\bar{s}_{1}-\left(\frac{\sigma_{s}^{2}}{\sigma_{l}^{2}} l-\alpha \frac{\sigma_{l \mid s}^{2} \sigma_{d}^{2}}{\sigma_{l}^{2}} x\right)\right]\right)}{\Phi\left(\frac{1}{\theta}\left(\bar{s}_{2}-\frac{\sigma_{s}^{2}}{\sigma_{l}^{2}} l\right)\right)-\Phi\left(\frac{1}{\theta}\left(\bar{s}_{1}-\frac{\sigma_{s}^{2}}{\sigma_{l}^{2}} l\right)\right)},
\end{aligned}
$$

hence the optimization problem (A-4) is equivalent to

$$
\begin{aligned}
\max _{x} & -\exp \left\{-\alpha\left[\left(\beta_{l} l-p\right) x-\frac{1}{2} \alpha \sigma_{d \mid l}^{2} x^{2}\right]\right\} \times \\
& \times\left[\Phi\left(\frac{1}{\theta}\left[\bar{s}_{2}-\left(\frac{\sigma_{s}^{2}}{\sigma_{l}^{2}} l-\alpha \frac{\sigma_{l \mid s}^{2} \sigma_{d}^{2}}{\sigma_{l}^{2}} x\right)\right]\right)-\Phi\left(\frac{1}{\theta}\left[\bar{s}_{1}-\left(\frac{\sigma_{s}^{2}}{\sigma_{l}^{2}} l-\alpha \frac{\sigma_{l \mid s}^{2} \sigma_{d}^{2}}{\sigma_{l}^{2}} x\right)\right]\right)\right],
\end{aligned}
$$

where the first component is the usual term that illustrates the equivalence of the CARAnormal optimization problem to a mean-variance problem, and the second term adjusts the maximand with the truncated belief. After deriving and rearranging the FOC, we obtain the following equation that optimal uninformed demand must solve for given belief and price:

$$
0=\beta_{l} l-p-\alpha \sigma_{d \mid l}^{2} x-\theta \beta_{s} \frac{\phi\left(\frac{1}{\theta}\left[\bar{s}_{2}-\left(\frac{\sigma_{s}^{2}}{\sigma_{l}^{2}} l-\alpha \frac{\sigma_{l \mid s}^{2} \sigma_{d}^{2}}{\sigma_{l}^{2}} x\right)\right]\right)-\phi\left(\frac{1}{\theta}\left[\bar{s}_{1}-\left(\frac{\sigma_{s}^{2}}{\sigma_{l}^{2}} l-\alpha \frac{\sigma_{l \mid s}^{2} \sigma_{d}^{2}}{\sigma_{l}^{2}} x\right)\right]\right)}{\Phi\left(\frac{1}{\theta}\left[\bar{s}_{2}-\left(\frac{\sigma_{s}^{2}}{\sigma_{l}^{2}} l-\alpha \frac{\sigma_{l \mid s}^{2} \sigma_{d}^{2}}{\sigma_{l}^{2}} x\right)\right]\right)-\Phi\left(\frac{1}{\theta}\left[\bar{s}_{1}-\left(\frac{\sigma_{s}^{2}}{\sigma_{l}^{2}} l-\alpha \frac{\sigma_{l \mid s}^{2} \sigma_{d}^{2}}{\sigma_{l}^{2}} x\right)\right]\right)} .
$$


The first three terms of the RHS are the same as in the linear equilibrium that lead to the optimal uninformed demand (9); the last term is the adjustment for the truncated beliefs. ${ }^{22}$

Market-clearing price. We rearrange the market-clearing condition (6) and obtain that in equilibrium we must have

$$
x_{U}=\frac{p-\beta_{s}(s-C u)}{(1-\omega) \beta_{s} C}=\frac{p-\beta_{s} l}{(1-\omega) \beta_{s} C} .
$$

With the help of (A-6), we replace the uninformed demand in (A-5). After rearranging, we obtain the following equation that the equilibrium price $p$ corresponding to the information set $\mathcal{L}$ solves:

$$
0=B l-p-\frac{\theta}{\kappa} \frac{\phi\left(\frac{1}{\theta}\left[\bar{s}_{2}+\vartheta p-\xi l\right]\right)-\phi\left(\frac{1}{\theta}\left[\bar{s}_{1}+\vartheta p-\xi l\right]\right)}{\Phi\left(\frac{1}{\theta}\left[\bar{s}_{2}+\vartheta p-\xi l\right]\right)-\Phi\left(\frac{1}{\theta}\left[\bar{s}_{1}+\vartheta p-\xi l\right]\right)}
$$

where $B$ is the same as in Theorem 1 , and

$$
\vartheta=\frac{\alpha \theta^{2}}{(1-\omega) C}, \kappa=\vartheta+\frac{1}{\beta_{s}(1-\omega)} \text { and } \xi=\kappa B-\frac{\omega}{1-\omega}
$$

with $0<\vartheta<\kappa$. Notice that setting $\left(\bar{s}_{1}, \bar{s}_{2}\right)=(-\infty, \infty)$ means the last term of the RHS of (A-7) is zero, and we get back the GS equilibrium price $p=B l$.

Closed-form expression for price with half-lines. While in general the RHS of (A-7) is a noninvertible function of $p$, it is in the two special cases we consider when $\mathcal{S}$ is either a lower or an upper half-line.

First, we assume $\mathcal{S}=\left(\bar{s}_{1}, \bar{s}_{2}\right)=(-\infty, \bar{s})$, i.e., formally, the information set of uninformed traders is $\mathcal{L}_{-}(l)=\{s-C u=l, s<\bar{s}\}$. Denoting the equilibrium price by $p_{-}$, (A-7) simplifies to

$$
0=B l-p_{-}-\frac{\theta}{\kappa} \frac{\phi\left(\frac{1}{\theta}\left[\bar{s}+\vartheta p_{-}-\xi l\right]\right)}{\Phi\left(\frac{1}{\theta}\left[\bar{s}+\vartheta p_{-}-\xi l\right]\right)} .
$$

Rearranging, we obtain

$$
\frac{\kappa}{\vartheta \theta}[(\vartheta B-\xi) l+\bar{s}]=\frac{\kappa}{\vartheta \theta}\left[\bar{s}+\vartheta p_{-}-\xi l\right]+\frac{\phi\left(\frac{1}{\theta}\left[\bar{s}+\vartheta p_{-}-\xi l\right]\right)}{\Phi\left(\frac{1}{\theta}\left[\bar{s}+\vartheta p_{-}-\xi l\right]\right)}=\Psi_{\rho}\left(\frac{1}{\theta}\left[\bar{s}+\vartheta p_{-}-\xi l\right]\right) .
$$

where $\rho \equiv \frac{\kappa-\vartheta}{\vartheta}>0$, and $\Psi_{\rho}($.$) is the strictly increasing and hence invertible function defined$ in Lemma 5. Applying $\Psi_{\rho}^{-1}$ to both sides and rearranging then yields

$$
p_{-}=\frac{\xi l-\bar{s}}{\vartheta}+\frac{\theta}{\vartheta} \Psi_{\rho}^{-1}\left(\frac{\kappa}{\vartheta \theta}[(B \vartheta-\xi) l+\bar{s}]\right) .
$$

Second, we assume $\mathcal{S}=[\bar{s}, \infty)$, i.e., the information set of uninformed agents is $\mathcal{L}_{+}(l)=$ $\{s-C u=l, s \geq \bar{s}\}$. Denoting the equilibrium price by $p_{+},(\mathrm{A}-7)$ simplifies to

$$
0=B l-p_{+}-\frac{\theta}{\kappa} \frac{-\phi\left(\frac{1}{\theta}\left[\bar{s}+\vartheta p_{+}-\xi l\right]\right)}{1-\Phi\left(\frac{1}{\theta}\left[\bar{s}+\vartheta p_{+}-\xi l\right]\right)}=B l-p_{+}+\frac{\theta}{\kappa} \frac{\phi\left(-\frac{1}{\theta}\left[\bar{s}+\vartheta p_{+}-\xi l\right]\right)}{\Phi\left(-\frac{1}{\theta}\left[\bar{s}+\vartheta p_{+}-\xi l\right]\right)}
$$

\footnotetext{
${ }^{22}$ Notice that the FOC of (A-4) is sufficient for optimality because the problem is concave: the second derivative of the expectation w.r.t. $x$ is given by $E\left[-\alpha^{2}(d-p)^{2} \exp \{-\alpha(d-p) x\} \mid \mathcal{L}\right]<0$.
} 
Performing the same steps as in the $p_{-}$case yields

$$
p_{+}=\frac{\xi l-\bar{s}}{\vartheta}-\frac{\theta}{\vartheta} \Psi_{\rho}^{-1}\left(-\frac{\kappa}{\vartheta \theta}[(B \vartheta-\xi) l+\bar{s}]\right) .
$$

Equilibrium with a linear cut. Finally, we want to choose $\bar{s}$ such that the prices obtained in (A-10) and (A-12) correspond to a valid price function, i.e., it is a bijection between the price values and the level sets of $P$.

We conjecture a linear cut of the plane into two halves by $\bar{s}(l)=\Gamma l+\Theta$, where $\Gamma, \Theta \in \mathbb{R}$ are constants to be determined. Equations (A-10) and (A-12) then become

$$
P_{-}(l) \equiv p_{-}=\frac{(\xi-\Gamma) l-\Theta}{\vartheta}+\frac{\theta}{\vartheta} \Psi_{\rho}^{-1}\left(\frac{\kappa}{\vartheta \theta}[(B \vartheta-\xi+\Gamma) l+\Theta]\right)
$$

and

$$
P_{+}(l) \equiv p_{+}=\frac{(\xi-\Gamma) l-\Theta}{\vartheta}-\frac{\theta}{\vartheta} \Psi_{\rho}^{-1}\left(-\frac{\kappa}{\vartheta \theta}[(B \vartheta-\xi+\Gamma) l+\Theta]\right),
$$

where we introduce $P_{-}(l)$ and $P_{+}(l)$ to be able to discuss the properties of the equilibrium prices $p_{-}$and $p_{+}$for different $l$ realizations, i.e. to think about them as functions of $l$. This way

$$
P(s, u) \equiv \begin{cases}P_{-}(s-C u)=P_{-}(l) & \text { if } s<\bar{s}(l) \\ P_{+}(s-C u)=P_{+}(l) & \text { if } s \geq \bar{s}(l)\end{cases}
$$

is a valid equilibrium price function if $P_{-}(l)$ and $P_{+}(l)$ are two invertible functions of $l$ and their images are disjoint. One way to satisfy these requirements is to make both $P_{-}$and $P_{+}$monotone increasing functions such that $\lim _{l \rightarrow \infty} P_{-}(l)$ and $\lim _{l \rightarrow-\infty} P_{+}(l)$ are both finite while $\lim _{l \rightarrow \infty} P_{-}(l)<\lim _{l \rightarrow-\infty} P_{+}(l)$. From (A-13) and (A-14) it is also imminent that $P_{-}$ and $P_{+}$are differentiable everywhere.

Instead of rewriting (A-13) and (A-14) to analyze $P_{-}$and $P_{+}$, we proceed from (A-9) and (A-11). We start with $P_{-}$and derive the appropriate constants $\Gamma, \Theta \in \mathbb{R}$ from there; then we look at $P_{+}$and confirm it behaves accordingly.

Substituting $\bar{s}(l)=\Gamma l+\Theta$ into (A-9) and rearranging, we obtain

$$
\frac{1}{\theta}\left[(\kappa B+\Gamma-\xi) l+\Theta-(\kappa-\vartheta) P_{-}(l)\right]=\Psi_{0}\left(\frac{1}{\theta}\left[(\Gamma-\xi) l+\Theta+\vartheta P_{-}(l)\right]\right),
$$

where in the last step we used the definition of $\Psi_{0}$. Since $P_{-}$is a function of $l,(\mathrm{~A}-15)$ has to hold for all $l$ as an identity. Hence, the derivative of the two sides w.r.t. $l$ must be that same too, that is,

$$
\frac{1}{\theta}\left[(\kappa B+\Gamma-\xi)-(\kappa-\vartheta) P_{-}^{\prime}(l)\right]=\frac{1}{\theta}\left[(\Gamma-\xi)+\vartheta P_{-}^{\prime}(l)\right] \Psi_{0}^{\prime}\left(\frac{1}{\theta}\left[(\Gamma-\xi) l+\Theta+\vartheta P_{-}(l)\right]\right) .
$$

We want to choose $\Gamma$ and $\Theta$ such that $\lim _{l \rightarrow \infty} P_{-}(l)$ is finite, which also means $\lim _{l \rightarrow \infty} P_{-}^{\prime}(l)=$ 0 . Taking the limits of both sides of (A-16), we have

$$
\frac{\kappa B+\Gamma-\xi}{\theta}=\frac{\Gamma-\xi}{\theta} \lim _{l \rightarrow \infty} \Psi_{0}^{\prime}\left(\frac{1}{\theta}\left[(\Gamma-\xi) l+\Theta+\vartheta P_{-}(l)\right]\right) .
$$


First, notice that from Lemma $4 \Psi_{0}^{\prime}$ is bounded, and thus $\Gamma=\xi$ would make the RHS zero while the LHS is not. Therefore, $\Gamma-\xi \neq 0$, and rearranging (A-17) we obtain

$$
1+\frac{\kappa B}{\Gamma-\xi}=\lim _{l \rightarrow \infty} \Psi_{0}^{\prime}\left(\frac{1}{\theta}\left[(\Gamma-\xi) l+\Theta+\vartheta P_{-}(l)\right]\right) .
$$

Moreover, $\lim _{l \rightarrow \infty} P_{-}(l)$ being finite and $\Gamma-\xi \neq 0$ together imply that

$$
\lim _{l \rightarrow \infty} \frac{1}{\theta}\left[(\Gamma-\xi) l+\Theta+\vartheta P_{-}(l)\right]= \begin{cases}-\infty & \text { if } \Gamma<\xi \\ \infty & \text { if } \Gamma>\xi\end{cases}
$$

hence from Lemma 4 we get

$$
\lim _{l \rightarrow \infty} \Psi_{0}^{\prime}\left(\frac{1}{\theta}\left[(\Gamma-\xi) l+\Theta+\vartheta P_{-}(l)\right]\right)= \begin{cases}0 & \text { if } \Gamma<\xi \\ 1 & \text { if } \Gamma>\xi\end{cases}
$$

However, $\frac{\kappa B}{\Gamma-\xi} \neq 0$ means the LHS of (A-18) cannot be 1, and thus (A-18) can only hold if $\Gamma=\xi-\kappa B<\xi$. Substituting it into (A-15), we obtain

$$
\frac{\Theta+(\vartheta-\kappa) P_{-}(l)}{\theta}=\Psi_{0}\left(\frac{-\kappa B l+\Theta+\vartheta P_{-}(l)}{\theta}\right)
$$

Taking the limit of both sides when $l \rightarrow \infty$ and using the above observations, we obtain

$$
\frac{\Theta}{\theta}+\frac{\vartheta-\kappa}{\theta} \lim _{l \rightarrow \infty} P_{-}(l)=\lim _{l \rightarrow \infty} \Psi_{0}\left(\frac{-\kappa B l+\Theta+\vartheta P_{-}(l)}{\theta}\right)=0 .
$$

Introducing the notation $D \equiv \frac{\Theta}{\kappa-\vartheta}=(1-\omega) \beta_{s} \Theta$, we obtain $\lim _{l \rightarrow \infty} P_{-}(l)=D$; the limit is indeed finite. Moreover, substituting $\Gamma=\xi-\kappa B$ and the definition of $D$ into (A-13), we obtain $P_{-}(l)=\frac{\kappa B l-(\kappa-\vartheta) D}{\vartheta}+\frac{\theta}{\vartheta} \Psi_{\rho}^{-1}\left(-\frac{\kappa \rho}{\theta}(B l-D)\right)=(1+\rho) B l-\rho D+\frac{\theta}{\vartheta} \Psi_{\rho}^{-1}\left(-\frac{\kappa \rho}{\theta}(B l-D)\right)$, which is identical to (12) if we note that

$$
\rho=\frac{\kappa-\vartheta}{\vartheta}, \zeta=\frac{\theta}{\vartheta}, \text { and } \lambda=\frac{\kappa \rho}{\theta}
$$

all positive constants. Moreover, after some algebra we also get that $\Gamma=\xi-\kappa B$ simplifies to $\Gamma=-\frac{\omega}{1-\omega}$, as in (11).

Finally, to show that $P_{-}$is a monotone function, we differentiate (A-20) w.r.t $l$. Using $\Gamma=\xi-\kappa B$ and rearranging,

$$
P_{-}^{\prime}(l)=(1+\rho) B\left(1-\frac{\rho}{\Psi_{\rho}^{\prime}\left(\Psi_{\rho}^{-1}\left(-\frac{\kappa \rho}{\theta}(B l-D)\right)\right)}\right) .
$$

But Lemma 5 implies $\rho<\Psi_{\rho}^{\prime}(x)$ for all $x \in \mathbb{R}$, so $1-\frac{\rho}{\Psi_{\rho}^{\prime}(x)}>0$ for all $x \in \mathbb{R}$, and hence $P_{-}(l)$ is strictly increasing for all $l \in \mathbb{R}$, thus invertible. 
For $P_{+}$, we first substitute $\Gamma=\xi-\kappa B$ into (A-14) and differentiate to obtain

$$
P_{+}^{\prime}(l)=(1+\rho) B\left(1-\frac{\rho}{\Psi_{\rho}^{\prime}\left(\Psi_{\rho}^{-1}\left(\frac{1}{\theta}\left(\rho \kappa B l-\frac{\kappa \Theta}{\vartheta}\right)\right)\right)}\right) .
$$

Lemma 5 implies $\rho<\Psi_{\rho}^{\prime}(x)$ for all $x \in \mathbb{R}$, so $1-\frac{\rho}{\Psi_{\rho}^{\prime}(x)}>0$ for all $x \in \mathbb{R}$, and hence $P_{+}(l)$ is monotone increasing for all $l \in \mathbb{R}$, thus invertible. Moreover, due to monotonicity, the

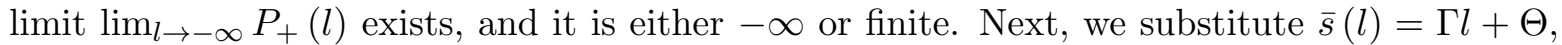
$\Gamma=\xi-\kappa B$, and $D=\frac{\Theta}{\kappa-\vartheta}$ into (A-11) and rearrange to obtain a form similar to (A-19):

$$
\frac{(\kappa-\vartheta) P_{+}(l)-\Theta}{\theta}=\Psi_{0}\left(\frac{\kappa B l-\Theta-\vartheta P_{+}(l)}{\theta}\right) .
$$

Taking the limit of both sides when $l \rightarrow-\infty$ and using the above observations, we obtain

$$
\frac{\kappa-\vartheta}{\theta} \lim _{l \rightarrow-\infty} P_{+}(l)-\frac{\Theta}{\theta}=\lim _{l \rightarrow-\infty} \Psi_{0}\left(\frac{\kappa B l-\Theta-\vartheta P_{+}(l)}{\theta}\right) .
$$

Notice that $\lim _{l \rightarrow-\infty} P_{+}(l)=-\infty$ would imply that the LHS of this equation is $-\infty$ due to $\frac{\kappa-\vartheta}{\theta}>0$, which cannot happen because $\Psi_{0}(x)>0$ for all $x \in \mathbb{R}$ and hence the RHS of the equation must be non-negative. Hence, we must have a finite $\lim _{l \rightarrow-\infty} P_{+}(l)$. In this case, however, the RHS simplifies to

$$
\lim _{l \rightarrow-\infty} \Psi_{0}\left(\frac{\kappa B l-\Theta-\vartheta P_{+}(l)}{\theta}\right)=\lim _{x \rightarrow-\infty} \Psi_{0}(x)=0,
$$

and plugging it back to (A-25) we obtain

$$
\lim _{l \rightarrow-\infty} P_{+}(l)=\frac{\Theta}{\kappa-\vartheta}=D=\lim _{l \rightarrow \infty} P_{-}(l) .
$$

Therefore, both $P_{-}$and $P_{+}$are increasing functions and there is no overlap in their images. Hence, $P_{L C}$ is a valid price function. Substituting $\Gamma=\xi-\kappa B$ and $D=\frac{\Theta}{\kappa-\vartheta}$ into (A-14) then yields (13), and concludes the proof of Theorem 2.

Proof of Theorem 3. Let us suppose that in equilibrium uninformed agents learn both the linear combination $l=s-C u$ and that $s$ and $u$ satisfy $D_{n} / \beta_{s} \leq s-\omega C u<D_{n+1} / \beta_{s}$ with $D_{n}<D_{n+1}$, or, alternatively, that $s \in \mathcal{S}_{n}=\left[\bar{s}_{n}(l), \bar{s}_{n+1}(l)\right)$ with $\bar{s}_{n}(l)=\frac{D_{n}-\omega \beta_{s} l}{(1-\omega) \beta_{s}}$. The posterior pdf of uninformed agents' belief is then given, just as in (A-3), by

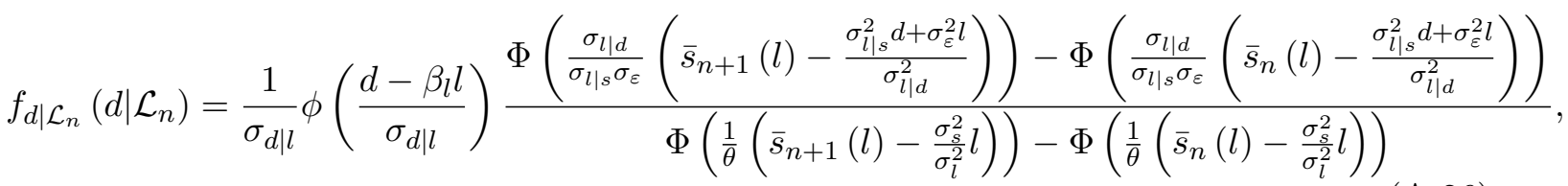

where $\mathcal{L}_{n}$ denotes the information set $\left\{s-C u=l, s \in \mathcal{S}_{n}\right\}$. After the same steps as in the proof of Theorem 2, i.e., solving the uninformed optimization problem and clearing the market, we obtain a result identical to (A-7): $P_{n}(l)$ must satisfy the following equation for all $l$ :

$$
0=B l-P_{n}(l)-\frac{\theta}{\kappa} \frac{\phi\left(\frac{1}{\theta}\left[\bar{s}_{n+1}(l)+\vartheta P_{n}(l)-\xi l\right]\right)-\phi\left(\frac{1}{\theta}\left[\bar{s}_{n}(l)+\vartheta P_{n}(l)-\xi l\right]\right)}{\Phi\left(\frac{1}{\theta}\left[\bar{s}_{n+1}(l)+\vartheta P_{n}(l)-\xi l\right]\right)-\Phi\left(\frac{1}{\theta}\left[\bar{s}_{n}(l)+\vartheta P_{n}(l)-\xi l\right]\right)},
$$


where $\vartheta, \kappa$ and $\xi$ are given in (A-8). After some algebra, we rewrite (A-27) as

$$
\begin{aligned}
& -\frac{1}{\theta} \kappa \rho B l=(1+\rho) \frac{1}{\theta}\left[-\frac{\omega}{1-\omega} l+\vartheta P_{n}(l)-\xi l\right] \\
& \quad+\frac{\phi\left(\frac{1}{\theta}\left[\bar{s}_{n+1}(l)+\vartheta P_{n}(l)-\xi l\right]\right)-\phi\left(\frac{1}{\theta}\left[\bar{s}_{n}(l)+\vartheta P_{n}(l)-\xi l\right]\right)}{\Phi\left(\frac{1}{\theta}\left[\bar{s}_{n+1}(l)+\vartheta P_{n}(l)-\xi l\right]\right)-\Phi\left(\frac{1}{\theta}\left[\bar{s}_{n}(l)+\vartheta P_{n}(l)-\xi l\right]\right)} .
\end{aligned}
$$

From the definition of $\bar{s}_{n}(l)$ notice that the term $\frac{1}{\theta}\left[-\frac{\omega}{1-\omega} l+\vartheta P_{n}(l)-\xi l\right]$ and the arguments of $\phi($.$) and \Phi($.$) on the RHS of (A-28) only differ in additive constants, prompting the notation$

$$
\mathrm{K}(l)=\frac{1}{\theta}\left[-\frac{\omega}{1-\omega} l+\vartheta P_{n}(l)-\xi l\right]+\frac{1}{2 \theta} \frac{D_{n}+D_{n+1}}{(1-\omega) \beta_{s}} \text { and } M=\frac{1}{2 \theta} \frac{D_{n+1}-D_{n}}{(1-\omega) \beta_{s}},
$$

which, due to (A-8), simplify to

$$
\mathrm{K}(l)=\frac{1}{\theta}\left[-\kappa B l+\vartheta P_{n}(l)\right]+\frac{\kappa-\vartheta}{\theta} \frac{D_{n}+D_{n+1}}{2} \text { and } M=\frac{\kappa-\vartheta}{\theta} \frac{D_{n+1}-D_{n}}{2}>0 .
$$

Applying (A-8), the definition of $\rho$, and (A-29), after some algebra (A-28) becomes

$$
-\frac{\kappa \rho}{\theta}\left(B l-\frac{D_{n+1}+D_{n}}{2}\right)=(1+\rho) \mathrm{K}(l)+\frac{\phi(\mathrm{K}(l)+M)-\phi(\mathrm{K}(l)-M)}{\Phi(\mathrm{K}(l)+M)-\Phi(\mathrm{K}(l)-M)}=\Omega_{\rho}(\mathrm{K}(l)),
$$

where the last equality follows from the definition of $\Omega_{\rho}(x)$ in (A-2). Part (ii) of Lemma 6 states that $\Omega_{\rho}$ is an increasing function that takes all real values if $\rho>0$, hence, we can apply its inverse on both sides of (A-30) to obtain

$$
\Omega_{\rho}^{-1}\left(-\frac{\kappa \rho}{\theta}\left(B l-\frac{D_{n+1}+D_{n}}{2}\right)\right)=\mathrm{K}(l) .
$$

Combining the last equation with (A-29), after some algebra and using the notation (A-21), we get

$$
P_{n}(l)=(1+\rho) B l-\rho \frac{D_{n}+D_{n+1}}{2}+\zeta \Omega_{\rho}^{-1}\left(-\lambda\left(B l-\frac{D_{n+1}+D_{n}}{2}\right)\right)
$$

as a closed-form expression for $P_{n}(l)$.

For the monotonicity of $P_{n}$, we differentiate (A-31) and simplify, which yields

$$
P_{n}^{\prime}(l)=(1+\rho) B\left(1-\frac{\rho}{\Omega_{\rho}^{\prime}\left(\Omega_{\rho}^{-1}\left(-\frac{\kappa \rho}{\theta}\left(B l-\frac{D_{n+1}+D_{n}}{2}\right)\right)\right)}\right) .
$$

But part (ii) of Lemma 6 states that $\Omega_{\rho}^{\prime}(x)>\rho$ for all $x \in \mathbb{R}$, which implies $P_{n}^{\prime}(l)>0$ for all $l \in \mathbb{R}$, i.e., $P_{n}$ is an increasing function.

Next we rearrange (A-30) as

$$
-\frac{\kappa-\vartheta}{\theta}\left(P_{n}(l)-\frac{D_{n}+D_{n+1}}{2}\right)=\mathrm{K}(l)+\frac{\phi(K(l)+M)-\phi(K(l)-M)}{\Phi(K(l)+M)-\Phi(K(l)-M)}=\Omega_{0}(\mathrm{~K}(l)) .
$$


Part (ii) of Lemma 6 states that $-M<\Omega_{0}(x)<M$ for all $x \in \mathbb{R}$, hence,from (A-29) and (A-32), we can write

$$
-M<-\frac{\kappa-\vartheta}{\theta}\left(P_{n}(l)-\frac{D_{n}+D_{n+1}}{2}\right)<M .
$$

After some algebra, this is equivalent to $D_{n}<P_{n}(l)<D_{n+1}$. As long as $D_{n+1}$ is finite, $P_{n}$ is bounded from above, but it is also increasing, which implies $\lim _{l \rightarrow \infty} P_{n}(l)$ exists and is finite (at most $\left.D_{n+1}\right)$. But then from (A-29) it must be $\lim _{l \rightarrow \infty} \mathrm{K}(l)=-\infty$. Rearranging (A-32) as

$$
P_{n}(l)=\frac{D_{n}+D_{n+1}}{2}-\frac{\theta}{\kappa-\vartheta} \Omega_{0}(\mathrm{~K}(l)),
$$

taking the limit $l \rightarrow \infty$ of both sides, and using part (i) of Lemma 6, we end up with

$$
\lim _{l \rightarrow \infty} P_{n}(l)=\frac{D_{n}+D_{n+1}}{2}-\frac{\theta}{\kappa-\vartheta} \lim _{l \rightarrow \infty} \Omega_{0}(\mathrm{~K}(l))=\frac{D_{n}+D_{n+1}}{2}-\frac{\theta}{\kappa-\vartheta}(-M)=D_{n+1} .
$$

Similarly, we can show that $\lim _{l \rightarrow-\infty} P_{n}(l)=D_{n}$, which concludes the proof of Theorem 3 .

Proof of Theorem 4. The first part of the theorem is straightforward: from the equilibrium form $\bar{s}_{n}(l)=\frac{D_{n}-\omega \beta_{s} l}{(1-\omega) \beta_{s}}$ it is imminent that $\bar{s}_{n+1}(l)-\bar{s}_{n}(l)=\frac{D_{n+1}-D_{n}}{(1-\omega) \beta_{s}}$. Therefore, for any $\epsilon>0$ choosing a partition in which $D_{n+1}-D_{n}<(1-\omega) \beta_{s} \epsilon$ for all $n \in \mathbb{Z}$ will imply that $\Delta s=\sup _{n}\left[\bar{s}_{n+1}(l)-\bar{s}_{n}(l)\right]<\epsilon$.

For the second part of the theorem, to express the conditional variance $\operatorname{Var}[d \mid p]$, which is equivalent to $\operatorname{Var}\left[d \mid \mathcal{L}_{n}\right]=\operatorname{Var}\left[d \mid l, s \in \mathcal{S}_{n}\right]$ when $D_{n}<p=P_{n}(l)<D_{n+1}$, we need to do calculations similar to those in the proof of Proposition 2. As

$$
\operatorname{Var}\left[d \mid \mathcal{L}_{n}\right]=E\left[d^{2} \mid \mathcal{L}_{n}\right]-E^{2}\left[d \mid \mathcal{L}_{n}\right],
$$

we first need to determine the expectations. From (A-26), we can write

$$
\begin{gathered}
E\left[d \mid \mathcal{L}_{n}\right]=\int_{-\infty}^{\infty} d f_{d \mid \mathcal{L}_{n}}\left(d \mid \mathcal{L}_{n}\right) \mathrm{d} d=\frac{\int_{-\infty}^{\infty} d \frac{1}{\sigma_{d \mid l}} \phi\left(\frac{1}{\sigma_{d \mid l}}\left(d-\beta_{l} l\right)\right) \Phi\left(-\frac{\sigma_{l \mid s}}{\sigma_{l \mid d} \sigma_{\varepsilon}}\left(d-\frac{\sigma_{l \mid d}^{2} \bar{s}_{n+1}-\sigma_{\varepsilon}^{2} l}{\sigma_{l \mid s}^{2}}\right)\right) \mathrm{d} d}{\Phi\left(\frac{1}{\theta}\left(\bar{s}_{n+1}-\frac{\sigma_{s}^{2}}{\sigma_{l}^{2}} l\right)\right)-\Phi\left(\frac{1}{\theta}\left(\bar{s}_{n}-\frac{\sigma_{s}^{2}}{\sigma_{l}^{2} l}\right)\right)} \\
-\frac{\int_{-\infty}^{\infty} d \frac{1}{\sigma_{d \mid l}} \phi\left(\frac{1}{\sigma_{d \mid l}}\left(d-\beta_{l} l\right)\right) \Phi\left(-\frac{\sigma_{l \mid s}}{\sigma_{l \mid d} \sigma_{\varepsilon}}\left(d-\frac{\sigma_{l \mid d}^{2} \bar{s}_{n}-\sigma_{\varepsilon}^{2} l}{\sigma_{l \mid s}^{2}}\right)\right) \mathrm{d} d}{\Phi\left(\frac{1}{\theta}\left(\bar{s}_{n+1}-\frac{\sigma_{s}^{2}}{\sigma_{l}^{2}} l\right)\right)-\Phi\left(\frac{1}{\theta}\left(\bar{s}_{n}-\frac{\left.\left.\sigma_{s}^{2} l\right)\right)}{\sigma_{l}^{2} l}\right)\right.} .
\end{gathered}
$$

To express the two integrals in closed form, we use Lemma 9 for

$$
X=\frac{1}{\sigma_{d \mid l}}, x=\beta_{l} l, Y=-\frac{\sigma_{l \mid s}}{\sigma_{l \mid d} \sigma_{\varepsilon}}, \text { and } y=\frac{\sigma_{l \mid d}^{2} \bar{s}_{n+1}-\sigma_{\varepsilon}^{2} l}{\sigma_{l \mid s}^{2}}
$$

and

$$
X=\frac{1}{\sigma_{d \mid l}}, x=\beta_{l} l, Y=-\frac{\sigma_{l \mid s}}{\sigma_{l \mid d} \sigma_{\varepsilon}}, \text { and } y=\frac{\sigma_{l \mid d}^{2} \bar{s}_{n}-\sigma_{\varepsilon}^{2} l}{\sigma_{l \mid s}^{2}}
$$


respectively. After rearranging the result, we obtain

$$
E\left[d \mid \mathcal{L}_{n}\right]=\beta_{l} l-\sqrt{\sigma_{d \mid l}^{2}-\sigma_{d \mid l}^{2}} \frac{\phi\left(\frac{1}{\theta}\left(\bar{s}_{n+1}-\frac{\sigma_{s}^{2}}{\sigma_{l}^{2}} l\right)\right)-\phi\left(\frac{1}{\theta}\left(\bar{s}_{n}-\frac{\sigma_{s}^{2}}{\sigma_{l}^{2}} l\right)\right)}{\Phi\left(\frac{1}{\theta}\left(\bar{s}_{n+1}-\frac{\sigma_{s}^{2}}{\sigma_{l}^{2}} l\right)\right)-\Phi\left(\frac{1}{\theta}\left(\bar{s}_{n}-\frac{\sigma_{s}^{2}}{\sigma_{l}^{2}} l\right)\right)} .
$$

Similarly, we express the expectation of the squared payoff:

$$
\begin{gathered}
E\left[d^{2} \mid \mathcal{L}_{n}\right]=\int_{-\infty}^{\infty} d^{2} f_{d \mid \mathcal{L}_{n}}\left(d \mid \mathcal{L}_{n}\right) \mathrm{d} d=\frac{\int_{-\infty}^{\infty} d^{2} \frac{1}{\sigma_{d \mid l}} \phi\left(\frac{1}{\sigma_{d \mid l}}\left(d-\beta_{l} l\right)\right) \Phi\left(-\frac{\sigma_{l \mid s}}{\sigma_{l \mid d} \sigma_{\varepsilon}}\left(d-\frac{\sigma_{l \mid d^{2}}^{2} \bar{s}_{n+1}-\sigma_{\varepsilon}^{2} l}{\sigma_{l \mid s}^{2}}\right)\right) \mathrm{d} d}{\Phi\left(\frac{1}{\theta}\left(\bar{s}_{n+1}-\frac{\sigma_{s}^{2} l}{\sigma_{l}^{2}} l\right)\right)-\Phi\left(\frac{1}{\theta}\left(\bar{s}_{n}-\frac{\sigma_{s}^{2} l}{\sigma_{l}^{2}} l\right)\right)} \\
-\frac{\int_{-\infty}^{\infty} d^{2} \frac{1}{\sigma_{d \mid l}} \phi\left(\frac{1}{\sigma_{d \mid l}}\left(d-\beta_{l} l\right)\right) \Phi\left(-\frac{\sigma_{l \mid s}}{\sigma_{l \mid d} \sigma_{\varepsilon}}\left(d-\frac{\sigma_{l \mid d}^{2} \bar{s}_{n}-\sigma_{\varepsilon}^{2} l}{\sigma_{l \mid s}^{2}}\right)\right) \mathrm{d} d}{\Phi\left(\frac{1}{\theta}\left(\bar{s}_{n+1}-\frac{\sigma_{s}^{2}}{\sigma_{l}^{2}} l\right)\right)-\Phi\left(\frac{1}{\theta}\left(\bar{s}_{n}-\frac{\sigma_{s}^{2}}{\sigma_{l}^{2} l}\right)\right)},
\end{gathered}
$$

and to express the two integrals in closed form, we use Lemma 10 for (A-34) and (A-35), respectively. Substituting the expression we obtain this way into (A-33), using (A-36), rearranging, and using the simplifying notation

$$
\Pi(l)=\frac{1}{2}\left[\frac{1}{\theta}\left(\bar{s}_{n+1}-\frac{\sigma_{s}^{2}}{\sigma_{l}^{2}} l\right)+\frac{1}{\theta}\left(\bar{s}_{n}-\frac{\sigma_{s}^{2}}{\sigma_{l}^{2}} l\right)\right]=-\frac{1}{\theta}\left(\frac{\omega}{1-\omega}+\frac{\sigma_{s}^{2}}{\sigma_{l}^{2}}\right) l+\frac{\kappa-\vartheta}{\theta} \frac{D_{n+1}+D_{n}}{2}
$$

and

$$
M=\frac{1}{2}\left[\frac{1}{\theta}\left(\bar{s}_{n+1}-\frac{\sigma_{s}^{2}}{\sigma_{l}^{2}} l\right)-\frac{1}{\theta}\left(\bar{s}_{n}-\frac{\sigma_{s}^{2}}{\sigma_{l}^{2}} l\right)\right]=\frac{\kappa-\vartheta}{\theta} \frac{D_{n+1}-D_{n}}{2},
$$

where $M$ is the same as in (A-29), we obtain

$$
\begin{gathered}
\operatorname{Var}\left[d \mid l, s \in \mathcal{S}_{n}\right]=\sigma_{d \mid l}^{2}-\left(\sigma_{d \mid l}^{2}-\sigma_{d \mid s}^{2}\right) \frac{(\Pi(l)+M) \phi(\Pi(l)+M)-(\Pi(l)-M) \phi(\Pi(l)-M)}{\Phi(\Pi(l)+M)-\Phi(\Pi(l)-M)} \\
-\left(\sigma_{d \mid l}^{2}-\sigma_{d \mid s}^{2}\right)\left(\frac{\phi(\Pi(l)+M)-\phi(\Pi(l)-M)}{\Phi(\Pi(l)+M)-\Phi(\Pi(l)-M)}\right)^{2}=\sigma_{d \mid s}^{2}+\left(\sigma_{d \mid l}^{2}-\sigma_{d \mid s}^{2}\right) \Omega_{0}^{\prime}(\Pi(l)),
\end{gathered}
$$

where the second equality recognizes the derivative of $\Omega_{0}(x)$; see, e.g., (OA-6). Since part (ii) of Lemma 6 states that $\Omega_{0}^{\prime}(x)<M^{2}$ for all $x \in \mathbb{R}$, (A-37) implies $\operatorname{Var}\left[d \mid l, s \in \mathcal{S}_{n}\right]<$ $\sigma_{d \mid s}^{2}+\left(\sigma_{d \mid l}^{2}-\sigma_{d \mid s}^{2}\right) M^{2}$ for all $l \in \mathbb{R}$, and thus we have obtained that

$$
\operatorname{Var}[d \mid p]-\sigma_{d \mid s}^{2}<\left(\sigma_{d \mid l}^{2}-\sigma_{d \mid s}^{2}\right) M^{2}=\frac{1}{4}\left(\sigma_{d \mid l}^{2}-\sigma_{d \mid s}^{2}\right)\left(\frac{\kappa-\vartheta}{\theta}\right)^{2}\left(D_{n+1}-D_{n}\right)^{2}
$$

for all prices $D_{n}<p<D_{n+1}$. But then for any $\delta>0$ choosing a partition of the state space that satisfies

$$
D_{n+1}-D_{n} \leq \frac{2}{\frac{\kappa-\vartheta}{\theta} \sqrt{\sigma_{d \mid l}^{2}-\sigma_{d \mid s}^{2}}} \sqrt{\delta}
$$

for all $n \in \mathbb{Z}$ will provide an equilibrium in which $\Delta \operatorname{Var}=\sup _{p} \operatorname{Var}[d \mid p]-\sigma_{d \mid s}^{2}<\delta$, i.e., the uncertainty that uninformed traders face is only $\delta$ larger than the uncertainty of informed traders. This completes the proof of Theorem 4. 
Proof of Theorems 5 and 6 . We follow the same steps as in the GS case.

Posterior distribution. In the standard differential information model (Hellwig (1980), Diamond and Verrecchia (1981), Admati (1985)), agent $i$ has prior $d \sim N\left(0, \sigma_{d}^{2}\right)$, observes the private signal $s_{i}=d+\varepsilon_{i}, \varepsilon_{i} \sim N\left(0, \sigma_{\varepsilon}^{2}\right)$, and infers another piece of information from the price in the form of the linear combination $l=d-G u$, where $u \sim N\left(0, \sigma_{u}^{2}\right)$ and $G \in \mathbb{R}^{+}$. Standard Bayesian updating then implies that the posterior distribution is $\left.d\right|_{s_{i}, d-G u=l} \sim$ $N\left(\gamma_{s} s_{i}+\gamma_{l} l, \sigma_{d \mid s, l}^{2}\right)$ with

$$
\gamma_{s}=\frac{\sigma_{d}^{2} G^{2} \sigma_{u}^{2}}{\sigma_{d}^{2} G^{2} \sigma_{u}^{2}+\sigma_{d}^{2} \sigma_{\varepsilon}^{2}+\sigma_{\varepsilon}^{2} G^{2} \sigma_{u}^{2}}, \gamma_{l}=\frac{\sigma_{d}^{2} \sigma_{\varepsilon}^{2}}{\sigma_{d}^{2} G^{2} \sigma_{u}^{2}+\sigma_{d}^{2} \sigma_{\varepsilon}^{2}+\sigma_{\varepsilon}^{2} G^{2} \sigma_{u}^{2}}, \text { and } \sigma_{d \mid s, l}^{2}=\gamma_{s} \sigma_{\varepsilon}^{2} ;
$$

that is, the posterior pdf is

$$
f_{d \mid s_{i}, d-G u}\left(d \mid s_{i}, l\right)=\frac{1}{\sigma_{d \mid s, l}} \phi\left(\frac{d-\left(\gamma_{s} s_{i}+\gamma_{l} l\right)}{\sigma_{d \mid s, l}}\right) .
$$

Suppose now that besides the linear combination $l$, agent $i$ also learns that $d \in \mathcal{D} \equiv\left(\bar{d}_{1}, \bar{d}_{2}\right)$ for $\bar{d}_{1}, \bar{d}_{2} \in \mathbb{R} \cup\{-\infty, \infty\}$ and $\bar{d}_{1}<\bar{d}_{2}$. Since the priors on $d, \varepsilon_{i}$ and $u$ are normal, the posterior distribution conditional on all the information available to agent $i$ is a generalization of the truncated normal distribution, and it is easy to show that has pdf

$$
f_{d \mid \mathcal{L}_{i}}\left(d \mid \mathcal{L}_{i}\right)=1_{d \in \mathcal{D}} \frac{1}{\sigma_{d \mid s, l}} \frac{\phi\left(\frac{d-\left(\gamma_{s} s_{i}+\gamma_{l} l\right)}{\sigma_{d \mid s, l}}\right)}{\Phi\left(\frac{\bar{d}_{2 i}-\left(\gamma_{s} s_{i}+\gamma_{l} l\right)}{\sigma_{d \mid s, l}}\right)-\Phi\left(\frac{\bar{d}_{2 i-1}-\left(\gamma_{s} s_{i}+\gamma_{l} l\right)}{\sigma_{d \mid s, l}}\right)},
$$

where we introduce the simplifying notation $\mathcal{L}_{i}$ for the information set $\left\{s_{i}, d-G u=l, d \in \mathcal{D}\right\}$. Notice that if we fix $l$ and pick $\bar{d}_{1}=-\infty$ and $\bar{d}_{2}=\infty$, agent $i$ 's belief becomes $\left.d\right|_{s_{t}, l} \sim$ $N\left(\gamma_{s} s_{i}+\gamma_{l} l, \sigma_{d \mid s, l}^{2}\right)$, i.e., the posterior belief in the standard linear equilibrium. Naturally, the formula above (and thus the results below) would still hold if we had a closed or a halfopen-half-closed interval instead of an open one. Moreover, (A-38) can easily be extended to the case when $\mathcal{D}$ is a disjoint union of a countable number of intervals instead of only one.

Optimization problems. Next, we solve the optimization problem

$$
\max _{x} E\left[-\exp (-\alpha(d-p) x) \mid \mathcal{L}_{i}\right]
$$

From (A-38), after some algebra we show that the optimization problem (A-39) is equivalent to

$$
\begin{aligned}
& \max _{x}-\exp \left\{-\alpha\left[\left(\gamma_{s} s_{i}+\gamma_{l} l-p\right) x-\frac{1}{2} \alpha \sigma_{d \mid s, l}^{2} x^{2}\right]\right\} \times \\
& \quad \times\left[\Phi\left(\frac{1}{\sigma_{d \mid s, l}}\left[\bar{d}_{2}-\left(\gamma_{s} s_{i}+\gamma_{l} l-\alpha \sigma_{d \mid s, l}^{2} x\right)\right]\right)-\Phi\left(\frac{1}{\sigma_{d \mid s, l}}\left[\bar{d}_{1}-\left(\left(\gamma_{s} s_{i}+\gamma_{l} l\right)-\alpha \sigma_{d \mid s, l}^{2} x\right)\right]\right)\right],
\end{aligned}
$$

where the first component is the usual term that illustrates the CARA-normal optimization problem is equivalent to a mean-variance problem, and the second term adjusts the maximand 
with the 'truncated' belief. After deriving and rearranging the FOC, we obtain the inverse demand function of agent $i$ :

$$
0=\gamma_{s} s_{i}+\gamma_{l} l-p-\alpha \sigma_{d \mid s, l}^{2} x_{i}-\sigma_{d \mid s, l} \frac{\phi\left(\frac{\bar{d}_{2}-\left(\gamma_{s} s_{i}+\gamma_{l} l-\alpha \sigma_{d \mid s, l}^{2} x_{i}\right)}{\sigma_{d \mid s, l}}\right)-\phi\left(\frac{\bar{d}_{1}-\left(\gamma_{s} s_{i}+\gamma_{l} l-\alpha \sigma_{d \mid s, l}^{2} x_{i}\right)}{\sigma_{d \mid s, l}}\right)}{\Phi\left(\frac{\bar{d}_{2}-\left(\gamma_{s} s_{i}+\gamma_{l} l-\alpha \sigma_{d \mid s, l}^{2} x_{i}\right)}{\sigma_{d \mid s, l}}\right)-\Phi\left(\frac{\bar{d}_{1}-\left(\gamma_{s} s_{i}+\gamma_{l} l-\alpha \sigma_{d \mid s, l}^{2} x_{i}\right)}{\sigma_{d \mid s, l}}\right)} .
$$

Optimal demand and equilibrium price in the linear equilibrium. In the standard linear equilibrium where agents only learn the linear combination $l$ from the market price we have $\bar{d}_{1}=-\infty$ and $\bar{d}_{2}=\infty$. Substituting these values into (A-40) and rearranging, we obtain that agent $i$ 's optimal demand curve is

$$
x_{i}=\frac{\gamma_{s} s_{i}+\gamma_{l} l-p}{\alpha \sigma_{d \mid s, l}^{2}} .
$$

Imposing market clearing, we get

$$
u=\int x_{i}\left(s_{i}, p\right) \mathrm{d} i=\int \frac{\gamma_{s} s_{i}+\gamma_{l} l-p}{\alpha \sigma_{d \mid s, l}^{2}} \mathrm{~d} i=\frac{\gamma_{s} d+\gamma_{l} l-p}{\alpha \sigma_{d \mid s, l}^{2}},
$$

that is

$$
p=\gamma_{s}\left(d-\frac{\alpha \sigma_{d \mid s, l}^{2}}{\gamma_{s}} u\right)+\gamma_{l} l
$$

Thus, the equilibrium price only depends on $d$ and $u$ through $l=d-G u$ iff $G=\alpha \sigma_{d \mid s, l}^{2} / \gamma_{s}=$ $\alpha \sigma_{\varepsilon}^{2}$. Substituting it back into (A-41) we obtain $p=\left(\gamma_{s}+\gamma_{l}\right) l$, which yields the $F$ given in Theorem 5 and concludes its proof.

Optimal demand curves with half-lines. Next we rewrite ( A-40) and express optimal demand curves in the two special cases when all agents learn the same additional information about $d$ in the form of either $d<\bar{d}$ or $d \geq \bar{d}$. On the 'lower half-line' when $\bar{d}_{1}=-\infty$ and $\bar{d}_{2}=\bar{d}$, i.e. the information set of agent $i$ is given by $\mathcal{L}_{i,-}=\left\{s_{i}, d-C u=l, d<\bar{d}\right\},(\mathrm{A}-40)$ simplifies to

$$
0=\gamma_{s} s_{i}+\gamma_{l} l-p-\alpha \sigma_{d \mid s, l}^{2} x_{i}-\sigma_{d \mid s, l} \frac{\phi\left(\frac{1}{\sigma_{d \mid s, l}}\left[\bar{d}-\left(\gamma_{s} s_{i}+\gamma_{l} l-\alpha \sigma_{d \mid s, l}^{2} x_{i}\right)\right]\right)}{\Phi\left(\frac{1}{\sigma_{d \mid s, l}}\left[\bar{d}-\left(\gamma_{s} s_{i}+\gamma_{l} l-\alpha \sigma_{d \mid s, l}^{2} x_{i}\right)\right]\right)} .
$$

After rearranging this equation, we obtain

$$
\frac{\bar{d}-p}{\sigma_{d \mid s, l}}=\Psi_{0}\left(\frac{1}{\sigma_{d \mid s, l}}\left[\bar{d}-\left(\gamma_{s} s_{i}+\gamma_{l} l-\alpha \sigma_{d \mid s, l}^{2} x_{i}\right)\right]\right),
$$

where $\Psi_{0}($.$) is the strictly increasing function defined in Lemma 5$, which is invertible as long as $\bar{d}-p$ is positive; we conjecture that this is the case, and verify later. Applying $\Psi_{0}^{-1}$ to both sides of (A-42) and rearranging, we obtain that agent $i$ 's optimal demand function under the information set $\mathcal{L}_{i,-}$ is given by

$$
x_{i,-}\left(s_{i}, p\right)=\frac{\gamma_{s} s_{i}+\gamma_{l} l-\bar{d}+\sigma_{d \mid s, l} \Psi_{0}^{-1}\left(\frac{\bar{d}-p}{\sigma_{d \mid s, l}}\right)}{\alpha \sigma_{d \mid s, l}^{2}} .
$$


Similarly, suppose now that all agents learn that $d$ is from an 'upper half-line', $\bar{d}_{1}=\bar{d}$ and $\bar{d}_{2}=\infty$, i.e. the information set of agent $i$ is given by $\mathcal{L}_{i,+}=\left\{s_{i}, d-C u=l, d \geq \bar{d}\right\}$. Equation (A-40) in this case becomes

$$
0=\gamma_{s} s_{i}+\gamma_{l} l-p-\alpha \sigma_{d \mid s, l}^{2} x_{i}+\sigma_{d \mid s, l} \frac{\phi\left(-\frac{1}{\sigma_{d \mid s, l}}\left[\bar{d}-\left(\gamma_{s} s_{i}+\gamma_{l} l-\alpha \sigma_{d \mid s, l}^{2} x_{i}\right)\right]\right)}{\Phi\left(-\frac{1}{\sigma_{d \mid s, l}}\left[\bar{d}-\left(\gamma_{s} s_{i}+\gamma_{l} l-\alpha \sigma_{d \mid s, l}^{2} x_{i}\right)\right]\right)},
$$

which is equivalent to

$$
-\frac{\bar{d}-p}{\sigma_{d \mid s, l}}=\Psi_{0}\left(-\frac{1}{\sigma_{d \mid s, l}}\left[\bar{d}-\left(\gamma_{s} s_{i}+\gamma_{l} l-\alpha \sigma_{d \mid s, l}^{2} x_{i}\right)\right]\right) .
$$

Applying $\Psi_{0}^{-1}$ to both sides of (A-44) and rearranging, we obtain that agent $i$ 's optimal demand function under the information set $\mathcal{L}_{i,+}$ is given by

$$
x_{i,+}\left(s_{i}, p\right)=\frac{\gamma_{s} s_{i}+\gamma_{l} l-\bar{d}-\sigma_{d \mid s, l} \Psi_{0}^{-1}\left(-\frac{\bar{d}-p}{\sigma_{d \mid s, l}}\right)}{\alpha \sigma_{d \mid s, l}^{2}} .
$$

Market clearing and equilibrium prices. Note that the optimal demand curves (A-43) and (A-45) depend linearly on the private signals $s_{i}$, and that the argument of $\Psi_{0}^{-1}$ is common across all agents, as we assumed they all share the same belief about the truncation $d<\bar{d}$ or $d \geq \bar{d}$. The exact law of large numbers hence implies that aggregating individual demands works the same way as in the linear case. Moreover, it illustrates that our example would also work in a version of the model with a finite number of agents; in that case both the usual linear and our discontinuous equilibrium price function reveals a noisy version of the average private signal of investors instead of a noisy version of the payoff $d$; see, e.g., Hellwig (1980).

In particular, suppose we are on a lower half-line. Using the optimal demand curves (A-43), the market-clearing condition (16) becomes

$u=\int x_{i,-}\left(s_{i}, p\right) \mathrm{d} i=\int \frac{\gamma_{s} s_{i}+\gamma_{l} l-\bar{d}+\sigma_{d \mid s, l} \Psi_{0}^{-1}\left(\frac{\bar{d}-p}{\sigma_{d \mid s, l}}\right)}{\alpha \sigma_{d \mid s, l}^{2}} \mathrm{~d} i=\frac{\gamma_{s} d+\gamma_{l} l-\bar{d}+\sigma_{d \mid s, l} \Psi_{0}^{-1}\left(\frac{\bar{d}-p}{\sigma_{d \mid s, l}}\right)}{\alpha \sigma_{d \mid s, l}^{2}}$.

Rearranging, applying $\Psi_{0}$ to both sides of the equation, then further rearranging, we obtain the equilibrium price function

$$
P_{-}(l)=\bar{d}(l)-\sigma_{d \mid s, l} \Psi_{0}\left(\frac{\bar{d}(l)-\left(\gamma_{s}+\gamma_{l}\right) l}{\sigma_{d \mid s, l}}\right) .
$$

Note that we needed $\bar{d}-p>0$ for (A-42) to be invertible, but as $\Psi_{0}(x)>0$ for all $x \in \mathbb{R}$, prices on lower half-lines are always below the cut $\bar{d}$.

Similarly, suppose we are on an upper half-line. From the optimal demand curves (A-45), (16) becomes

$$
u=\int x_{i,+}\left(s_{i}, p\right) \mathrm{d} i=\frac{\gamma_{s} d+\gamma_{l} l-\bar{d}-\sigma_{d \mid s, l} \Psi_{0}^{-1}\left(-\frac{\bar{d}-p}{\sigma_{d \mid s, l}}\right)}{\alpha \sigma_{d \mid s, l}^{2}} .
$$


Rearranging, we obtain the equilibrium price function

$$
P_{+}(l)=\bar{d}(l)+\sigma_{d \mid s, l} \Psi_{0}\left(-\frac{\bar{d}(l)-\left(\gamma_{s}+\gamma_{l}\right) l}{\sigma_{d \mid s, l}}\right) .
$$

We needed $\bar{d}-p<0$ for (A-44) to be invertible, but as $\Psi_{0}(x)>0$ for all $x \in \mathbb{R}$, prices on upper half-lines are always above the cut $\bar{d}$.

Equilibrium with a linear cut. Finally, we want to choose $\bar{d}$ such that the prices obtained in (A-46) and (A-47) correspond to a valid price function, i.e., it is a bijection between the price values and the level sets of $P$.

We claim that a vertical linear cut of the plane $\bar{d}(l)=J$ into two halves works well for any $J \in \mathbb{R}$. With detailed calculations following those of the GS case, we can show that these are the only linear cuts that provide valid equilibrium price functions. However, for brevity, we omit these calculations.

Substituting $\bar{d}(l)=J$ and $F=\gamma_{s}+\gamma_{l}$ into (A-46) and (A-47) we obtain

$$
P_{-}(l)=J-\sigma_{d \mid s, l} \Psi_{0}\left(\frac{J-F l}{\sigma_{d \mid s, l}}\right) \text { and } P_{+}(l)=J+\sigma_{d \mid s, l} \Psi_{0}\left(-\frac{J-F l}{\sigma_{d \mid s, l}}\right),
$$

which is the same as (17) due to $\chi=\sigma_{d \mid s, l}$. To show that these provide a valid price function

$$
P(d, u)= \begin{cases}P_{-}(d-G u) & \text { if } d<J \\ P_{+}(d-G u) & \text { if } d \geq J\end{cases}
$$

we need to show that $P_{-}$and $P_{+}$are both invertible functions, and that their images are disjoint.

Differentiating $P_{-}$and $P_{+}$in (A-48), we get

$$
P_{-}^{\prime}(l)=F \Psi_{0}^{\prime}\left(\frac{J-F l}{\chi}\right) \text { and } P_{+}^{\prime}(l)=F \Psi_{0}^{\prime}\left(-\frac{J-F l}{\chi}\right) .
$$

But Lemma 5 implies $\Psi_{0}^{\prime}(x)>0$ for all $x \in \mathbb{R}$, therefore, $P_{-}(l)$ and $P_{+}(l)$ are both increasing and thus invertible functions. Moreover, as $\Psi_{0}(x)>0$ for all $x \in \mathbb{R}, P_{-}(l)<J<P_{+}(l)$ for all $x \in \mathbb{R}$ and hence their images are disjoint. Finally, using Lemma 5 we also have

$$
\begin{aligned}
& \lim _{l \rightarrow \infty} P_{-}(l)=J-\chi \lim _{l \rightarrow \infty} \Psi_{0}\left(\frac{J-F l}{\chi}\right)=J-\chi \lim _{x \rightarrow-\infty} \Psi_{0}(x)=J \text { and } \\
& \lim _{l \rightarrow-\infty} P_{+}(l)=J+\chi \lim _{l \rightarrow-\infty} \Psi_{0}\left(-\frac{J-F l}{\chi}\right)=J+\chi \lim _{x \rightarrow-\infty} \Psi_{0}(x)=J .
\end{aligned}
$$

We conclude that the equilibrium price as a function of $l$ qualitatively looks the same as our leading example for a discontinuous equilibrium price function of the GS model. This concludes the proof of Theorem 6 . 


\section{Online Appendix to "Multiple Equilibria in Noisy Rational Expectations Economies"}

This online appendix contains the proofs of lemmas stated in Appendix A, useful closed forms for certain integral expressions, and the proofs of Propositions 1-5. For tractability, we restate the original lemmas, too. The functions $\phi($.$) and \Phi($.$) denote the pdf and cdf of the$ standard normal distribution, respectvely.

\section{Appendix OA-1 Useful function properties}

Lemma 4. The function

$$
\Psi_{-1}(x)=\frac{\phi(x)}{\Phi(x)}
$$

has the following three sets of properties:

(i) $\Psi_{-1}(x)>0 \forall x \in \mathbb{R}$, and its limits are $\lim _{x \rightarrow-\infty} \Psi_{-1}(x)=\infty$ and $\lim _{x \rightarrow \infty} \Psi_{-1}(x)=0$.

(ii) $\Psi_{-1}(x)$ is decreasing; its slope satisfies $-1<\Psi_{-1}^{\prime}(x)<0$ for all $x \in \mathbb{R}$ with limits $\lim _{x \rightarrow-\infty} \Psi_{-1}^{\prime}(x)=-1$ and $\lim _{x \rightarrow \infty} \Psi_{-1}^{\prime}(x)=0$, and $\Psi_{-1}^{\prime}$ increases monotonically in between.

(iii) $\Psi_{-1}(x)$ is convex: $\Psi_{-1}^{\prime \prime}(x)>0$ for all $x \in \mathbb{R}$.

Proof. $\Psi_{-1}$ is closely related to the so-called inverse Mills ratio or hazard function of the standard normal distribution, widely used in econometric analysis with truncated distributions (see, e.g., Greene (2003)). In particular, the inverse Mills ratio is given by $\phi(x) /[1-\Phi(x)]=$ $\Psi_{-1}(-x)$, and thus some parts of (i)-(iii) follow directly from certain properties of the inverse Mills ratio. We nevertheless provide some proofs that are useful for later parts of the appendix.

Throughout the proof we make repeated use of the fact that the first derivatives of $\phi(x)$ and $\phi^{2}(x)$ are given by

$$
\phi^{\prime}(x)=-x \phi(x) \text { and }\left(\phi^{2}(x)\right)^{\prime}=-2 x \phi^{2}(x) .
$$

(i) The first part of the statement holds because $\phi(x), \Phi(x)>0$ for all $x \in \mathbb{R}$. The third part of the statement follows from $\lim _{x \rightarrow \infty} \phi(x)=0$ and $\lim _{x \rightarrow \infty} \Phi(x)=1$. Regarding the middle part of the statement, $\lim _{x \rightarrow-\infty} \phi(x)=\lim _{x \rightarrow-\infty} \Phi(x)=0$, hence we use l'Hôpital's rule to obtain

$$
\lim _{x \rightarrow-\infty} \frac{\phi(x)}{\Phi(x)}=\lim _{x \rightarrow-\infty} \frac{\phi^{\prime}(x)}{\Phi^{\prime}(x)}=\lim _{x \rightarrow-\infty} \frac{-x \phi(x)}{\phi(x)}=\lim _{x \rightarrow-\infty}(-x)=\infty .
$$

(ii) First, differentiating $\Psi_{-1}$ we have

$$
\Psi_{-1}^{\prime}(x)=-\frac{\phi(x)[\phi(x)+x \Phi(x)]}{\Phi^{2}(x)}=-\Psi_{-1}(x)\left[x+\Psi_{-1}(x)\right] .
$$

We start with the limits: The second limit result of (ii) follows from $\lim _{x \rightarrow \infty} \Phi(x)=1$, $\lim _{x \rightarrow \infty} \phi(x)=0$, and $\lim _{x \rightarrow \infty} x \phi(x)=0$. On the other hand, when $x \rightarrow-\infty$, both the 
numerator and the denominator of (OA-2) converge to zero, hence with the repeated use of l'Hôpital's rule we obtain

$$
\begin{aligned}
& \lim _{x \rightarrow-\infty} \frac{\phi(x)[\phi(x)+x \Phi(x)]}{\Phi^{2}(x)}=\lim _{x \rightarrow-\infty} \frac{-x[\phi(x)+x \Phi(x)]+\Phi(x)}{2 \Phi(x)}=\lim _{x \rightarrow-\infty} \frac{-x \Phi(x)}{\phi(x)} \\
& =\lim _{x \rightarrow-\infty} \frac{-x \phi(x)-\Phi(x)}{-x \phi(x)}=\lim _{x \rightarrow-\infty} \frac{\left(x^{2}-2\right) \phi(x)}{\left(x^{2}-1\right) \phi(x)}=\lim _{x \rightarrow-\infty} \frac{x^{2}-2}{x^{2}-1}=1,
\end{aligned}
$$

Finally, $-1<\Psi_{-1}^{\prime}(x)<0 \forall x \in \mathbb{R}$ will follow from the combination of the limits and the convexity of $\Psi_{-1}$ : then $\Psi_{-1}^{\prime}$ is monotone increasing on $\mathbb{R}$, hence it is bounded from above and below by its values at $x \rightarrow \pm \infty$.

(iii) Convexity of $\Psi_{-1}(x)$ is equivalent to the convexity of $\Psi_{-1}(-x)=\phi(x) /[1-\Phi(x)]$, which is the inverse Mills ratio of the standard normal distribution. Since its convexity is proven in Sampford (1953), we omit it here.

Lemma 5. Suppose $\rho \geq 0$ constant. The function

$$
\Psi_{\rho}(x)=(1+\rho) x+\frac{\phi(x)}{\Phi(x)}
$$

has the following properties:

(i) If $\rho=0, \Psi_{0}(x)>0$ for all $x \in \mathbb{R}$, and its limits are $\lim _{x \rightarrow-\infty} \Psi_{0}(x)=0$ and $\lim _{x \rightarrow \infty} \Psi_{0}(x)=\infty$. If $\rho>0, \Psi_{\rho}(x)$ takes every real value and its limits are $\lim _{x \rightarrow-\infty} \Psi_{\rho}(x)=$ $-\infty$ and $\lim _{x \rightarrow \infty} \Psi_{\rho}(x)=\infty$.

(ii) $\Psi_{\rho}(x)$ is increasing; its slope satisfies $\rho<\Psi_{\rho}^{\prime}(x)<1+\rho$ for all $x \in \mathbb{R}$ with limits $\lim _{x \rightarrow-\infty} \Psi_{\rho}^{\prime}(x)=\rho$ and $\lim _{x \rightarrow \infty} \Psi_{\rho}^{\prime}(x)=1+\rho$, and $\Psi_{\rho}^{\prime}$ increases monotonically in between.

(iii) $\Psi_{\rho}(x)$ is convex: $\Psi_{\rho}^{\prime \prime}(x)>0$ for all $x \in \mathbb{R}$.

Proof. We first prove everything for the case $\rho=0$.

(i) First, notice that $\Psi_{0}(x)=x+\Psi_{-1}(x)=\frac{\phi(x)+x \Phi(x)}{\Phi(x)}$. It can be shown that

$$
\lim _{x \rightarrow-\infty}[\phi(x)+x \Phi(x)]=0 \text { and } \lim _{x \rightarrow \infty}[\phi(x)+x \Phi(x)]=\infty,
$$

moreover,

$$
\frac{\mathrm{d}}{\mathrm{d} x}[\phi(x)+x \Phi(x)]=\Phi(x)>0
$$

hence $\phi(x)+x \Phi(x)$ is positive and diverges to infinity. This implies $\Psi_{0}(x)>0$ and the limit when $x \rightarrow \infty$. For the other limit, $\lim _{x \rightarrow-\infty} \Phi(x)=\lim _{x \rightarrow-\infty}[\phi(x)+x \Phi(x)]=0$, so using l'Hoŝsital's rule twice we obtain

$$
\lim _{x \rightarrow-\infty} \frac{\phi(x)+x \Phi(x)}{\Phi(x)}=\lim _{x \rightarrow-\infty} \frac{\Phi(x)}{\phi(x)}=\lim _{x \rightarrow-\infty} \frac{\phi(x)}{-x \phi(x)}=\lim _{x \rightarrow-\infty}\left(-\frac{1}{x}\right)=0 .
$$

(ii)-(iii) Differentiating $\Psi_{0}$ we obtain $\Psi_{0}^{\prime}(x)=1+\Psi_{-1}^{\prime}(x)$ and $\Psi_{0}^{\prime \prime}(x)=\Psi_{-1}^{\prime \prime}(x)$, so the statements on the limits of $\Psi_{0}^{\prime}$ and the convexity of $\Psi_{0}$ follow from (ii) and (iii) of Lemma 4 , respectively. 
Finally, the rest of the results for $\rho>0$ follow from changing the function values by $\rho x$, which increases the slope everywhere by $\rho$ while not affecting convexity.

Lemma 6. Suppose $\rho \geq 0$ and $M>0$ constants. The function

$$
\Omega_{\rho}(x)=(1+\rho) x+\frac{\phi(x+M)-\phi(x-M)}{\Phi(x+M)-\Phi(x-M)}
$$

has the following properties:

(i) If $\rho=0, \Omega_{0}$ has limits $\lim _{x \rightarrow-\infty} \Omega_{0}(x)=-M$ and $\lim _{x \rightarrow \infty} \Omega_{0}(x)=-M$. If $\rho>0$, $\Omega_{\rho}(x)$ takes every real value and its limits are $\lim _{x \rightarrow-\infty} \Omega_{\rho}(x)=-\infty$ and $\lim _{x \rightarrow \infty} \Omega_{\rho}(x)=\infty$.

(ii) $\Omega_{\rho}(x)$ is always increasing, and its slope satisfies $\rho<\Omega_{\rho}^{\prime}(x)<\rho+M^{2}$ for all $x \in \mathbb{R}$.

Proof. We first prove everything for the case $\rho=0$.

(i) We start by writing

$$
\begin{aligned}
& \lim _{x \rightarrow \infty} \Omega_{0}(x)=\lim _{x \rightarrow \infty} \frac{x[\Phi(x+M)-\Phi(x-M)]+\phi(x+M)-\phi(x-M)}{\Phi(x+M)-\Phi(x-M)} \\
& =\lim _{x \rightarrow \infty} \frac{\Phi(x+M)-\Phi(x-M)-M \phi(x+M)-M(x-M) \phi(x-M)}{\phi(x+M)-\phi(x-M)} \\
& \quad=\lim _{x \rightarrow \infty} \frac{\Phi(x+M)-\Phi(x-M)}{\phi(x+M)-\phi(x-M)}-M \lim _{x \rightarrow \infty} \frac{\phi(x+M)+\phi(x-M)}{\phi(x+M)-\phi(x-M)},
\end{aligned}
$$

where the first equality is simple algebra, in the second equality we use l'Hôpital's rule because both the numerator and the denominator converge to zero and then simplify the numerator, and the third equality holds as long as the two limits are not both $\infty$ or both $-\infty$ at the same time, which we confirm shortly. Starting with the first term, we write

$$
\begin{aligned}
\lim _{x \rightarrow \infty} \frac{\Phi(x+M)-\Phi(x-M)}{\phi(x+M)-\phi(x-M)}=\lim _{x \rightarrow \infty} \frac{\phi(x+M)-\phi(x-M)}{-(x+M) \phi(x+M)+(x-M) \phi(x-M)} \\
=\lim _{x \rightarrow \infty} \frac{\exp \{-2 M x\}-1}{-(x+M) \exp \{-2 M x\}+(x-M)}=\lim _{x \rightarrow \infty} \frac{-1}{(x-M)}=0,
\end{aligned}
$$

where in the first step we use l'Hospital's rule, then we divide each term by $\phi(x-M)$ and use

$$
\frac{\phi(x+M)}{\phi(x-M)}=\exp \left\{\frac{(x-M)^{2}}{2}-\frac{(x+M)^{2}}{2}\right\}=\exp \{-2 M x\},
$$

finally we apply $\lim _{x \rightarrow \infty} \exp \{-2 M x\}=\lim _{x \rightarrow \infty} x \exp \{-2 M x\}=0$ because $M>0$. On the other hand, we have

$$
\lim _{x \rightarrow \infty} \frac{\phi(x+M)+\phi(x-M)}{\phi(x+M)-\phi(x-M)}=\lim _{x \rightarrow \infty} \frac{\exp \{-2 M x\}+1}{\exp \{-2 M x\}-1}=\lim _{x \rightarrow \infty} \frac{1}{-1}=-1,
$$

where in the first step we divide each term by $\phi(x-M)$ and use (OA-5), in the second step we use $\lim _{x \rightarrow \infty} \exp \{-2 M x\}=0$. Therefore, $\lim _{x \rightarrow \infty} \Omega_{0}(x)=M$. Finally, it is easy to confirm that $\Omega_{0}$ is an odd function, $\Omega_{0}(-x)=-\Omega_{0}(x)$, which implies $\lim _{x \rightarrow-\infty} \Omega_{0}(x)=-M$. 
(ii) Differentiating $\Omega_{0}(x)$ we obtain

$$
\Omega_{0}^{\prime}(x)=1-\frac{(x+M) \phi(x+M)-(x-M) \phi(x-M)}{\Phi(x+M)-\Phi(x-M)}-\left(\frac{\phi(x+M)-\phi(x-M)}{\Phi(x+M)-\Phi(x-M)}\right)^{2},
$$

but it is well-known that the expression on the RHS is the variance of a truncated standard normal distribution, i.e. $\Omega_{0}^{\prime}(x)=\operatorname{Var}[Y \mid x-M<Y<x+M]$ where $Y \sim N(0,1)$. Since the variance of a random variable is always positive, $\Omega_{0}$ must be an increasing function, $\Omega_{0}^{\prime}(x)>0$.

For the other side of the inequality, we rewrite (OA-6) as

$$
\Omega_{0}^{\prime}(x)=1-\frac{(x+M) \phi(x+M)-(x-M) \phi(x-M)}{\Phi(x+M)-\Phi(x-M)}+x^{2}+2 x \frac{\phi(x+M)-\phi(x-M)}{\Phi(x+M)-\Phi(x-M)}-\Omega_{0}^{2}(x) .
$$

The last term is $\Omega_{0}^{2}(x)$, and hence $\geq 0$ always. On the other hand, using $\phi^{\prime}(x)=-x \phi(x)$ and $\frac{\mathrm{d}(x \phi(x))}{\mathrm{d} x}=\left(1-x^{2}\right) \phi(x)$, we can write

$$
\begin{gathered}
\Phi(x+M)-\Phi(x-M)=\int_{x-M}^{x+M} \phi(z) \mathrm{d} z, \phi(x+M)-\phi(x-M)=\int_{x-M}^{x+M}(-z) \phi(z) \mathrm{d} z, \text { and } \\
(x+M) \phi(x+M)-(x-M) \phi(x-M)=\int_{x-M}^{x+M}\left(1-z^{2}\right) \phi(z) \mathrm{d} z .
\end{gathered}
$$

Using these identities and collecting terms, the non- $\Omega_{0}^{2}(x)$ terms of (OA-7) simplify to

$$
\begin{aligned}
1- & \frac{(x+M) \phi(x+M)-(x-M) \phi(x-M)}{\Phi(x+M)-\Phi(x-M)}+x^{2}+2 x \frac{\phi(x+M)-\phi(x-M)}{\Phi(x+M)-\Phi(x-M)}= \\
& =\frac{\int_{x-M}^{x+M} \phi(z) \mathrm{d} z-\int_{x-M}^{x+M}\left(1-z^{2}\right) \phi(z) \mathrm{d} z+x^{2} \int_{x-M}^{x+M} \phi(z) \mathrm{d} z+2 x \int_{x-M}^{x+M}(-z) \phi(z) \mathrm{d} z}{\Phi(x+M)-\Phi(x-M)} \\
= & \frac{\int_{x-M}^{x+M}\left[1-\left(1-z^{2}\right)+x^{2}-2 x z\right] \phi(z) \mathrm{d} z}{\Phi(x+M)-\Phi(x-M)}=\frac{\int_{x-M}^{x+M}(z-x)^{2} \phi(z) \mathrm{d} z}{\Phi(x+M)-\Phi(x-M)} .
\end{aligned}
$$

But for all $x-M<z<x+M$ we have $(z-x)^{2}<M$, therefore

$$
\frac{\int_{x-M}^{x+M}(z-x)^{2} \phi(z) \mathrm{d} z}{\Phi(x+M)-\Phi(x-M)}<\frac{M^{2} \int_{x-M}^{x+M} \phi(z) \mathrm{d} z}{\Phi(x+M)-\Phi(x-M)}=M^{2} .
$$

From here, (OA-7) yields $\Omega_{0}^{\prime}(x)<M^{2}-\Omega_{0}^{2}(x)<M^{2}$.

Finally, the results for $\rho>0$ follow from changing the function values by $\rho x$, which diverges when $x \rightarrow \pm \infty$, and increasing the slope everywhere by $\rho$.

Lemma 7. The function

$$
\digamma(x)=-\left[\Psi_{\rho}^{-1}(x)+\Psi_{\rho}^{-1}(-x)\right]
$$

is positive, convex, and even, it reaches its minimum at zero, and diverges to $\infty$ for $x \rightarrow \pm \infty$. 
Proof. It is imminent from the definition that $\digamma(x)$ is an even function, i.e. $\digamma(x)=\digamma(-x)$. Moreover, $\Psi_{\rho}(x)$ is convex from Lemma 5 , therefore $\Psi_{\rho}^{-1}$ is concave, and $\digamma$ is also convex. Since $\digamma$ is both symmetric with respect to the vertical axis and convex, it reaches its minimum at zero. Let us denote the unique root of $\Psi_{\rho}$ by $\bar{x}$, i.e. for which $\Psi_{\rho}(\bar{x})=0$; it is easy to see from the definition of $\Psi_{\rho}$ that $\bar{x}<0$. Therefore, the minimum of $\digamma$ is $\digamma(0)=-2 \Psi_{\rho}^{-1}(0)=-2 \bar{x}>0$. Finally, symmetry and convexity, together with the fact that $\digamma$ 's domain is the whole $\mathbb{R}$, imply that $\digamma$ is increasing on $\mathbb{R}^{+}$and hence diverges: $\lim _{x \rightarrow \infty} \digamma(x)=\infty$. Symmetry then also implies $\lim _{x \rightarrow-\infty} \digamma(x)=\infty$.

\section{Appendix OA-2 Useful integrals}

Lemma 8. If $X, Y, x, y \in \mathbb{R}$ are constants, we have

$$
\int_{-\infty}^{\infty} X \phi(X(t-x)) \Phi(Y(t-y)) \mathrm{d} t=\Phi\left(\frac{X Y}{\sqrt{X^{2}+Y^{2}}}(x-y)\right) .
$$

Proof. We write the LHS of (OA-9) as

$$
\begin{aligned}
\int_{-\infty}^{\infty} & X \phi(X(t-x)) \Phi(Y(t-y)) \mathrm{d} t=\int_{-\infty}^{\infty} X \phi(X(t-x)) \int_{-\infty}^{0} Y \phi(Y(w+t-y)) \mathrm{d} w \mathrm{~d} t \\
= & \int_{-\infty}^{0} \int_{-\infty}^{\infty} X \phi(X(t-x)) Y \phi(Y(t-(y-w))) \mathrm{d} t \mathrm{~d} w \\
= & \int_{-\infty}^{0} X Y \phi\left(\frac{X Y}{\sqrt{X^{2}+Y^{2}}}[w-(y-x)]\right) \int_{-\infty}^{\infty} \phi\left(\sqrt{X^{2}+Y^{2}}\left[t-\frac{X^{2} x+Y^{2}(y-w)}{X^{2}+Y^{2}}\right]\right) \mathrm{d} t \mathrm{~d} w \\
= & \int_{-\infty}^{0} \frac{X Y}{\sqrt{X^{2}+Y^{2}}} \phi\left(\frac{X Y}{\sqrt{X^{2}+Y^{2}}}[w-(y-x)]\right) \mathrm{d} w=\Phi\left(\frac{X Y}{\sqrt{X^{2}+Y^{2}}}(x-y)\right),
\end{aligned}
$$

where the first equality is due to $\Phi(z)=\int_{-\infty}^{0} \phi(Y(w+z)) \mathrm{d} w$, the second uses Fubini to change the order of integration, the third collects the $t$ terms, the fourth recognizes that the inner integral equals $1 / \sqrt{X^{2}+Y^{2}}$, and the fifth performs the integral. This confirms (OA-9).

Lemma 9. If $X, Y, x, y \in \mathbb{R}$ are constants, we have

$$
\begin{aligned}
& \int_{-\infty}^{\infty} t X \phi(X(t-x)) \Phi(Y(t-y)) \mathrm{d} t \\
& \quad=x \Phi\left(\frac{X Y}{\sqrt{X^{2}+Y^{2}}}(x-y)\right)+\frac{1}{X^{2}} \frac{X Y}{\sqrt{X^{2}+Y^{2}}} \phi\left(\frac{X Y}{\sqrt{X^{2}+Y^{2}}}(x-y)\right) .
\end{aligned}
$$


Proof. We first rewrite the LHS of (OA-10) as

$$
\begin{aligned}
& \int_{-\infty}^{\infty} t X \phi(X(t-x)) \Phi(Y(t-y)) \mathrm{d} t=x \int_{-\infty}^{\infty} X \phi(X(t-x)) \Phi(Y(t-y)) \mathrm{d} t \\
& -\frac{1}{X} \int_{-\infty}^{\infty}\left[-X^{2}(t-x)\right] \phi(X(t-x)) \Phi(Y(t-y)) \mathrm{d} t .
\end{aligned}
$$

Observing the second term of the RHS, we can write

$$
\begin{aligned}
\int_{-\infty}^{\infty} \overbrace{\left[-X^{2}(t-x)\right] \phi(X(t-x))}^{f^{\prime}} \overbrace{\Phi(Y(t-y))}^{g} \mathrm{~d} t \\
\quad=[\phi(X(t-x)) \Phi(Y(t-y))]_{-\infty}^{\infty}-\int_{-\infty}^{\infty} \overbrace{\phi(X(t-x))}^{f} \overbrace{Y \phi(Y(t-y))}^{g^{\prime}} \mathrm{d} t \\
=-Y \int_{-\infty}^{\infty} \phi(X(t-x)) \phi(Y(t-y)) \mathrm{d} t \\
=-\frac{Y}{\sqrt{X^{2}+Y^{2}}} \phi\left(\frac{X Y}{\sqrt{X^{2}+Y^{2}}}(x-y)\right) \int_{-\infty}^{\infty} \sqrt{X^{2}+Y^{2}} \phi\left(\sqrt{X^{2}+Y^{2}}\left(t-\frac{X^{2} x+Y^{2} y}{X^{2}+Y^{2}}\right)\right) \mathrm{d} t \\
=-\frac{Y}{\sqrt{X^{2}+Y^{2}}} \phi\left(\frac{X Y}{\sqrt{X^{2}+Y^{2}}}(x-y)\right) .
\end{aligned}
$$

where the first equality is due to integration by parts, the second uses that $\Phi(Y(t-y))$ is bounded and $\lim _{t \rightarrow \pm \infty} \phi(X(t-x))=0$ hence the $[.]_{-\infty}^{\infty}$ term is zero, the third collects all $t$ terms, and the fourth recognizes that the remaining integral equals 1 . Returning to (OA-11), we thus have

$$
\begin{aligned}
\int_{-\infty}^{\infty} & t X \phi(X(t-x)) \Phi(Y(t-y)) \mathrm{d} t \\
= & x \int_{-\infty}^{\infty} X \phi(X(t-x)) \Phi(Y(t-y)) \mathrm{d} t+\frac{1}{X} \frac{Y}{\sqrt{X^{2}+Y^{2}}} \phi\left(\frac{X Y}{\sqrt{X^{2}+Y^{2}}}(x-y)\right) \\
= & x \Phi\left(\frac{X Y}{\sqrt{X^{2}+Y^{2}}}(x-y)\right)+\frac{1}{X^{2}} \frac{X Y}{\sqrt{X^{2}+Y^{2}}} \phi\left(\frac{X Y}{\sqrt{X^{2}+Y^{2}}}(x-y)\right)
\end{aligned}
$$

where the second equality uses (OA-9). This yields (OA-10).

Lemma 10. If $X, Y, x, y \in \mathbb{R}$ are constants, we have

$$
\begin{gathered}
\int_{-\infty}^{\infty} t^{2} X \phi(X(t-x)) \Phi(Y(t-y)) \mathrm{d} t=\left(x^{2}+\frac{1}{X^{2}}\right) \Phi\left(\frac{X Y}{\sqrt{X^{2}+Y^{2}}}(x-y)\right) \\
+\left(x+\frac{X^{2} x+Y^{2} y}{X^{2}+Y^{2}}\right) \frac{1}{X^{2}} \frac{X Y}{\sqrt{X^{2}+Y^{2}}} \phi\left(\frac{X Y}{\sqrt{X^{2}+Y^{2}}}(x-y)\right) .
\end{gathered}
$$


Proof. We start by decomposing the LHS of (OA-12) as

$$
\begin{aligned}
& \int_{-\infty}^{\infty} t^{2} X \phi(X(t-x)) \Phi(Y(t-y)) \mathrm{d} t=x \int_{-\infty}^{\infty} t X \phi(X(t-x)) \Phi(Y(t-y)) \mathrm{d} t \\
& \quad+\int_{-\infty}^{\infty}(t-x) t X \phi(X(t-x)) \Phi(Y(t-y)) \mathrm{d} t .
\end{aligned}
$$

We first rewrite the second term of the RHS as

$$
\begin{aligned}
& \int_{-\infty}^{\infty}(t-x) t X \phi(X(t-x)) \Phi(Y(t-y)) \mathrm{d} t \\
& =-\frac{1}{X} \int_{-\infty}^{\infty} \overbrace{\left[-X^{2}(t-x)\right] \phi(X(t-x))}^{f^{\prime}} \overbrace{t \Phi(Y(t-y))}^{g} \mathrm{~d} t \\
& =-\frac{1}{X}[[\phi(X(t-x)) t \Phi(Y(t-y))]_{-\infty}^{\infty}-\int_{-\infty}^{\infty} \overbrace{\phi(X(t-x))}^{f} \overbrace{[\Phi(Y(t-y))+Y t \phi(Y(t-y))}^{f} \mathrm{~d} t] \\
& =\frac{1}{X^{2}} \int_{-\infty}^{\infty} X \phi(X(t-x)) \Phi(Y(t-y)) \mathrm{d} t+\frac{Y}{X} \int_{-\infty}^{\infty} t \phi(X(t-x)) \phi(Y(t-y)) \mathrm{d} t \\
& =\frac{1}{X^{2}} \Phi\left(\frac{X Y}{\sqrt{X^{2}+Y^{2}}}(x-y)\right)+\frac{Y}{X} \int_{-\infty}^{\infty} t \phi(X(t-x)) \phi(Y(t-y)) \mathrm{d} t \\
& =\frac{1}{X^{2}} \Phi\left(\frac{X Y}{\sqrt{X^{2}+Y^{2}}}(x-y)\right)+\frac{Y}{X} \frac{1}{\sqrt{X^{2}+Y^{2}}} \phi\left(\frac{X Y}{\sqrt{X^{2}+Y^{2}}}(x-y)\right) \times \\
& \times \int_{-\infty}^{\infty} t \sqrt{X^{2}+Y^{2}} \phi\left(\sqrt{X^{2}+Y^{2}}\left(t-\frac{X^{2} x+Y^{2} y}{X^{2}+Y^{2}}\right)\right) \mathrm{d} t \\
& =\frac{1}{X^{2}} \Phi\left(\frac{X Y}{\sqrt{X^{2}+Y^{2}}}(x-y)\right)+\frac{1}{X^{2}} \frac{X Y}{\sqrt{X^{2}+Y^{2}}} \frac{X^{2} x+Y^{2} y}{X^{2}+Y^{2}} \phi\left(\frac{X Y}{\sqrt{X^{2}+Y^{2}}}(x-y)\right),
\end{aligned}
$$

where the second equality is due to integration by parts, the third uses that the $[.]_{-\infty}^{\infty}$ term is zero and decomposes the integral, the fourth uses (OA-9), the fifth collects the $t$ terms in the integral and brings the rest outside the integral, and the sixth recognizes that the integral is the expected value of a normally distributed random variable with mean $\frac{X^{2} x+Y^{2} y}{X^{2}+Y^{2}}$ and thus 
is equal to this term. On the other hand, the first term of (OA-13) follows from (OA-10). Substituting these into (OA-13), we obtain

$$
\begin{aligned}
& \int_{-\infty}^{\infty} t^{2} X \phi(X(t-x)) \Phi(Y(t-y)) \mathrm{d} t \\
& \quad=x \Phi\left(\frac{X Y}{\sqrt{X^{2}+Y^{2}}}(x-y)\right)\left[x+\frac{1}{X^{2}} \frac{X Y}{\sqrt{X^{2}+Y^{2}}} \frac{\phi\left(\frac{X Y}{\sqrt{X^{2}+Y^{2}}}(x-y)\right)}{\Phi\left(\frac{X Y}{\sqrt{X^{2}+Y^{2}}}(x-y)\right)}\right] \\
& \quad+\frac{1}{X^{2}} \Phi\left(\frac{X Y}{\sqrt{X^{2}+Y^{2}}}(x-y)\right)+\frac{1}{X^{2}} \frac{X Y}{\sqrt{X^{2}+Y^{2}}} \frac{X^{2} x+Y^{2} y}{X^{2}+Y^{2}} \phi\left(\frac{X Y}{\sqrt{X^{2}+Y^{2}}}(x-y)\right),
\end{aligned}
$$

which is equivalent to (OA-12).

Lemma 11. If $X, Y, x, y \in \mathbb{R}$ are constants, we have

$$
\begin{aligned}
& \int_{-\infty}^{\infty} t^{3} X \phi(X(t-x)) \Phi(Y(t-y)) \mathrm{d} t=x\left(x^{2}+\frac{3}{X^{2}}\right) \Phi\left(\frac{X Y}{\sqrt{X^{2}+Y^{2}}}(x-y)\right) \quad(\text { OA- } 14) \\
& +\frac{1}{X^{2}}\left[x\left(x+\frac{X^{2} x+Y^{2} y}{X^{2}+Y^{2}}\right)+\left(\frac{X^{2} x+Y^{2} y}{X^{2}+Y^{2}}\right)^{2}+\frac{2}{X^{2}}+\frac{1}{X^{2}+Y^{2}}\right] \frac{X Y}{\sqrt{X^{2}+Y^{2}}} \phi\left(\frac{X Y}{\sqrt{X^{2}+Y^{2}}}(x-y)\right) .
\end{aligned}
$$

Proof. We write

$$
\begin{aligned}
& \int_{-\infty}^{\infty} t^{3} X \phi(X(t-x)) \Phi(Y(t-y)) \mathrm{d} t=x \int_{-\infty}^{\infty} t^{2} X \phi(X(t-x)) \Phi(Y(t-y)) \mathrm{d} t \\
& \quad+\int_{-\infty}^{\infty} t^{2}(t-x) X \phi(X(t-x)) \Phi(Y(t-y)) \mathrm{d} t
\end{aligned}
$$

where the first term, because of (OA-12), becomes

$$
\begin{gathered}
x \int_{-\infty}^{\infty} t^{2} X \phi(X(t-x)) \Phi(Y(t-y)) \mathrm{d} t=x\left(x^{2}+\frac{1}{X^{2}}\right) \Phi\left(\frac{X Y}{\sqrt{X^{2}+Y^{2}}}(x-y)\right) \\
+x\left(x+\frac{X^{2} x+Y^{2} y}{X^{2}+Y^{2}}\right) \frac{1}{X^{2}} \frac{X Y}{\sqrt{X^{2}+Y^{2}}} \phi\left(\frac{X Y}{\sqrt{X^{2}+Y^{2}}}(x-y)\right) .
\end{gathered}
$$


The second term can be written as

$$
\begin{aligned}
& \int_{-\infty}^{\infty} t^{2}(t-x) X \phi(X(t-x)) \Phi(Y(t-y)) \mathrm{d} t \\
& =-\frac{1}{X} \int_{-\infty}^{\infty} \overbrace{\left[-X^{2}(t-x)\right] \phi(X(t-x))}^{t^{\prime} \Phi(Y(t-y))} \mathrm{d} t \\
& =-\frac{1}{X}\left[\phi(X(t-x)) t^{2} \Phi(Y(t-y))\right]_{-\infty}^{\infty}-\int_{-\infty}^{\infty} \overbrace{\phi(X(t-x))}^{f^{\prime}} \overbrace{\left[2 t \Phi(Y(t-y))+Y t^{2} \phi(Y(t-y))\right]}^{f} \mathrm{~d} t] \\
& =\frac{1}{X} \int_{-\infty}^{\infty} \phi(X(t-x))\left[2 t \Phi(Y(t-y))+Y t^{2} \phi(Y(t-y))\right] \mathrm{d} t \\
& =\frac{1}{X}\left[2 \int_{-\infty}^{\infty} t \phi(X(t-x)) \Phi(Y(t-y)) \mathrm{d} t+Y \int_{-\infty}^{\infty} t^{2} \phi(X(t-x)) \phi(Y(t-y)) \mathrm{d} t\right] .
\end{aligned}
$$

Equation (OA-10) implies that the first term inside the bracket is

$$
\begin{gathered}
2 \int_{-\infty}^{\infty} t \phi(X(t-x)) \Phi(Y(t-y)) \mathrm{d} t=\frac{2}{X} \int_{-\infty}^{\infty} t X \phi(X(t-x)) \Phi(Y(t-y)) \mathrm{d} t \\
=\frac{2}{X} \Phi\left(\frac{X Y}{\sqrt{X^{2}+Y^{2}}}(x-y)\right)\left[x+\frac{1}{X^{2}} \frac{X Y}{\sqrt{X^{2}+Y^{2}}} \frac{\phi\left(\frac{X Y}{\sqrt{X^{2}+Y^{2}}}(x-y)\right)}{\Phi\left(\frac{X Y}{\sqrt{X^{2}+Y^{2}}}(x-y)\right)}\right],
\end{gathered}
$$

on the other hand

$$
\begin{aligned}
& \int_{-\infty}^{\infty} t^{2} \phi(X(t-x)) \phi(Y(t-y)) \mathrm{d} t \\
& =\frac{1}{\sqrt{X^{2}+Y^{2}}} \phi\left(\frac{X Y}{\sqrt{X^{2}+Y^{2}}}(x-y)\right) \int_{-\infty}^{\infty} \sqrt{X^{2}+Y^{2}} t^{2} \phi\left(\sqrt{X^{2}+Y^{2}}\left(t-\frac{X^{2} x+Y^{2} y}{X^{2}+Y^{2}}\right)\right) \mathrm{d} t \\
& =\frac{1}{\sqrt{X^{2}+Y^{2}}} \phi\left(\frac{X Y}{\sqrt{X^{2}+Y^{2}}}(x-y)\right)\left[\left(\frac{X^{2} x+Y^{2} y}{X^{2}+Y^{2}}\right)^{2}+\frac{1}{X^{2}+Y^{2}}\right],
\end{aligned}
$$

because the integral is simply $E\left[t^{2}\right]=E^{2}[t]+\operatorname{Var}[t]$, where $t \sim N\left(\frac{X^{2} x+Y^{2} y}{X^{2}+Y^{2}}, \frac{1}{X^{2}+Y^{2}}\right)$. Combining these results, after some algebra we obtain (OA-14). 


\section{Appendix OA-3 Proofs of Propositions 1-5}

Proof of Proposition 1. From (13) and (12), after some algebra, we obtain that the difference between the price on the right and left side of $\bar{s}(l)$ is given by

$$
\Delta P(l)=P_{+}(l)-P_{-}(l)=-\frac{\theta}{\vartheta}\left[\Psi_{\rho}^{-1}(\lambda(B l-D))+\Psi_{\rho}^{-1}(\lambda(B l-D))\right]=\frac{\theta}{\vartheta} \digamma(\lambda(B l-D)),
$$

where $\digamma($.$) is defined in (\mathrm{OA}-8)$. The properties of $\Delta P($.$) then follow from Lemma 7$.

Proof of Propositions 2 and 5. In the GS equilibrium, expectation and variance conditional on the information content of the market price, $E[d \mid l]$ and $\operatorname{Var}[d \mid l]$, are given by (10); the expectation is linear in $l$ and, due to the joint normality, conditional variance is constant. Since the market price $p$ is then linear in $l$, the expectation $E\left[d \mid P_{G S}=p\right]$ is linearly increasing in $p$, the variance $\operatorname{Var}\left[d \mid P_{G S}=l\right]$ is constant, and $S k e w\left[d \mid P_{G S}=p\right]=0$ due to joint normality.

In the LC equilbirum, the price reveals both $l$ and the halfline (either $s<\bar{s}(l)$ or $s \geq \bar{s}(l)$ ). Hence, for all $p<D$ we have $E\left[d \mid P_{L C}=p\right]=E[d \mid l, s<\bar{s}(l)]$ and for all $p>D$ we have $E\left[d \mid P_{L C}=p\right]=E[d \mid l, \geq \bar{s}(l)]$, and similarly for conditional variance and skewness.

Conditional expectation and its properties. To obtain $E[d \mid l, s<\bar{s}(l)]$ we first rewrite (A-3) for the case $s \in(-\infty, \bar{s})$ :

$$
f_{d \mid l, s<\bar{s}}(d \mid l, s<\bar{s})=\frac{1}{\sigma_{d \mid l}} \phi\left(\frac{1}{\sigma_{d \mid l}}\left(d-\beta_{l} l\right)\right) \frac{\Phi\left(-\frac{\sigma_{l \mid s}}{\sigma_{l \mid d} \sigma_{\varepsilon}}\left(d-\frac{\sigma_{l \mid d}^{2} \bar{s}-\sigma_{\varepsilon}^{2} l}{\sigma_{l \mid s}^{2}}\right)\right)}{\Phi\left(\frac{1}{\theta}\left(\bar{s}-\frac{\sigma_{s}^{2} l}{\sigma_{l}^{2} l}\right)\right)}
$$

and thus, introducing the notation $E\left[d \mid l_{-}\right]=E[d \mid l, s<\bar{s}]$, we have

$$
\begin{aligned}
E & {\left[d \mid l_{-}\right]=\int_{-\infty}^{\infty} d f_{d \mid l, s<\bar{s}}(d \mid l, s<\bar{s}) \mathrm{d} d } \\
& =\frac{1}{\Phi\left(\frac{1}{\theta}\left(\bar{s}-\frac{\sigma_{s}^{2}}{\sigma_{l}^{2}} l\right)\right)} \int_{-\infty}^{\infty} d \frac{1}{\sigma_{d \mid l}} \phi\left(\frac{1}{\sigma_{d \mid l}}\left(d-\beta_{l} l\right)\right) \Phi\left(-\frac{\sigma_{l \mid s}}{\sigma_{l \mid d} \sigma_{\varepsilon}}\left(d-\frac{\sigma_{l \mid d}^{2} \bar{s}-\sigma_{\varepsilon}^{2} l}{\sigma_{l \mid s}^{2}}\right)\right) \mathrm{d} d .
\end{aligned}
$$

To express the integral in closed form, we use Lemma 9 for

$$
X=\frac{1}{\sigma_{d \mid l}}, x=\beta_{l} l, Y=-\frac{\sigma_{l \mid s}}{\sigma_{l \mid d} \sigma_{\varepsilon}}, \text { and } y=\frac{\sigma_{l \mid d}^{2} \bar{s}-\sigma_{\varepsilon}^{2} l}{\sigma_{l \mid s}^{2}} .
$$

After rearranging the result, we obtain

$$
E\left[d \mid l_{-}\right]=\beta_{l} l-\frac{\sigma_{l \mid s} \sigma_{d}^{2}}{\sigma_{s} \sigma_{l}} \frac{\phi\left(\frac{1}{\theta}\left(\bar{s}-\frac{\sigma_{s}^{2}}{\sigma_{l}^{2}} l\right)\right)}{\Phi\left(\frac{1}{\theta}\left(\bar{s}-\frac{\sigma_{s}^{2}}{\sigma_{l}^{2}} l\right)\right)}=\beta_{l} l-\sqrt{\sigma_{d \mid l}^{2}-\sigma_{d \mid s}^{2}} \Psi_{-1}(\Lambda(l)),
$$


where we use the definition of $\Psi_{-1}$ and that $\sigma_{d \mid s}^{2} \leq \sigma_{d \mid l}^{2}$ due to $l=s-C u$ being a noisy information about $s$, and introduce the notation

$$
\Lambda(l)=\frac{1}{\theta}\left(\bar{s}(l)-\frac{\sigma_{s}^{2}}{\sigma_{l}^{2}} l\right) .
$$

Similarly, for the case $s \in(\bar{s}, \infty),(\mathrm{A}-3)$ becomes

$$
f_{d \mid l, s \geq \bar{s}}(d \mid l, s \geq \bar{s})=\frac{1}{\sigma_{d \mid l}} \phi\left(\frac{1}{\sigma_{d \mid l}}\left(d-\beta_{l} l\right)\right) \frac{\Phi\left(\frac{\sigma_{l \mid s}}{\sigma_{l \mid d} \sigma_{\varepsilon}}\left(d-\frac{\sigma_{l \mid d}^{2} \bar{s}-\sigma_{\varepsilon}^{2} l}{\sigma_{l \mid s}^{2}}\right)\right)}{\Phi\left(-\frac{1}{\theta}\left(\bar{s}-\frac{\sigma_{s}^{2}}{\sigma_{l}^{2} l}\right)\right)}
$$

and thus

$$
\begin{aligned}
& E\left[d \mid l_{+}\right]=E[d \mid l, s \geq \bar{s}]=\int_{-\infty}^{\infty} d f_{d \mid l, s \geq \bar{s}}(d \mid l, s \geq \bar{s}) \mathrm{d} d \\
& =\frac{1}{\Phi\left(-\frac{1}{\theta}\left(\bar{s}-\frac{\sigma_{s}^{2}}{\sigma_{l}^{2}} l\right)\right)} \int_{-\infty}^{\infty} d \frac{1}{\sigma_{d \mid l}} \phi\left(\frac{1}{\sigma_{d \mid l}}\left(d-\beta_{l} l\right)\right) \Phi\left(\frac{\sigma_{l \mid s}}{\sigma_{l \mid d} \sigma_{\varepsilon}}\left(d-\frac{\sigma_{l \mid d}^{2} \bar{s}-\sigma_{\varepsilon}^{2} l}{\sigma_{l \mid s}^{2}}\right)\right) \mathrm{d} d .
\end{aligned}
$$

To express the integral in closed form, we use Lemma 9 for

$$
X=\frac{1}{\sigma_{d \mid l}}, x=\beta_{l} l, Y=\frac{\sigma_{l \mid s}}{\sigma_{l \mid d} \sigma_{\varepsilon}}, \text { and } y=\frac{\sigma_{l \mid d}^{2} \bar{s}-\sigma_{\varepsilon}^{2} l}{\sigma_{l \mid s}^{2}} .
$$

After simplifying the result, we obtain

$$
E\left[d \mid l_{+}\right]=\beta_{l} l+\frac{\sigma_{l \mid s} \sigma_{d}^{2}}{\sigma_{s} \sigma_{l}} \frac{\phi\left(-\frac{1}{\theta}\left(\bar{s}-\frac{\sigma_{s}^{2}}{\left.\sigma_{l}^{2} l\right)}\right)\right.}{\Phi\left(-\frac{1}{\theta}\left(\bar{s}-\frac{\sigma_{s}^{2}}{\sigma_{l}^{2}} l\right)\right)}=\beta_{l} l+\sqrt{\sigma_{d \mid l}^{2}-\sigma_{d \mid s}^{2}} \Psi_{-1}(-\Lambda(l)) .
$$

We next confirm the properties of $E[d \mid p]$ when $p \in(D, \infty)$; the $p \in(-\infty, D)$ case follows analogously. For this, we start with studying the limits of $E\left[d \mid l_{+}\right]$when $l \rightarrow \pm \infty$. Notice that since the equilibrium $\bar{s}(l)$ is linear and decreasing in $l, \Lambda$ is also linearly decreasing and thus

$$
\lim _{l \rightarrow-\infty} \Lambda(l)=\infty \text { and } \lim _{l \rightarrow \infty} \Lambda(l)=-\infty .
$$

According to Lemma 4, we then have

$$
\lim _{l \rightarrow \infty} \Psi_{-1}(-\Lambda(l))=\lim _{x \rightarrow \infty} \Psi_{-1}(x)=0,
$$

and thus $E\left[d \mid l_{+}\right]$behaves like $\beta_{l} l$ when $l \rightarrow \infty$. On the other hand, after some algebra we can rewrite (OA-21) as

$$
E\left[d \mid l_{+}\right]=\beta_{s} \bar{s}+\sqrt{\sigma_{d \mid l}^{2}-\sigma_{d \mid s}^{2}} \Psi_{0}(-\Lambda(l)) .
$$

But according to Lemma 5,

$$
\lim _{l \rightarrow-\infty} \Psi_{0}(-\Lambda(l))=\lim _{x \rightarrow-\infty} \Psi_{0}(x)=0
$$


hence $\lim _{l \rightarrow-\infty} E\left[d \mid l_{+}\right]=\lim _{l \rightarrow-\infty} \beta_{s} \bar{s}(l)=\infty$. As $l \rightarrow \infty$ is equivalent to $p \rightarrow \infty$ and $l \rightarrow-\infty$ to $p \searrow D$, we have shown that for both extremes of $(D, \infty), E[d \mid p]$ goes to infinity.

Next we study the shape of $E[d \mid p]$ as a function of $p$. Since both $E\left[d \mid l_{+}\right]$and $P_{+}(l)$ are infinitely differentiable functions of $l$ on $(D, \infty)$, the slope of $E[d \mid p]$ satisfies

$$
\frac{\mathrm{d} E[d \mid p]}{\mathrm{d} p}=\frac{\frac{\mathrm{d} E\left[d \mid l_{+}\right]}{\mathrm{d} l}}{P_{+}^{\prime}(l)}
$$

Because $P_{+}$is an increasing function, the denominator of the RHS is positive, and the LHS has the same sign as the numerator of the RHS. From (OA-21) and Lemma 4 we know get that $E\left[d \mid l_{+}\right]$is convex in $l$, which, together with its limits, implies that there is a unique $l^{*} \in(D, \infty)$ for which $\left.\frac{\mathrm{d} E\left[d \mid l_{+}\right]}{\mathrm{d} l}\right|_{l=l^{*}}=0$, and the slope is negative for all $l<l^{*}$ and positive for all $l>l^{*}$. Therefore, we can conclude that $E[d \mid p]$ is decreasing for all $p \in\left(D, P_{+}\left(l^{*}\right)\right)$, reaches its minimum at $p=P_{+}\left(l^{*}\right)$, and increaseses (to infinity) when $p \in\left(P_{+}\left(l^{*}\right), \infty\right)$.

Conditional variance and its properties. For variances, we have

$$
\operatorname{Var}\left[d \mid l_{-}\right]=\operatorname{Var}[d \mid l, s<\bar{s}]=E\left[d^{2} \mid l_{-}\right]-E^{2}\left[d \mid l_{-}\right] .
$$

From (OA-15),

$$
\begin{aligned}
& E\left[d^{2} \mid l_{-}\right]=\int_{-\infty}^{\infty} d^{2} f_{d \mid l, s<\bar{s}}(d \mid l, s<\bar{s}) \mathrm{d} d \\
& \quad=\frac{1}{\Phi\left(\frac{1}{\theta}\left(\bar{s}-\frac{\sigma_{s}^{2}}{\sigma_{l}^{2}} l\right)\right)} \int_{-\infty}^{\infty} d^{2} \frac{1}{\sigma_{d \mid l}} \phi\left(\frac{1}{\sigma_{d \mid l}}\left(d-\beta_{l} l\right)\right) \Phi\left(-\frac{\sigma_{l \mid s}}{\sigma_{l \mid d} \sigma_{\varepsilon}}\left(d-\frac{\sigma_{l \mid d}^{2} \bar{s}-\sigma_{\varepsilon}^{2} l}{\sigma_{l \mid s}^{2}}\right)\right) \mathrm{d} d,
\end{aligned}
$$

and to express the integral in closed form, we can use Lemma 10 for (OA-16). Substituting it into (OA-25), using (OA-17) and (OA-18), and rearranging, we finally obtain

$\operatorname{Var}\left[d \mid l_{-}\right]=\sigma_{d \mid l}^{2}-\left(\sigma_{d \mid l}^{2}-\sigma_{d \mid s}^{2}\right) \Psi_{-1}(\Lambda(l))\left[\Lambda(l)+\Psi_{-1}(\Lambda(l))\right]=\sigma_{d \mid l}^{2}+\left(\sigma_{d \mid l}^{2}-\sigma_{d \mid s}^{2}\right) \Psi_{-1}^{\prime}(\Lambda(l))$,

where the second step recognizes the derivative of $\Psi_{-1}(x)$; see, e.g., (OA-2). Proceeding similarly for the $s \geq \bar{s}$ case, we get

$$
\operatorname{Var}\left[d \mid l_{+}\right]=\sigma_{d \mid l}^{2}+\left(\sigma_{d \mid l}^{2}-\sigma_{d \mid s}^{2}\right) \Psi_{-1}^{\prime}(-\Lambda(l)) .
$$

Here we consider $\lim _{p \rightarrow \infty} \operatorname{Var}[d \mid p]$ and $\lim _{p \searrow D} \operatorname{Var}[d \mid p]$; the other two limits when $p \rightarrow-\infty$ and $p \nearrow D$ follow similarly. First, (OA-22) and (OA-27) together imply

$$
\lim _{p \rightarrow \infty} \operatorname{Var}[d \mid p]=\lim _{l \rightarrow \infty} \operatorname{Var}\left[d \mid l_{+}\right]=\sigma_{d \mid l}^{2}+\left(\sigma_{d \mid l}^{2}-\sigma_{d \mid s}^{2}\right) \lim _{x \rightarrow \infty} \Psi_{-1}^{\prime}(x)=\sigma_{d \mid l}^{2},
$$

where in the last step we used (ii) of Lemma 4. Similarly, we write

$$
\lim _{p \searrow D} \operatorname{Var}[d \mid p]=\lim _{l \rightarrow-\infty} \operatorname{Var}\left[d \mid l_{+}\right]=\sigma_{d \mid l}^{2}+\left(\sigma_{d \mid l}^{2}-\sigma_{d \mid s}^{2}\right) \lim _{x \rightarrow-\infty} \Psi_{-1}^{\prime}(x)=\sigma_{d \mid s}^{2},
$$

where again the last step follows from (ii) of Lemma 4. 
Regarding the shape of the conditional variance, we show that it increases on $(D, \infty)$ : From (OA-22) we have that when $l$ increases, $-\Lambda(l)$ also increases. According to Lemma 4, $\Psi_{-1}$ is a convex function, therefore $\Psi_{-1}^{\prime}(-\Lambda(l))$ increases in $l$. (OA-27) then implies that $\operatorname{Var}\left[d \mid l_{+}\right]$also increases in $l$, and since $P_{+}(l)$ increases in $l, \operatorname{Var}[d \mid p]$ increases in $p$ on $(D, \infty)$. Analogously, we get that $\operatorname{Var}[d \mid p]$ is decreasing for $p \in(-\infty, D)$.

Conditional skewness and its properties. For the skewness of returns, we have to compute the third central moment:

$$
\operatorname{Skew}[d-p \mid p]=\operatorname{Skew}[d \mid p]=E\left[(d-E[d \mid p])^{3} \mid p\right]=E\left[d^{3} \mid p\right]-3 E[d \mid p] \operatorname{Var}[d \mid p]-E^{3}[d \mid p] .
$$

Because the distribution of $d \mid p$ when $s \in(-\infty, \bar{s})$ is given by (OA-15), to express the first term of the RHS, we use Lemma 11 for (OA-16) and, after some algebra, obtain

$$
E\left[d^{3} \mid l_{-}\right]=\left(\beta_{l} l\right)^{3}+3 \sigma_{d \mid l}^{2} \beta_{l} l-\left[\left(\beta_{l} l\right)^{2}+\beta_{l} \beta_{s} l \bar{s}+\left(\beta_{s} \bar{s}\right)^{2}+2 \sigma_{d \mid l}^{2}+\sigma_{d \mid s}^{2}\right] \sqrt{\sigma_{d \mid l}^{2}-\sigma_{d \mid s}^{2}} \Psi_{-1}(\Lambda(l)) .
$$

Substituting it into (OA-30), combining with (OA-17) and (OA-26) and rearranging, yields the following expression for skewness:

$\operatorname{Skew}\left[d-p \mid l_{-}\right]=-\left(\sigma_{d \mid l}^{2}-\sigma_{d \mid s}^{2}\right)^{3 / 2} \Psi_{-1}(\Lambda(l))\left\{\left[\Lambda(l)+\Psi_{-1}(\Lambda(l))\right]\left[\Lambda(l)+2 \Psi_{-1}(\Lambda(l))\right]-1\right\}$.

However, differentiating (A-1) twice we obtain

$$
\Psi_{-1}^{\prime \prime}(x)=\Psi_{-1}(x)\left\{\left[x+\Psi_{-1}(x)\right]\left[x+2 \Psi_{-1}(x)\right]-1\right\},
$$

therefore, (OA-31) simplifies to

$$
S k e w\left[d-p \mid l_{-}\right]=-\left(\sigma_{d \mid l}^{2}-\sigma_{d \mid s}^{2}\right)^{3 / 2} \Psi_{-1}^{\prime \prime}(\Lambda(l)) .
$$

Similarly, for the $l_{+}$case we use Lemma 11 for (OA-20), and obtain

$$
E\left[d^{3} \mid l_{+}\right]=\left(\beta_{l} l\right)^{3}+3 \sigma_{d \mid l}^{2} \beta_{l} l+\left[\left(\beta_{l} l\right)^{2}+\beta_{l} \beta_{s} l \bar{s}+\left(\beta_{s} \bar{s}\right)^{2}+2 \sigma_{d \mid l}^{2}+\sigma_{d \mid s}^{2}\right] \sqrt{\sigma_{d \mid l}^{2}-\sigma_{d \mid s}^{2}} \Psi_{-1}(-\Lambda(l)) .
$$

Substituting it into (OA-30), combining it with (OA-21) and (OA-27) and rearranging, yields that skewness is given by

$$
\text { Skew }\left[d-p \mid l_{+}\right]=\left(\sigma_{d \mid l}^{2}-\sigma_{d \mid s}^{2}\right)^{3 / 2} \Psi_{-1}^{\prime \prime}(-\Lambda(l)) .
$$

But part (iii) of Lemma 4 states that $\Psi_{-1}(x)$ is convex, i.e., $\Psi_{-1}^{\prime \prime}(x)>0$ for all $x \in \mathbb{R}$. Therefore, (OA-33) implies skewness is negative for all $p<D$ and (OA-34) implies skewness is positive for all $p>D$. This completes the proof of Propositions 2 and 5 .

Proof of Proposition 3. Formally, we show that $x_{U}(p)$ is downward sloping both on $(-\infty, D)$ and $(D, \infty)$, and has $\operatorname{limits} \lim _{p \rightarrow-\infty} x_{U}(p)=\lim _{p \searrow_{D}} x_{U}(p)=\infty$ and $\lim _{p} \nearrow_{D} x_{U}(p)=$ $\lim _{p \rightarrow \infty} x_{U}(p)=-\infty$. 
We look at the $p \in(D, \infty)$ case here. As in equilibrium uninformed demand $x_{U}$ satisfies (A-6) while the price on $(D, \infty)$ is given by $(13)$, we simply have

$$
\lim _{p \searrow D} x_{U}(p)=\lim _{l \rightarrow-\infty} \frac{P_{+}(l)-\beta_{s} l}{(1-\omega) \beta_{s} C}=\frac{D}{(1-\omega) \beta_{s} C}-\lim _{l \rightarrow-\infty} \frac{l}{(1-\omega) C}=\infty .
$$

On the other hand, from (A-23) we have

$$
\lim _{l \rightarrow \infty} P_{+}^{\prime}(l)=(1+\rho) B\left(1-\frac{\rho}{\lim _{l \rightarrow \infty} \Psi_{\rho}^{\prime}\left(\Psi_{\rho}^{-1}\left(\frac{1}{\theta}\left(\rho \kappa B l-\frac{\kappa \Theta}{\vartheta}\right)\right)\right)}\right)=B,
$$

where the second equality is due to part (ii) of Lemma 5 . But then because $B<\beta_{s}$, we must have $\lim _{l \rightarrow \infty}\left[P_{+}(l)-\beta_{s} l\right]=-\infty$, and hence

$$
\lim _{p \rightarrow \infty} x_{U}(p)=\lim _{l \rightarrow \infty} \frac{P_{+}(l)-\beta_{s} l}{(1-\omega) \beta_{s} C}=-\infty .
$$

Finally, we want to show that $x_{U}(p)$ is locally downward sloping everywhere on $(D, \infty)$. For this end, since both $x_{U}$ and $P_{+}$are differentiable functions, we can write the slope as

$$
\frac{\mathrm{d} x_{U}}{\mathrm{~d} p}=\frac{\frac{\mathrm{d} x_{U}}{\mathrm{~d} l}}{\frac{\mathrm{d} P_{+}}{\mathrm{d} l}}=\frac{1}{(1-\omega) \beta_{s} C}\left(1-\frac{\beta_{s}}{P_{+}^{\prime}(l)}\right)
$$

i.e. $\mathrm{d} x_{U} / \mathrm{d} p<0$ iff $P_{+}^{\prime}(l)<\beta_{s}$. Going back to (A-23), Lemma 5 implies $\Psi_{\rho}^{\prime}(x)<1+\rho$ for all $x \in \mathbb{R}$, so $1-\frac{\rho}{\Psi_{\rho}^{\prime}(x)}<\frac{1}{1+\rho}$ for all $x \in \mathbb{R}$, and, after some algebra, we obtain $P_{+}^{\prime}(l)<B<\beta_{s}$. Therefore, $x_{U}(p)$ is downward sloping on $(D, \infty)$, and has $\operatorname{limits} \lim _{p \searrow D} x_{U}(p)=\infty$ and $\lim _{p \rightarrow \infty} x_{U}(p)=-\infty$.

Analogously, we can show that $\lim _{p \rightarrow-\infty} x_{U}(p)=\infty$ and $\lim _{p} \nearrow_{D} x_{U}(p)=-\infty$, with $\mathrm{d} x_{U} / \mathrm{d} p<0$ for all $p \in(-\infty, D)$. But this means that there is a jump in uninformed demand at $p=D$, and hence it is globally not downward sloping.

Proof of Proposition 4. From the proof of Proposition 2 we obtain both $\lim _{p \searrow D} E[d-p \mid p]=$ $\lim _{p \searrow D} E[d \mid p]-D=\infty$ and $\lim _{p} \nearrow_{D} E[d-p \mid p]=\lim _{p}{ }_{D} E[d \mid p]-D=-\infty$. But as $P_{+}(l), P_{-}(l), E\left[d \mid l_{+}\right]$and $E\left[d \mid l_{-}\right]$are all continuous functions of $l, E[d-p \mid p]$ is continuous on both $(-\infty, D)$ and $(D, \infty)$. Continuity together with the limits for $p \nearrow D$ and $p \searrow D$ implies that around $p=D$ there is a jump in the expected return, and, unlike in the GS equilibrium, a higher price can imply a higher expected return. Formally, for any expected return level $\bar{r}$ there exists a price level $\bar{p}>D$ such that $E[d-p \mid p]>\bar{r}$ for all $p \in(D, \bar{p})$, and for any expected return level $\underline{r}$ there exists a price level $\underline{p}<D$ such that $E[d-p \mid p]<\underline{r}$ for all $p \in(\underline{p}, D)$.

Finally, although this is not required to the proposition, we can show that expected return $E[d-p \mid p]$ behaves similarly to that in the GS model when $p$ is either very high or very low $(p \rightarrow \pm \infty)$, in line with the bottom panel of Figure 3. Part (ii) of Lemma 4 and (OA-18) imply $\lim _{l \rightarrow \infty} \Psi_{-1}(-\Lambda(l))=0$, thus from $(\mathrm{OA}-21), \lim _{l \rightarrow \infty}\left(E\left[d \mid l_{+}\right]-\beta_{l} l\right)=0$. Combining it with (OA-35), we obtain

$$
\lim _{l \rightarrow \infty} \frac{\mathrm{d}\left(E\left[d \mid l_{+}\right]-P_{+}(l)\right)}{\mathrm{d} l}=\beta_{l}-B<0 .
$$

Therefore, $\lim _{p \rightarrow \infty} E[d-p \mid p]=\lim _{l \rightarrow \infty}\left(E\left[d \mid l_{+}\right]-P_{+}(l)\right)=-\infty$. Analogously, we can show that $\lim _{p \rightarrow-\infty} E[d-p \mid p]=\lim _{l \rightarrow-\infty}\left(E\left[d \mid l_{-}\right]-P_{-}(l)\right)=\infty$. 